\title{
Design, synthesis and biological evaluation of new peptide- based ureas and thioureas as potential antagonists of the thrombin receptor PAR1
}

Pilar Ventosa-Andrés, ${ }^{a}$ Ángel M. Valdivielso, ${ }^{a}$ Ioannis Pappos, ${ }^{b}$ M. Teresa García-López, ${ }^{a}$ Nikos E. Tsopanoglou ${ }^{b}$ and Rosario Herranz ${ }^{a}$.

${ }^{a}$ Instituto de Química Médica (CSIC), Juan de la Cierva 3, 28006 Madrid, Spain.

${ }^{b}$ Department of Pharmacology, Medical School, University of Patras, 26500 Rio-Patras, Greece.

\begin{abstract}
By applying a diversity oriented synthesis strategy for the search of new antagonists of the thrombin receptor PAR1, a series of peptide-based ureas and thioureas, including analogues of the PAR1 reference antagonist RWJ-58259, has been designed and synthesized. The general synthetic scheme involves reduction of basic amino acid-derived amino nitriles by hydrogen transfer from hydrazine monohydrate in the presence of Raney $\mathrm{Ni}$, followed by reaction with diverse isocyanates and isothiocyanates, and protecting group removal. All new compounds have been evaluated as inhibitors of human platelet aggregation induced by the PAR1 agonist SFLLRN. Some protected peptide-based ureas displayed significant antagonist activity.
\end{abstract}

Keywords: PAR1 antagonists; Peptide-derived ureas; Peptide-derived thioureas; $\alpha$-Amino nitriles; Platelet antiaggregant activity.

\section{Introduction}

In addition to the key role of thrombin in the blood coagulation cascade [1], this serine protease regulates multiple effects on an increasing variety of cells, such as: platelets $[2,3]$, endothelial and smooth muscle cells [2, 4], neurons and astrocytes in the nervous system [2, 4-7], immune and inflammatory cells [8, 9], osteoblasts [10], and tumor cells [11-14]. These cellular effects are mainly mediated by the activation of the protease-activated receptor PAR1 [15]. This is a G-protein coupled receptor (GPCR) that is activated by the thrombin-catalyzed cleavage of the N-terminal extracellular domain at the $\mathrm{Arg}^{41} / \mathrm{Ser}^{42}$ peptide bond, which

"Corresponding autor: Fax: +34 915644853; Tel: +34 912587537; E-mail: rosario@iqm.csic.es 
unveils the recognition sequence SFLLRN that acts as a tethered activation ligand. The conformational changes induced by activation favors the coupling of PAR1 with heterotrimeric G-proteins, which consequently activate complex cellular signaling cascades $[16,17]$.

Since PAR1 is mainly expressed in platelets, where its activation induces aggregation, it has been proposed that PAR1 antagonists could be good antithrombotic agents without the hemorrhagic drawbacks of thrombin inhibitors. Based on this suggestion, up to now, PAR1 antagonists have been searched almost exclusively in relation to the cardiovascular system [3, 18, 19]. However, numerous studies have shown that PAR1 is overexpressed in invasive and metastatic tumors and that its expression levels directly correlate with the degree of invasiveness of the cancer [20-28]. Based on these facts, this receptor is starting to be also considered a promising target for cancer therapy [15], particularly in the search of angiogenesis inhibitors [29].

The first potent PAR1 antagonists were SFLLRN-based peptidomimetic ureas, represented by the optimized antagonist RWJ-58259 (Figure 1) [19]. This antagonist showed protection against thrombus formation in nonhuman primates, providing the first in vivo proof for supporting the potential clinical utility of PAR1 antagonists, although, its low oral bioavailability stopped its clinical development [19]. Later, several laboratories have reported a few series of antagonists obtained from HTS of diverse libraries of non-peptide small molecules, followed by optimization [19, 30]. The most advanced of these antagonists is SCH-530348 (named vorapaxar, Figure 1), derived from the natural product himbacine, which currently is undergoing Phase III clinical trials in patients with acute coronary syndrome and in patients with atherosclerosis [31-33]. Up to now, there is not structural information on the binding sites of these PAR1 antagonists to be used for structure-based design of new antagonists. However, recent mutagenesis studies on thrombin and/or PAR1 [34-42], X-ray of thrombin crystallized with diverse N-terminal fragments of PAR1 [43, 44], and NMR studies on the (Ala ${ }^{26}-\mathrm{Hse}^{103}$ ) N-terminal sequence of PAR1 [45] have shown that the first thrombin/PAR1 interaction is produced between the exosite I of thrombin and the hirudin-like sequence of PAR1 $\left(\mathrm{K}^{51} \mathrm{YEPF}^{55}\right)$, and that this first interaction is essential and determinant for high affinity. It seems that the hydrophobic residues F34, I82, L65 and Y76, 


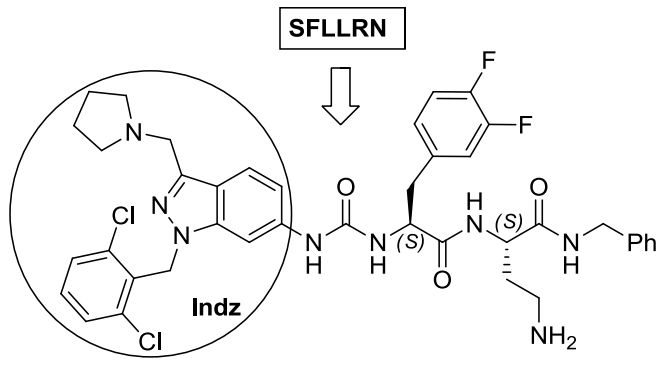

(RWJ-58259)

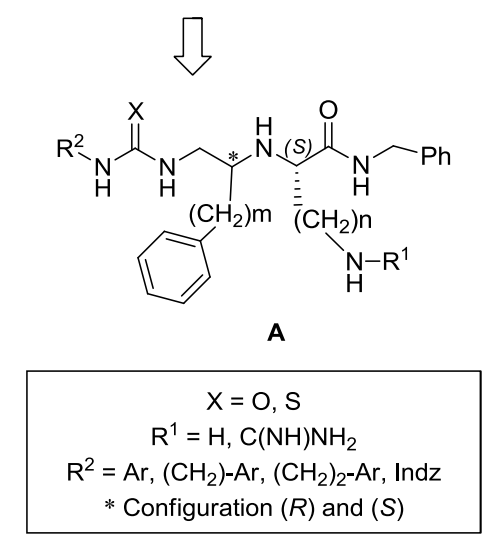

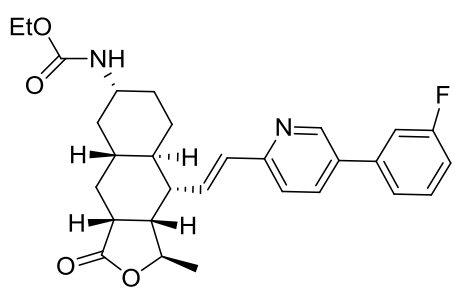

$(\mathrm{SCH}-530348)$

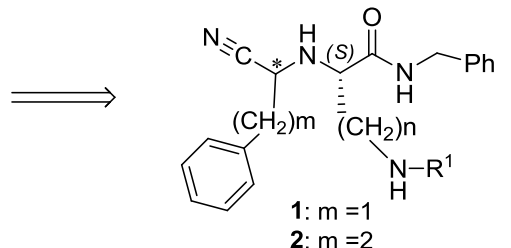

a: $n=3, R^{1}=B o c$

b: $n=4, R^{1}=B o c$

c: $n=3, \mathrm{R}^{1}=\mathrm{C}\left(\mathrm{NH}_{2}\right) \mathrm{N}-\mathrm{Pbf}$

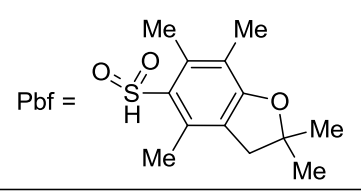

Fig. 1. Selected PAR1 antagonists and proposed new structures

and the basic residues R67 and R73, of the exosite I of thrombin are important for high affinity. Taking into account this knowledge, we started a project directed to the search of new PAR1 antagonists based on these hot spots of the exosite I of thrombin for PAR1. As these hot spots are discontinuous and are not localized in a defined secondary structure, we decided to use a diversity oriented synthesis (DOS) strategy for the search of peptidomimetics. For this purpose, we planned the synthesis of diverse small directed libraries of different scaffolds able to display, at least, one or two aromatic groups and one or two basic groups at variable distances and orientations. Among these structures, our first objective was the synthesis of ureas and thioureas of general formula $\mathbf{A}$, which could be considered as analogues of the reference antagonist RWJ-58259. To this aim, we devised a synthetic scheme using basic amino acid derived $\alpha$-amino nitriles $\mathbf{1}$ and $\mathbf{2}$ (Figure 1) as key diversity generation intermediates [46]. Herein, we report the synthesis of ureas and thioureas $\mathbf{A}$ and their evaluation as human PAR1 antagonists in a platelet aggregation assay. 


\section{Results and discussion}

\subsection{Synthesis}

The proposed general synthetic scheme for the preparation of ureas and thioureas $\mathbf{A}$ involves cyano-reduction of the protected $\alpha$-amino nitriles $\mathbf{1}$ and $\mathbf{2}$, followed by reaction of the resulting primary amines with isocyanates or isothiocyanates and subsequent removal of protecting groups. The starting basic amino acid-derived $\alpha$-amino nitriles $\mathbf{1}$ and $\mathbf{2}$ were obtained by a modified Strecker reaction as (1:1) epimeric mixtures at the cyano-supporting stereocenter, except for the arginine derivatives 1c, which were obtained in an $(R) /(S)$ ratio of (1:3) [47]. Only the epimeric mixtures $(\boldsymbol{R S})-\mathbf{1 a}, \mathbf{b}$ could be chromatographically resolved into the respective $(R)$ - and $(S)$-epimers. Therefore, $(\boldsymbol{R S})-\mathbf{1 c}$ and $(\boldsymbol{R S})-\mathbf{2 a}, \mathbf{b}$ were used as such epimeric mixtures through the synthetic pathway.

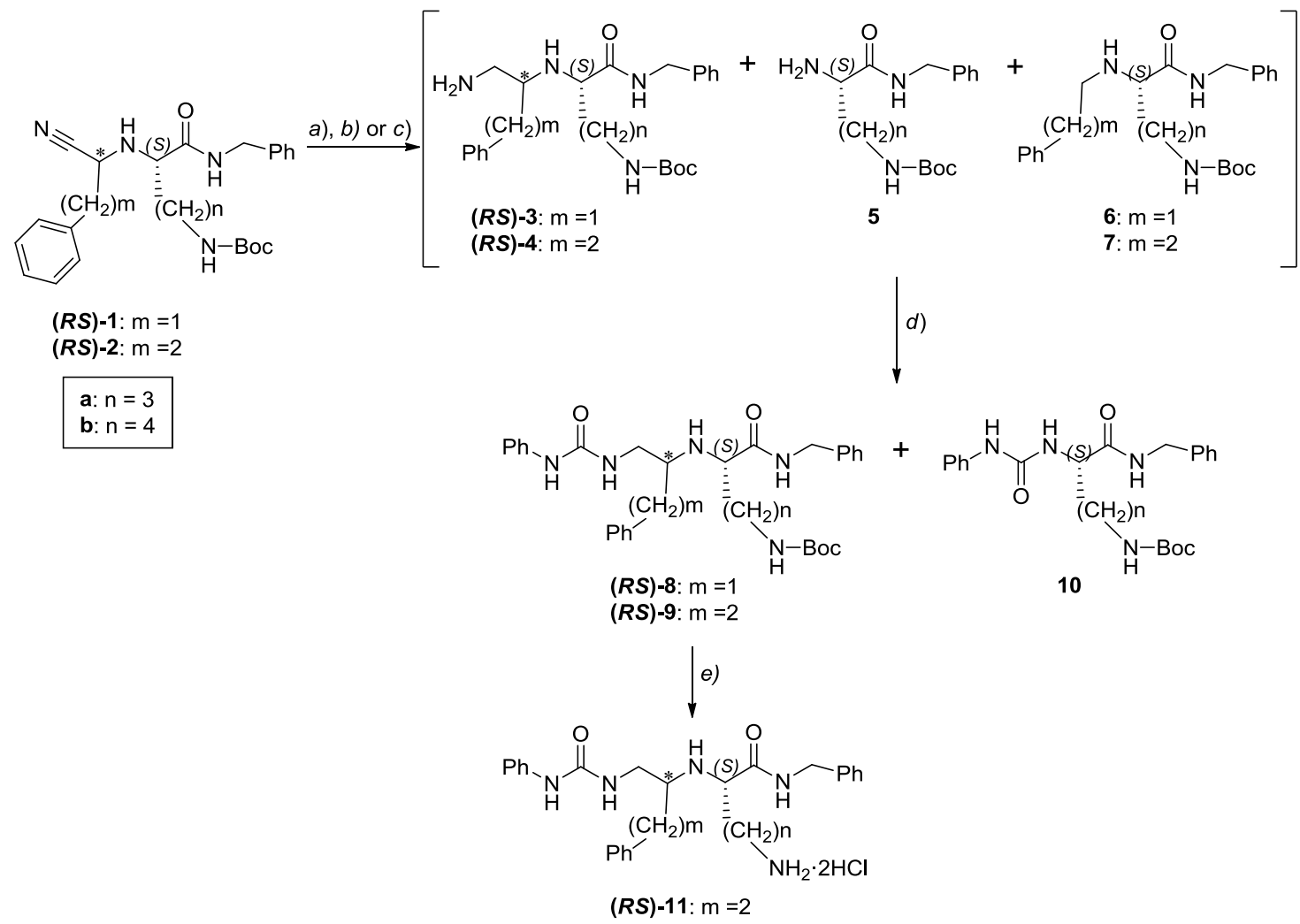

Scheme 1. Reagents and conditions: (a) $\mathrm{H}_{2}, \mathrm{Pd}(\mathrm{C}), \mathrm{MeOH}$, rt. (b) $\mathrm{H}_{2}$, Raney Ni, $\mathrm{MeOH}$, rt. (c) $\mathrm{NH}_{2} \mathrm{NH}_{2} \cdot \mathrm{H}_{2} \mathrm{O}$, Raney $\mathrm{Ni}, \mathrm{MeOH}$ or $\mathrm{EtOH}, \mathrm{T}$ and time as indicated in Table 1. (d) $\mathrm{PhNCO}, \mathrm{CH}_{2} \mathrm{Cl}_{2}, 0^{\circ} \mathrm{C}$. (e) $3 \mathrm{M} \mathrm{HCl}, \mathrm{EtOAc}, \mathrm{rt}$.

The epimeric mixture (RS)-1a was used as a model for optimizing the cyano reduction. Initially, this was attempted by $10 \% \operatorname{Pd}(\mathrm{C})$ or Raney Ni catalyzed hydrogenation. However, as 
shown in Scheme 1 and Table 1, the HPLC MS analysis of the crude reaction showed that the expected primary amines $(\boldsymbol{R S})$-3a were obtained along with variable percentages of 5a and 6a, resulting from retro-Strecker reaction and reduction of the intermediate imine after removal of HCN, respectively. The difficulties in the purification of $(\boldsymbol{R S})$-3a from the crude reaction mixtures moved us to try the reduction by hydrogen transfer, using hydrazine monohydrate as hydrogen source and Raney $\mathrm{Ni}$ as catalyst [48]. After the study of reaction conditions (Table 1), the formation of the side product $\mathbf{6 a}$ could be completely avoided, but not that of the retro-Strecker reaction 5a. This side reaction was minimized to a $5 \%$ by carrying out the reduction under refluxing $\mathrm{MeOH}$ for $5 \mathrm{~min}$, using 20 equivalents of hydrazine monohydrate and $100 \mathrm{mg} / \mathrm{mmol}$ of Raney Ni (entry 7). These optimized conditions were applied to the reduction of the other starting $\alpha$-amino nitriles. It is interesting to point out the importance of the Raney Ni activation, as well as the efficiency of the stirring, to make the reduction as faster as possible, in order to minimize the presence of the amino nitrile within the reaction medium, for minimizing the retro-Strecker reaction. Besides, in the phenylacetaldehyde derivatives $\mathbf{3}(\mathrm{m}=1)$, the reduction of the $(R)$-epimer was slower than that of the corresponding $(S)$-epimer and, consequently, its percentage of degradation via retroStrecker was significantly higher [see for example different yields for reduction of $(R)$ - and $(S)$ epimers of $\mathbf{1 a}$ and $\mathbf{1 b}$ in Table 2]. In the case of the Arg derivative $\mathbf{3 c}$, the difference in reactivity between epimers made that the minor $(R)$-epimer were lost in the reduction.

\section{Table 1}

Optimization of the reduction conditions for $(\boldsymbol{R S})-\mathbf{1 a}$

\begin{tabular}{|c|c|c|c|c|c|c|c|}
\hline \multirow{2}{*}{ Entry } & \multirow{2}{*}{ Catalyst } & \multirow{2}{*}{$\mathrm{H}_{2}$ source } & \multirow{2}{*}{$\mathrm{T}\left({ }^{\circ} \mathrm{C}\right)$} & \multirow{2}{*}{$\mathrm{t}(\min )$} & \multicolumn{3}{|c|}{ Yield $(\%)^{a}$} \\
\hline & & & & & $(\boldsymbol{R S})-\mathbf{3 a}$ & $5 \mathbf{a}$ & $\mathbf{6 a}$ \\
\hline 1 & $10 \% \operatorname{Pd}(\mathrm{C})$ & $\mathrm{H}_{2}$ & $\mathrm{rt}$ & $24 \mathrm{~h}$ & 80 & 8 & 12 \\
\hline 2 & Raney Ni (100 mg/mmol) & $\mathrm{H}_{2}$ & $\mathrm{rt}$ & $24 \mathrm{~h}$ & 54 & 6 & 40 \\
\hline 3 & Raney Ni (100 mg/mmol) & $\mathrm{NH}_{2} \mathrm{NH}_{2} \cdot \mathrm{H}_{2} \mathrm{O}$ & 85 & 30 & 37 & 63 & - \\
\hline 4 & Raney Ni (100 mg/mmol) & $\mathrm{NH}_{2} \mathrm{NH}_{2} \cdot \mathrm{H}_{2} \mathrm{O}$ & $\mathrm{rt}$ & 30 & 67 & 33 & - \\
\hline 5 & Raney Ni (200 mg/mmol) & $\mathrm{NH}_{2} \mathrm{NH}_{2} \cdot \mathrm{H}_{2} \mathrm{O}$ & $\mathrm{rt}$ & 30 & 73 & 27 & - \\
\hline 6 & Raney Ni (100 mg/mmol) & $\mathrm{NH}_{2} \mathrm{NH}_{2} \cdot \mathrm{H}_{2} \mathrm{O}$ & $\mathrm{rt}$ & 90 & 92 & 8 & - \\
\hline 7 & Raney Ni (100 mg/mmol) & $\mathrm{NH}_{2} \mathrm{NH}_{2} \cdot \mathrm{H}_{2} \mathrm{O}$ & 65 & 5 & 95 & 5 & - \\
\hline
\end{tabular}

${ }^{a}$ Determined by HPLC-MS analysis. 
Due to the difficulties found in the separation of the primary amines $\mathbf{3}$ and $\mathbf{4}$ from the side product of the retro-Strecker reaction $\mathbf{5}$, the crude reduction mixtures were initially used without purification for the synthesis of the desired ureas $\mathbf{8}$ and $\mathbf{9}$. However, the difficulties in the purification of these ureas remained, and several column chromatographies were required for their separation from the ureas $\mathbf{1 0}$, byproducts derived from the amino amides $\mathbf{5}$, with considerable loss in the yield. Only the epimeric mixture of ornithine-derived ureas $(\boldsymbol{R S})-\mathbf{9 a}$ could be resolved during this chromatographic purification. In view of these results, to avoid the purification difficulties, we decided to isolate the reduction intermediate amines as the corresponding Fmoc-derivatives. This strategy was studied in the phenylacetaldehyde derivatives $(\boldsymbol{R})$ - and $(\boldsymbol{S})-\mathbf{3 a}, \mathbf{b}$ and $(\boldsymbol{R S})-\mathbf{3 c}$. At this point, it is important to note that when the Fmoc-protection was carried out under the standard basic conditions, using $\mathrm{Na}_{2} \mathrm{CO}_{3}$ as $\mathrm{HCl}$ acceptor in dioxane/water [49], almost complete epimerization of one of the two chiral centers was observed. This epimerization was completely avoided by using propylene oxide as $\mathrm{HCl}$ acceptor in $\mathrm{CH}_{2} \mathrm{Cl}_{2}$, to obtain the Fmoc-protected amines $(\boldsymbol{R})$ - and $(\boldsymbol{S})-\mathbf{1 2 a}, \mathbf{b}$ and $(S)-12 \mathrm{c}$ in 30-72\% overall yield from the amino nitriles $(\boldsymbol{R})$ - and $(\boldsymbol{S})-\mathbf{3 a}, \mathbf{b}$ and $(\boldsymbol{R S})-\mathbf{3 c}$ (Scheme 2).

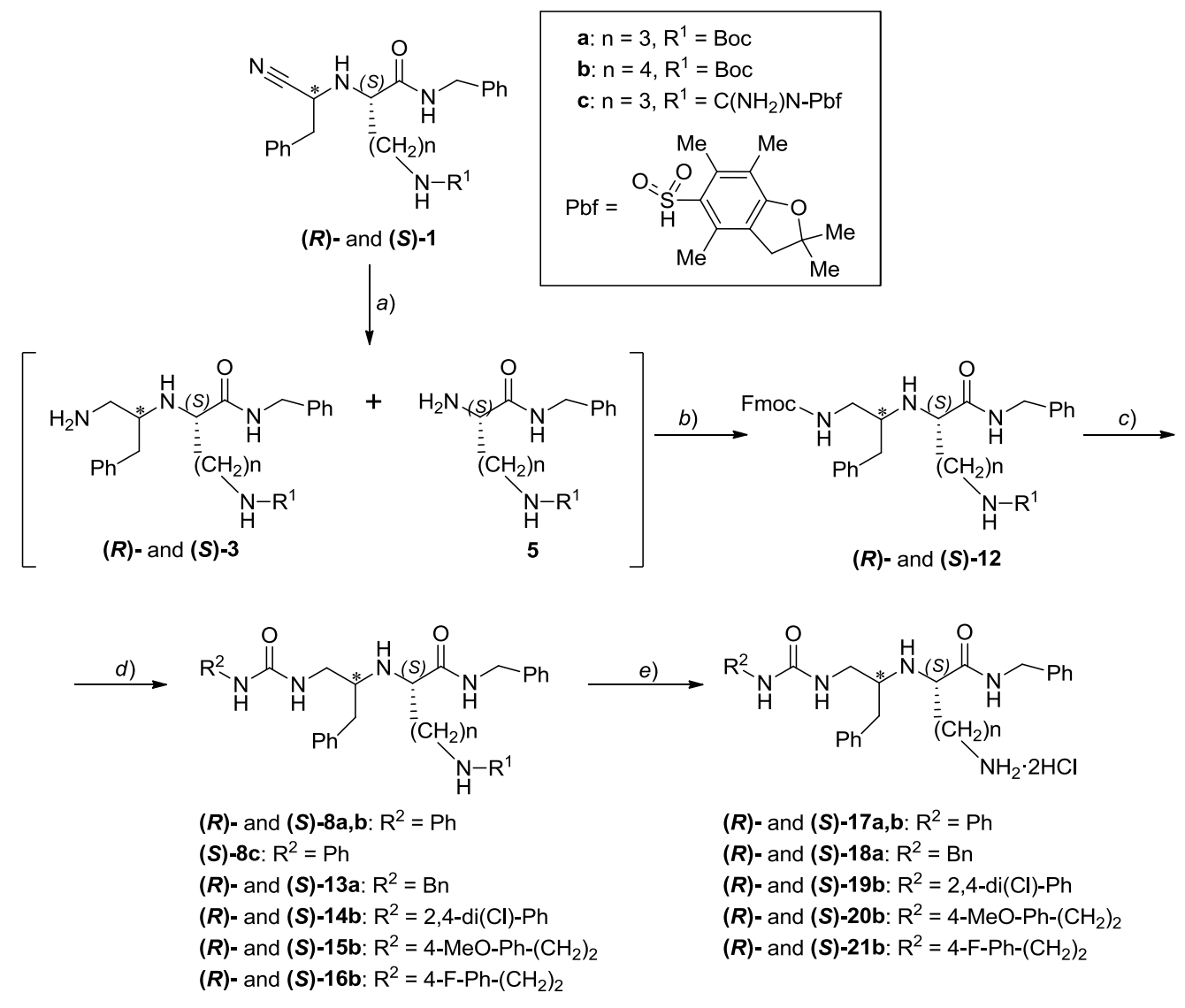

Scheme 2. Reagents and conditions: (a) $\mathrm{NH}_{2} \mathrm{NH}_{2} \cdot \mathrm{H}_{2} \mathrm{O}$, Raney $\mathrm{Ni}, \mathrm{MeOH}, 65^{\circ} \mathrm{C}$. (b) Fmoc-Cl, propylene oxide, $\mathrm{CH}_{3} \mathrm{CN}, 0^{\circ} \mathrm{C}$. (c) $\mathrm{Et}_{2} \mathrm{NH}, \mathrm{CH}_{2} \mathrm{Cl}_{2}$, rt. (d) $\mathrm{R}^{2} \mathrm{NCO}, \mathrm{CH}_{2} \mathrm{Cl}_{2}, 0^{\circ} \mathrm{C}$. (e) $3 \mathrm{M} \mathrm{HCl}$, EtOAc, rt. 
The Fmoc-removal in 12a-c, followed by reaction with different aromatic isocyanates (Scheme 2) led to the desired ureas $\mathbf{8}$ and 13-16 in significantly higher overall yields than by the direct synthesis of the urea after the cyano-reduction (compare yields of methods A and B in Table 2).

\section{Table 2}

Optimization of the synthesis of ureas 8,9 and 13-17

\begin{tabular}{|c|c|c|c|c|c|}
\hline \multirow[t]{2}{*}{ Amino nitrile } & \multicolumn{2}{|l|}{ Diamine $^{a}$} & \multicolumn{3}{|c|}{ Urea $^{b}$} \\
\hline & $\mathrm{N}^{\mathrm{o}}$ & $\%$ & $\mathrm{~N}^{\mathrm{o}}$ & $\mathrm{A}(\%)^{c}$ & $\mathrm{~B}(\%)^{d}$ \\
\hline$(\boldsymbol{R S}) \mathbf{- 1 \mathbf { a }}(1: 1)^{e}$ & $(\boldsymbol{R S}) \mathbf{- 3 \mathbf { a }}(1: 3)^{e}$ & 74 & $(\boldsymbol{R S})-\mathbf{8 a}(1: 3)^{e}$ & 36 & 52 \\
\hline$(R)-1 \mathbf{a}$ & $(\boldsymbol{R}) \mathbf{- 3 a}$ & 55 & $(R)-8 \mathbf{a}$ & - & 17 \\
\hline$(S)-1 \mathbf{a}$ & $(S)-3 \mathbf{a}$ & 92 & $(S)-8 \mathbf{a}$ & - & 67 \\
\hline$(\boldsymbol{R S})-\mathbf{1 b}(1: 1)^{e}$ & $(\boldsymbol{R S})-\mathbf{3 b}(1: 1.3)^{e}$ & 73 & $(\boldsymbol{R S}) \mathbf{- 8 b}(1: 1.3)^{e}$ & 21 & 44 \\
\hline$(R)-1 b$ & $(\boldsymbol{R})-\mathbf{3 b}$ & 60 & $(R)-8 b$ & - & 44 \\
\hline$(S)-1 \mathrm{~b}$ & $(S)-3 b$ & 87 & $(S)-8 b$ & - & 57 \\
\hline$(\boldsymbol{R S})-\mathbf{1 c}(1: 3)^{e}$ & $(S)-3 c$ & 64 & $(S)-8 \mathrm{c}$ & - & 25 \\
\hline$(R)-1 \mathbf{a}$ & $(\boldsymbol{R})-\mathbf{3 a}$ & 55 & $(R)-13 a$ & - & 25 \\
\hline$(S)-\mathbf{1 a}$ & $(S)-3 \mathbf{a}$ & 92 & $(S)-13 a$ & - & 51 \\
\hline$(R)-1 b$ & $(\boldsymbol{R})-\mathbf{3 b}$ & 60 & $(R)-14 b$ & - & 40 \\
\hline$(S)-1 b$ & $(S)-3 b$ & 87 & $(S)-14 b$ & - & 52 \\
\hline$(R)-1 b$ & $(\boldsymbol{R})-\mathbf{3 b}$ & 60 & $(R)-15 b$ & - & 42 \\
\hline$(S)-1 b$ & $(S)-3 b$ & 87 & $(S)-15 b$ & - & 61 \\
\hline$(R)-1 b$ & $(R)-3 b$ & 60 & $(R)-16 b$ & - & 40 \\
\hline$(S)-1 b$ & $(S)-\mathbf{3 b}$ & 87 & $(S)-16 b$ & - & 62 \\
\hline$(\boldsymbol{R S}) \mathbf{- 2} \mathbf{a}(1: 1)^{e}$ & $(\boldsymbol{R S})-\mathbf{4 a}(1: 1)^{e}$ & 74 & $(\boldsymbol{R S})-\mathbf{9 a}(1: 1)^{e}$ & 27 & - \\
\hline$(\boldsymbol{R S})-\mathbf{2 b}(1: 1)^{e}$ & $(\boldsymbol{R S})-\mathbf{4 b}(1: 1)^{e}$ & 74 & $(\boldsymbol{R S})-\mathbf{9 b}(1: 1)^{e}$ & 29 & - \\
\hline
\end{tabular}

${ }^{a}$ Yields determined by HPLC-MS analyses.

${ }^{b}$ Isolated yields.

${ }^{c}$ Overall yield of reduction, followed by reaction with the corresponding isocyanate (Method A).

${ }^{d}$ Overall yield of reduction, followed by sequential Fmoc-protection, isolation, Fmoc-deprotection, and reaction with the corresponding isocyanate (Method B).

${ }^{e}(R: S)$-epimer ratio.

Removal of the $N$-Boc protection of the basic side chain in the Orn and Lys derived ureas 8a,b, 9a,b and 13a,b-16b by treatment with $3 \mathrm{M} \mathrm{HCl}$ solution in EtOAc, yielded the corresponding deprotected compounds 11a,b and 17a,b-21b (Schemes 1 and 2). In view of 
the poor biological results of these deprotected ornithine and lysine derivatives (see below) and the low overall yield of the arginine-derived urea $\mathbf{8 c}$, this urea was not deprotected.

The lysine-derived Fmoc protected diamine 12b was also used for the synthesis of the PAR1 reference antagonist RWJ-58259 analogue (S)-25b (Scheme 3). This urea was synthesized by applying our recently reported procedure for the preparation of RWJ-58259 [50]. This procedure involves the in situ formation of the indazole-derived isocyanate $\mathbf{2 3}$, by reaction of the required 6-amino-indazole 22 with triphosgene in the presence of propylene oxide as $\mathrm{HCl}$ acceptor, followed by reaction with the deprotected diamines $(\boldsymbol{R})$ - and $(\boldsymbol{S})-\mathbf{3 b}$. Interestingly, as commented for the reduction of the starting amino nitriles, the application of this methodology gave considerably higher yield for $(\boldsymbol{S})$-24b $[85 \%$ overall from $(\boldsymbol{S})$-12b] than for its epimer $(\boldsymbol{R})-\mathbf{2 4 b}$ [20\% overall yield from $(\boldsymbol{R})-\mathbf{1 2 b}$ ]. Due to this low yield, this epimer was reserved for the biological evaluation and was not deprotected.

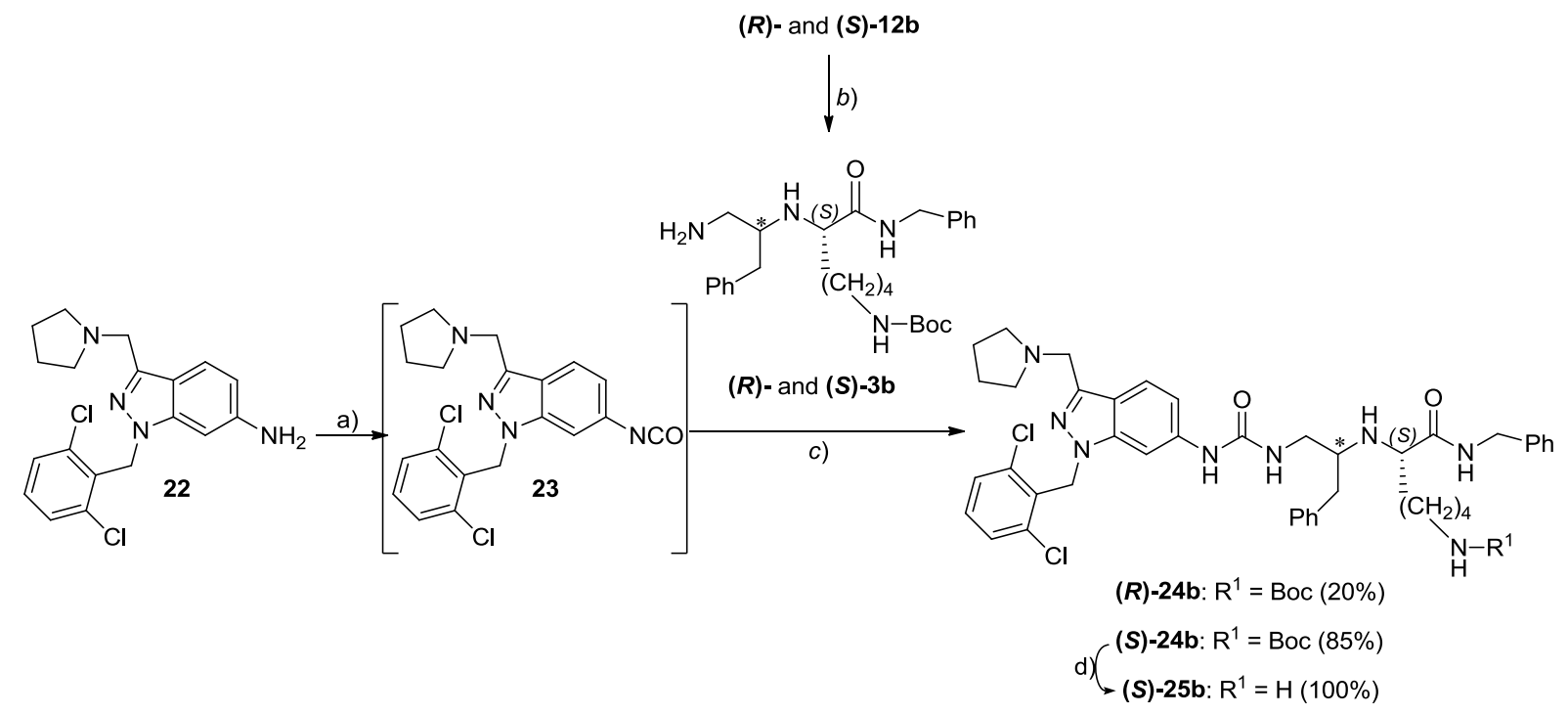

Scheme 3. Reagents and conditions: (a) $\left(\mathrm{CCl}_{3} \mathrm{O}\right)_{2} \mathrm{CO}$, propylene oxide, THF, $0{ }^{\circ} \mathrm{C}$. (b) $\mathrm{Et}_{2} \mathrm{NH}, \mathrm{CH}_{2} \mathrm{Cl}_{2}, \mathrm{rt}(c)$ THF, $0{ }^{\circ} \mathrm{C}$. (d) $3 \mathrm{M} \mathrm{HCl}$, EtOAc, rt.

The optimized method B of synthesis of urea derivatives $\mathbf{1 7 a}$ was similarly applied to the synthesis of the thiourea analogues $(\boldsymbol{R})$ - and $(\boldsymbol{S})-\mathbf{2 7} \mathbf{a}$, by replacing the phenylisocyanate by phenylisothiocyanate (Scheme 4). 


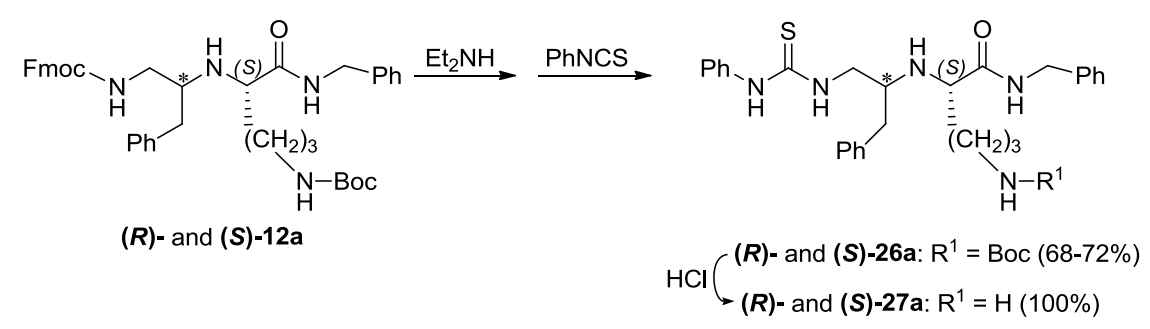

Scheme 4. Synthesis of thiourea derivatives

\subsection{Biological evaluation}

Since PAR1 is mainly expressed in platelets, to evaluate the PAR1 antagonist activity, all new compounds were screened as inhibitors of human platelet aggregation induced by a 30 $\mu \mathrm{M}$ concentration of the PAR1 agonist SFLLRN. The antagonist RWJ-58259 was used as a reference. At a $10 \mu \mathrm{M}$ concentration this antagonist inhibited $98 \%$ the platelet aggregation. All compounds were tested at an initial concentration of $0.1 \mathrm{mg} / \mathrm{mL}(\approx 150 \mu \mathrm{M})$. Unfortunately, none of the deprotected ureas or thioureas inhibited the platelet aggregation. However, as shown in Figure 2, some of the protected ureas, and particularly the Fmoc intermediates 12, showed significant inhibition. Thus, the Fmoc derivatives $(\boldsymbol{S})$-12a and $(\boldsymbol{S})$ 12c inhibited a $51 \%$ and a $36 \%$, respectively, the platelet aggregation. Among the protected ureas and thioureas, the most potent were the urea $(\boldsymbol{R})-\mathbf{8 b}$ and the thiourea $(S)-\mathbf{2 6 a}$, which showed 38 and $33 \%$ of inhibition, respectively. Although these results do not allow to establish defined structure-activity relationships, it seems that in the Fmoc derivatives $\mathbf{1 2}$ and thioureas 26a, the $(S)$-epimers showed higher inhibition \% than the respective $(R)$. However, in the case of the urea derivatives there is not a clear stereochemistry-activity relationship, as the results varied depending on the urea substitution. At this position, the phenyl group was the best (compare ureas $\mathbf{8 b}$ with $\mathbf{1 4 b - 1 6 b}$ and $\mathbf{2 4 b}$ ). Respecting the influence of the basic amino acid moiety, from the comparison of analogue $(S)$-epimers, in the Fmoc-derivatives 12, the order of activity was $\operatorname{Orn}(\mathbf{a})>\operatorname{Arg}(\mathbf{c})>\operatorname{Lys}(\mathbf{b})$, while in the urea-derivatives 8 , the order was $\operatorname{Arg}(\mathbf{c}) \geq \operatorname{Lys}(\mathbf{b})>\operatorname{Orn}(\mathbf{a})$.

In the structural comparison of the inactive indazole-derived urea $(S)-25 \mathbf{b}$ with the family of peptidomimetic urea PAR1 antagonists, to which the reference antagonist RWJ-58259 belongs [51], the main difference is the replacement of the di(F)-Phe residue of RWJ-58259 by the 1-amino-3-phenyl-propan-2-yl moiety in $(\boldsymbol{S})-\mathbf{2 5 b}$ (see Figure 1). This replacement involves the loss of the carbonyl group of the peptide bond, which could be important for the 
interaction with the PAR1 receptor, participating in H-bond formation or for the appropriate orientation of the pharmacophoric groups.

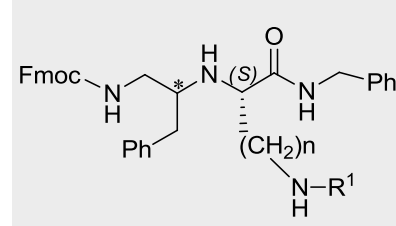

Fmoc-DERIVATIVES

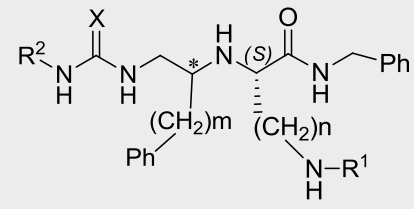

$\operatorname{UREAS}(\mathrm{X}=0)$
THIOUREAS $(\mathrm{X}=\mathrm{S})$

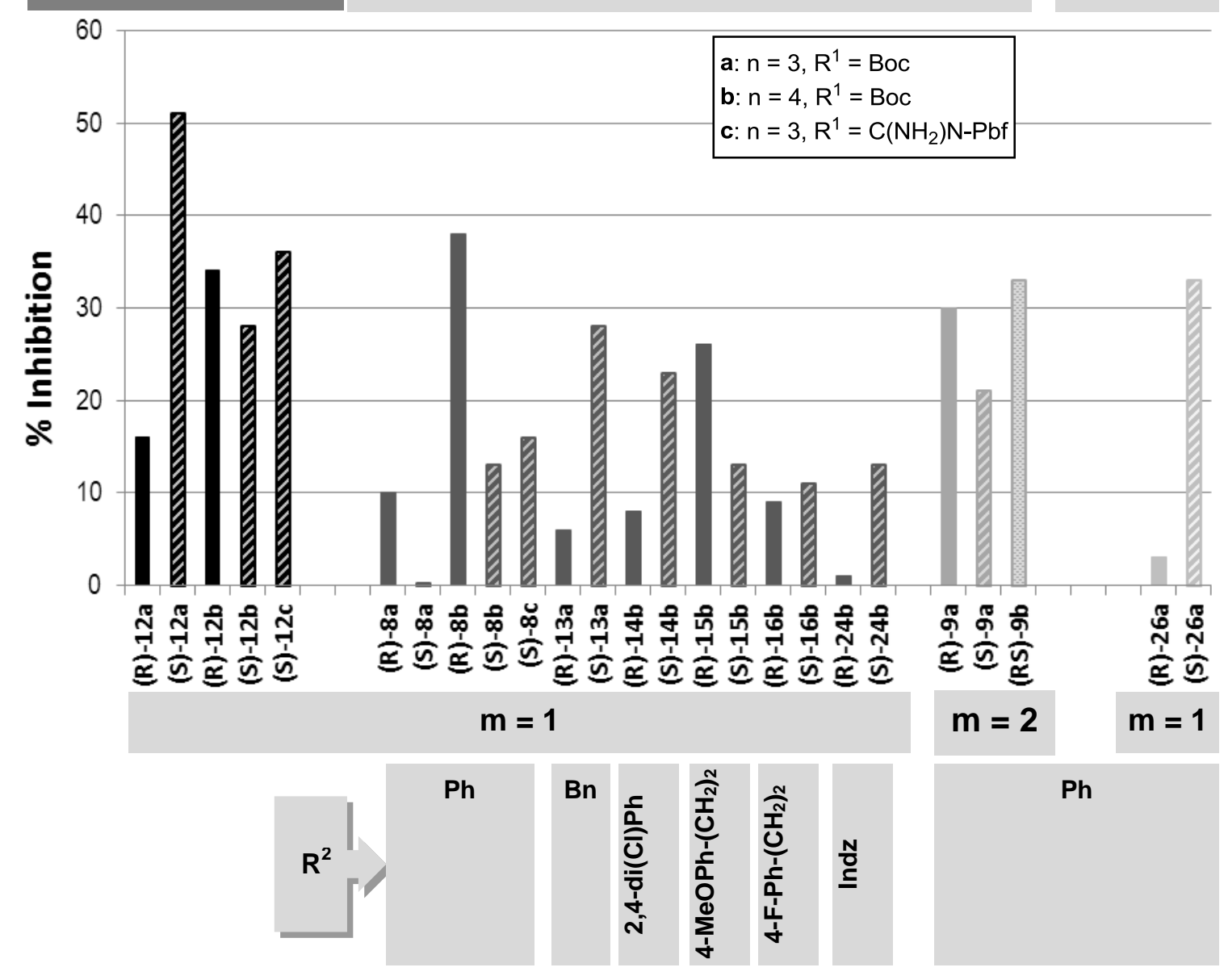

Fig. 2. Inhibition (\%) of human platelet aggregation induced by a $30 \mu \mathrm{M}$ concentration of SFLLRN

\section{Conclusion}

A series of peptide-based ureas and thioureas, including analogues of the PAR1 reference antagonist RWJ-58259, has been designed and synthesized as potential PAR1 antagonists. A DOS strategy has been applied for the synthesis of these urea derivatives, consisting of 
reduction of basic amino acid-derived amino nitriles, followed by reaction with diverse isocyanates or isothiocyanates. To evaluate the PAR1 antagonist activity, all new synthesized compounds have been screened as inhibitors of human platelet aggregation induced by the PAR1 agonist SFLLRN. Although none of the designed peptide-based ureas inhibited aggregation, some of their protected derivatives showed moderate antiaggregant activity. These results could be a good clue for the search of new potent PAR1 antagonists and to explore the thrombin/PAR1 interaction.

\section{Experimental}

\subsection{General}

All reagents were of commercial quality. Solvents were dried and purified by standard methods. Analytical TLC was performed on aluminum sheets coated with a $0.2 \mathrm{~mm}$ layer of silica gel $60 \mathrm{~F}_{254}$. Silica gel 60 (230-400 mesh) was used for flash chromatography. Analytical RP-HPLC was performed on a Sunfire $C_{18}(4.6 \times 150 \mathrm{~mm}, 3.5 \mu \mathrm{m})$ column, with a flow rate of $1 \mathrm{~mL} / \mathrm{min}$, using a tunable UV detector set at 214 and $254 \mathrm{~nm}$ and a (10-100\%, $30 \mathrm{~min}$ ) gradient of $\mathrm{CH}_{3} \mathrm{CN}$ (solvent A) and $0.05 \%$ TFA in $\mathrm{H}_{2} \mathrm{O}$ (solvent B) as mobile phase. HPLCMS was performed on a Sunfire $\mathrm{C}_{18}(4.6 \times 50 \mathrm{~mm}, 3.5 \mu \mathrm{m})$ column at $30^{\circ} \mathrm{C}$, with a flow rate of $1 \mathrm{~mL} / \mathrm{min}$. (10-100\%, $5 \mathrm{~min}$ ) Gradient of $\mathrm{CH}_{3} \mathrm{CN}$ with $0.08 \%$ of formic acid (solvent $\mathrm{A}$ ) in $0.1 \%$ of formic acid in $\mathrm{H}_{2} \mathrm{O}$ (solvent $\mathrm{B}$ ) was used as mobile phase. Electrospray in positive mode was used for ionization. Melting points were taken on a Mettler Toledo M170 apparatus and are uncorrected. Elemental analyses were obtained on a CH-O-RAOID apparatus. Optical rotations were determined in a Perkin Elmer 141 polarimeter. NMR spectra were recorded using Varian Inova 300, Varian Inova or Mercury 400, and Varian Unity 500 spectrometers. The NMR spectra assignment was based on COSY, HSQC, and HMBC spectra.

4.2. General procedure for the synthesis of basic amino acid-derived ureas. Method A. Synthesis of ureas $(\boldsymbol{R S})-\mathbf{8 a}, \mathbf{b}$ and $\mathbf{- 9 a}, \mathbf{b}$, and $(\boldsymbol{R})-$ and $(\boldsymbol{S})-\mathbf{9 a}$

Raney Ni $(30 \mathrm{mg})$ and hydrazine monohydrate $(0.186 \mathrm{~mL}, 6 \mathrm{mmol})$ were added to a solution of the corresponding amino nitrile $(\boldsymbol{R S}) \mathbf{- 1 a}, \mathbf{b}$ and $(\boldsymbol{R} \boldsymbol{S}) \mathbf{- 2 a}, \mathbf{b}(0.3 \mathrm{mmol})$ in $\mathrm{MeOH}$ $(20 \mathrm{~mL})$ and the resulting reaction mixture was refluxed for $5 \mathrm{~min}$. Then, the mixture was filtered through celite, this was washed with $\mathrm{MeOH}(3 \times 75 \mathrm{~mL})$, and the filtrates were evaporated to dryness. The residue was dissolved in dry $\mathrm{CH}_{2} \mathrm{Cl}_{2}(10 \mathrm{~mL})$ and the solution was 
cooled at $0^{\circ} \mathrm{C}$. The corresponding isocyanate $(0.3 \mathrm{mmol})$ was added to the solution that was stirred at this temperature for $2 \mathrm{~h}$. Then, the solvent was evaporated and the residue was dissolved in EtOAc $(50 \mathrm{~mL})$. This solution was successively washed with $\mathrm{H}_{2} \mathrm{O}(20 \mathrm{~mL})$ and brine $(20 \mathrm{~mL})$, dried over $\mathrm{Na}_{2} \mathrm{SO}_{4}$, and evaporated to dryness. The residue was purified by flash chromatography, using 20-100\% EtOAc gradient in hexane as eluant to afford the ureas $(\boldsymbol{R S})-\mathbf{8 a}, \mathbf{b}$ and $(\boldsymbol{R S})-\mathbf{9 a}, \mathbf{b}$. Only both epimers of the ornithine-derived ureas $(\boldsymbol{R})-\mathbf{9 a}$ and $(\boldsymbol{S})-\mathbf{9 a}$ could be separated in this purification. The two epimers of $(\boldsymbol{R S})-\mathbf{8 a}, \mathbf{b}$ were obtained as optical pure compounds from each epimer of the starting amino nitrile $(\boldsymbol{R})-\mathbf{1 a}, \mathbf{b}$ and $(\boldsymbol{S})-\mathbf{1 a}, \mathbf{b}$, by applying method B described below. The characterization data of $(\boldsymbol{R})-\mathbf{8 a}, \mathbf{b}$ and $(\boldsymbol{S})-\mathbf{8 a}, \mathbf{b}$ are also described below.

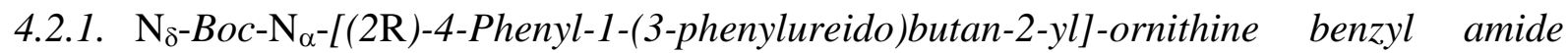
$[(\boldsymbol{R})-9 \mathbf{a}]$

Foam $(23 \mathrm{mg}, 13 \%)$; HPLC $t_{\mathrm{R}} 18.54 ;[\alpha]_{\mathrm{D}}{ }^{20}+7.5(c 0.9, \mathrm{MeOH}) ;{ }^{1} \mathrm{H}$ NMR $(400 \mathrm{MHz}$, $\left.\mathrm{CDCl}_{3}\right) \delta 1.40(\mathrm{~s}, 9 \mathrm{H}, \mathrm{Boc}), 1.29-1.81$ [m, 6H, 3-H (Bu), $\gamma$ - and $\left.\beta-\mathrm{H}(\mathrm{Orn})\right], 2.49-2.78$ [m, 3H, 2-H and 4-H (Bu)], 2.95-3.43 [m, 4H, 1-H (Bu), $\alpha-$ and $\delta-\mathrm{H}(\mathrm{Orn})], 4.32\left[\mathrm{~m}, 2 \mathrm{H}, \mathrm{CH}_{2}(\mathrm{Bn})\right]$, 4.92 (bs, 1H, NH-Boc), 5.86, and 7.67 (2 bs, 2H, HNCONH), 6.95-7.56 (m, 16 H, Ph and NH$\mathrm{Bn}) ;{ }^{13} \mathrm{C} \mathrm{NMR}\left(100 \mathrm{MHz}, \mathrm{CDCl}_{3}\right) \delta 28.6\left(\mathrm{CH}_{3}, \mathrm{Boc}\right.$ and $\left.\mathrm{CH}_{2}, \mathrm{C}_{\gamma}\right), 31.6\left(\mathrm{CH}_{2}, \mathrm{C}_{\beta}\right), 32.3\left(\mathrm{CH}_{2}\right.$, $\left.\mathrm{C}_{4}\right), 35.7\left(\mathrm{CH}_{2}, \mathrm{C}_{3}\right), 39.5\left(\mathrm{CH}_{2}, \mathrm{C}_{\delta}\right), 43.1\left(\mathrm{CH}_{2}, \mathrm{C}_{1}\right), 43.5\left[\mathrm{CH}_{2},(\mathrm{Bn})\right], 57.1\left(\mathrm{CH}, \mathrm{C}_{2}\right), 58.3$ $\left(\mathrm{CH}, \mathrm{C}_{\alpha}\right), 79.8(\mathrm{C}, \mathrm{Boc}), 119.8-129.2(15 \mathrm{CH}, \mathrm{Ph}), 138.5,139.6$ and $141.9(3 \mathrm{C}, \mathrm{Ph}), 156.2$ (HNCONH), $157.1(\mathrm{CO}, \mathrm{Boc}), 175.1(\mathrm{CONH})$; ES-MS $\mathrm{m} / z[\mathrm{M}+1]^{+}$calcd. for $\mathrm{C}_{34} \mathrm{H}_{45} \mathrm{~N}_{5} \mathrm{O}_{4}$, 688.35; found 688.17 (100\%); Anal. calcd. for $\mathrm{C}_{34} \mathrm{H}_{45} \mathrm{~N}_{5} \mathrm{O}_{4}$ : C, 69.48; H, 7.72; N, 11.92 . Found: C, 69.67; H, 7.86; N, 11.78.

4.2.2. $\mathrm{N}_{\delta}$-Boc- $\mathrm{N}_{\alpha}-[(2 \mathrm{~S})-4-P h e n y l-1-(3-p h e n y l u r e i d o) b u t a n-2-y l]$-ornithine benzyl amide $[(S)-9 a]$

Foam $(25 \mathrm{mg}, 14 \%)$; HPLC $t_{\mathrm{R}} 18.89 ;[\alpha]_{\mathrm{D}}{ }^{20}+5\left(c\right.$ 1, MeOH); ${ }^{1} \mathrm{H}$ NMR (400 MHz, $\left.\mathrm{CDCl}_{3}\right) \delta 1.42$ (s, 9H, Boc), 1.46-1.80 [m, 6H, 3-H (Bu), $\gamma-$ and $\left.\beta-\mathrm{H}(\mathrm{Orn})\right], 2.51-2.63$ [m, $3 \mathrm{H}, 2-\mathrm{H}$ and 4-H (Bu)], 2.92-3.31 [m, 4H, 1-H (Bu), $\alpha-$ and $\delta-\mathrm{H}(\mathrm{Orn})], 4.26[\mathrm{dd}, 1 \mathrm{H}, \mathrm{J}=6$ and $15 \mathrm{~Hz}, \mathrm{CH}_{2}(\mathrm{Bn})$ ], 4. 31 [dd, $1 \mathrm{H}, \mathrm{J}=6$ and $15 \mathrm{~Hz}, \mathrm{CH}_{2}(\mathrm{Bn})$ ], 4.73 (bs, 1H, NH-Boc), 5.84, and 7.70 (2 bs, $2 \mathrm{H}, \mathrm{HNCONH}), 6.92-7.52$ (m, $16 \mathrm{H}, \mathrm{Ph}$ and $N H-\mathrm{Bn}) ;{ }^{13} \mathrm{C} \mathrm{NMR}(100$ $\left.\mathrm{MHz}, \mathrm{CDCl}_{3}\right) \delta 28.6\left(\mathrm{CH}_{2}, \mathrm{C}_{\gamma}\right), 28.7\left(\mathrm{CH}_{3}, \mathrm{Boc}\right), 32.2\left(\mathrm{CH}_{2}, \mathrm{C}_{\beta}\right), 32.4\left(\mathrm{CH}_{2}, \mathrm{C}_{4}\right), 35.6\left(\mathrm{CH}_{2}\right.$, 
$\left.\mathrm{C}_{3}\right), 40.3\left(\mathrm{CH}_{2}, \mathrm{C}_{\delta}\right), 42.5\left(\mathrm{CH}_{2}, \mathrm{C}_{1}\right), 43.4\left[\mathrm{CH}_{2},(\mathrm{Bn})\right], 57.4\left(\mathrm{CH}, \mathrm{C}_{2}\right), 59.9\left(\mathrm{CH}, \mathrm{C}_{\alpha}\right), 80.1(\mathrm{C}$, Boc), 119.6-129.2 (15CH, Ph), 138.6, 139.6 and 141.9 (3C, Ph), 156.7 (HNCONH), 157.2 (CO, Boc), $174.9(\mathrm{CONH})$; ES-MS $m / z[\mathrm{M}+1]^{+}$calcd. for $\mathrm{C}_{34} \mathrm{H}_{45} \mathrm{~N}_{5} \mathrm{O}_{4}, 688.35$; found 688.27 (100\%); Anal. calcd. for $\mathrm{C}_{34} \mathrm{H}_{45} \mathrm{~N}_{5} \mathrm{O}_{4}$ : C, 69.48; H, 7.72; N, 11.92. Found: C, 69.59; H, 7.82; N, 11.85 .

\subsection{3. $\mathrm{N}_{\varepsilon}$-Boc- $\mathrm{N}_{\alpha}$-[4-Phenyl-1-(3-phenylureido)butan-2-yl]-lysine benzyl amide [(RS)-9b]}

Foam (52 mg, $29 \%)$; HPLC $t_{\mathrm{R}} 18.70[(\boldsymbol{R})-9 \mathbf{b}]$ and $19.06 \mathrm{~min}[(\boldsymbol{S})-\mathbf{9 b}] ;{ }^{1} \mathrm{H}$ NMR (400 $\left.\mathrm{MHz}, \mathrm{CDCl}_{3}\right) \delta 1.41$ and 1.43 (2s, 9H, Boc), 0.94-1.33 [m, 2H, $\delta-\mathrm{H}$ (Lys)], 1.50-1.85 [m, 6H, $\gamma$ - and $\beta-\mathrm{H}$ (Lys), 3-H (Bu)], 2.45-2.74 [m, 3H, 2-H and 4-H (Bu)], 2.90-3.17 [m, 2H, $\varepsilon-\mathrm{H}$ (Lys)], 3.17-3.27 [m, 1.5H, 1-H (Bu) and $\alpha-\mathrm{H}(\mathrm{Lys})], 3.38$ [m, 0.5H, 1-H (Bu)], 4.36 [m, 2H, $\mathrm{CH}_{2}(\mathrm{Bn})$ ], 4.66 and 4.84 (2bs, 1H, NH-Boc), 5.80, 7.84 and 8.10 (3 bs, 2H, HNCONH), 6.86$7.54(\mathrm{~m}, 16 \mathrm{H}, \mathrm{Ph}$ and $N H-\mathrm{Bn}) ;{ }^{13} \mathrm{C}$ NMR $\left(100 \mathrm{MHz}, \mathrm{CDCl}_{3}\right) \delta 22.9\left(\mathrm{CH}_{2}, \mathrm{C}_{\gamma}\right), 26.4$ and 28.5 $\left(\mathrm{CH}_{3}, \mathrm{Boc}\right), 29.8\left(\mathrm{CH}_{2}, \mathrm{C}_{\delta}\right), 32.1\left(\mathrm{CH}_{2}, \mathrm{C}_{4}\right), 33.0\left(\mathrm{CH}_{2}, \mathrm{C}_{\beta}\right), 34.8\left(\mathrm{CH}_{2}, \mathrm{C}_{3}\right), 40.0\left(\mathrm{CH}_{2}, \mathrm{C}_{\varepsilon}\right)$, 42.0 and $43.0\left(\mathrm{CH}_{2}, \mathrm{C}_{1}\right), 43.3\left[\mathrm{CH}_{2},(\mathrm{Bn})\right], 56.9$ and $58.8\left(\mathrm{CH}, \mathrm{C}_{2}\right), 60.1\left(\mathrm{CH}, \mathrm{C}_{\alpha}\right), 80.1(\mathrm{C}$, Boc), 119.1-128.9 (15CH, Ph), 139.1 and 141.3 (3C, Ph), 156.2 and 156.7 (2CO, Boc and HNCONH), 174.9 (CONH); ES-MS m/z $[\mathrm{M}+1]^{+}$calcd. for $\mathrm{C}_{35} \mathrm{H}_{47} \mathrm{~N}_{5} \mathrm{O}_{4}, 602.36$; found 602.27 (100\%); Anal. calcd. for $\mathrm{C}_{35} \mathrm{H}_{47} \mathrm{~N}_{5} \mathrm{O}_{4}$ : C, 69.86; H, 7.87; N, 11.64. Found: C, 69.97; H, $7.84 ; \mathrm{N}, 11.45$.

\subsection{General procedure for the synthesis of the N-Fmoc-protected amines $(\boldsymbol{R})-\mathbf{1 2 a}, \mathbf{b}$ and $(\boldsymbol{S})-$ 12a-c}

Raney Ni (30 mg) and hydrazine monohydrate $(0.186 \mathrm{~mL}, 6 \mathrm{mmol})$ were added to a solution of the corresponding amino nitrile $(\boldsymbol{R})$ - and $(\boldsymbol{S})-\mathbf{1 a - c}(0.3 \mathrm{mmol})$ in $\mathrm{MeOH}(20 \mathrm{~mL})$ and the resulting reaction mixture was refluxed for $5 \mathrm{~min}$. Then, the mixture was filtered through celite, this was washed with $\mathrm{MeOH}(3 \times 75 \mathrm{~mL})$, and the filtrates were evaporated to dryness. The respective crude reaction mixture of amines $(\boldsymbol{R})-\mathbf{3 a}, \mathbf{b}$ and $(\boldsymbol{S})-\mathbf{3 a} \mathbf{\mathbf { a }}$-c impurified with 5a-c was dissolved in $\mathrm{CH}_{3} \mathrm{CN}(10 \mathrm{~mL})$ and cooled at $0^{\circ} \mathrm{C}$. Propylene oxide $(56 \mu \mathrm{L}, 8$ mmol) and Fmoc-Cl (78 mg, $0.3 \mathrm{mmol})$ were added. The solution was stirred for $2 \mathrm{~h}$. Then, the solvent was evaporated and the residue was dissolved in EtOAc $(50 \mathrm{~mL})$. This solution was successively washed with $\mathrm{H}_{2} \mathrm{O}(20 \mathrm{~mL})$ and brine $(20 \mathrm{~mL})$, dried over $\mathrm{Na}_{2} \mathrm{SO}_{4}$, and evaporated to dryness. The residue was purified by flash chromatography, using 10-40\% 
EtOAc gradient in hexane as eluant to give the corresponding Fmoc-protected amine $(\boldsymbol{R})$ 12a,b and $(S)-12 a-c$.

4.3.1. $\mathrm{N}_{\delta}$-Boc- $\mathrm{N}_{\alpha}-[(2 \mathrm{R})-3-P h e n y l-1-($ Fmoc $)$ amino-propan-2-yl)-ornithine benzyl amide $[(\boldsymbol{R})$ 12a]

Foam (112 mg, 55\%); HPLC $t_{\mathrm{R}} 20.56 \mathrm{~min} ;[\alpha]_{\mathrm{D}}{ }^{20}+7.1(c 1, \mathrm{MeOH}) ;{ }^{1} \mathrm{H}$ NMR $(400 \mathrm{MHz}$, $\left.\mathrm{CDCl}_{3}\right) \delta$ 1.10-1.98 [m, 4H, $\beta-\mathrm{H}$ and $\left.\gamma-\mathrm{H}(\mathrm{Orn})\right], 1.36$ (s, 9H, Boc), 2.76-3.14 [m, 5H, 2-H, 3$\mathrm{H}(\mathrm{Pr})$ and $\delta-\mathrm{H}(\mathrm{Orn})], 3.24[\mathrm{~m}, 1 \mathrm{H}, \alpha-\mathrm{H}(\mathrm{Orn})], 3.26-3.55[\mathrm{~m}, 1 \mathrm{H}, 1-\mathrm{H}(\mathrm{Pr})], 4.19[\mathrm{t}, 1 \mathrm{H}, J=7$ $\mathrm{Hz}, \mathrm{CH}$ (Fmoc)], 4.37 [d, 2H, J=7 Hz, $\mathrm{CH}_{2}$ (Fmoc)], 4.42 [dd, 2H, J=5 and 15 Hz, $\mathrm{CH}_{2}$ (Bn)], 4.71 (bs, $1 \mathrm{H}, N H$-Boc), 5.75 (1bs, 1H, NH-Fmoc), 7.14-7.76 (m, 19H, aromatics and NH-Bn); ${ }^{13} \mathrm{C}$ NMR $\left(100 \mathrm{MHz}, \mathrm{CDCl}_{3}\right) \delta 26.0\left(\mathrm{CH}_{2}, \mathrm{C}_{\gamma}\right), 28.3\left(3 \mathrm{CH}_{3}, \mathrm{Boc}\right), 30.7\left(\mathrm{CH}_{2}, \mathrm{C}_{\beta}\right), 39.1\left(\mathrm{CH}_{2}\right.$, $\left.\mathrm{C}_{3}\right), 39.5\left(\mathrm{CH}_{2}, \mathrm{C}_{\delta}\right), 43.1\left(\mathrm{CH}_{2}, \mathrm{Bn}\right), 44.6\left(\mathrm{CH}_{2}, \mathrm{C}_{1}\right), 47.1(\mathrm{CH}, \mathrm{Fmoc}), 59.6\left(\mathrm{CH}, \mathrm{C}_{2}\right), 60.2$ $\left(\mathrm{CH}, \mathrm{C}_{\alpha}\right), 66.5\left(\mathrm{CH}_{2}, \mathrm{Fmoc}\right), 79.2(\mathrm{C}, \mathrm{Boc}), 119.9-143.9$ (18CH and 6C, aromatics), 156.2 (CO, Boc), 156.8 (CO, Fmoc), $174.2(\mathrm{CONH})$; ES-MS m/z [M+1] $]^{+}$calcd for $\mathrm{C}_{41} \mathrm{H}_{48} \mathrm{~N}_{4} \mathrm{O}_{5}$, 677.36; found 677.59 (100\%); Anal. calcd. for $\mathrm{C}_{41} \mathrm{H}_{48} \mathrm{~N}_{4} \mathrm{O}_{5}$ : C, 72.76; H, 7.15; N, 8.28. Found: C, 72.93; H, 7.34; N, 8.05.

4.3.2. $\mathrm{N}_{\delta}-$ Boc- $\mathrm{N}_{\alpha}-[(2 \mathrm{~S})-3-P h e n y l-1-($ Fmoc)amino-propan-2-yl)-ornithine benzyl amide $[(\boldsymbol{S})$ 12a]

Foam (162 mg, 80\%); HPLC $t_{\mathrm{R}} 21.26 \mathrm{~min} ;[\alpha]_{\mathrm{D}}{ }^{20}+15.4(c 1, \mathrm{MeOH}) ;{ }^{1} \mathrm{H}$ NMR $(400 \mathrm{MHz}$, $\left.\mathrm{CDCl}_{3}\right) \delta 1.41$ (s, 9H, Boc), 1.46-1.59 [m, 4H, $\beta-\mathrm{H}$ and $\left.\gamma-\mathrm{H}(\mathrm{Orn})\right], 2.41-2.71[(\mathrm{~m}, 2 \mathrm{H}, 3-\mathrm{H}$ (Pr)], 2.77 [m, 1H, 2-H (Pr)] , 2.97-3.16 [m, 2H, $\delta-\mathrm{H}(\mathrm{Orn})], 3.16-3.43$ [m, 3H, $\alpha-\mathrm{H}$ and 1-H (Orn)], 3.83 ]dd, 2H, J=7, 15 Hz, $\mathrm{CH}_{2}$ (Bn)], 4.20 [t, 1H, J=6.5 Hz, CH (Fmoc)], 4.35 [dd, 2H, $\left.J=5,15 \mathrm{~Hz}, \mathrm{CH}_{2}(\mathrm{Bn})\right], 4.42$ [d, 2H, J=6.5 Hz, $\mathrm{CH}_{2}$ (Fmoc)], 4.75 (bs, 1H, NH-Boc), 5.32 (1bs, 1H, NH-Fmoc), 6.91 (1bs, 1H, NH-Bn), 7.04-7.77 (m, 19H, aromatics); ${ }^{13} \mathrm{C}$ NMR (100 $\left.\mathrm{MHz}, \mathrm{CDCl}_{3}\right) \delta 26.3\left(\mathrm{CH}_{2}, \mathrm{C}_{\gamma}\right), 28.4\left(3 \mathrm{CH}_{3}, \mathrm{Boc}\right), 30.9\left(\mathrm{CH}_{2}, \mathrm{C}_{\beta}\right), 39.6\left(\mathrm{CH}_{2}, \mathrm{C}_{3}\right), 40.1\left(\mathrm{CH}_{2}\right.$, $\left.\mathrm{C}_{\delta}\right), 42.7\left(\mathrm{CH}_{2}, \mathrm{Bn}\right), 43.1\left(\mathrm{CH}_{2}, \mathrm{C}_{1}\right), 47.2(\mathrm{CH}, \mathrm{Fmoc}), 58.7\left(\mathrm{CH}, \mathrm{C}_{2}\right), 59.1\left(\mathrm{CH}, \mathrm{C}_{\alpha}\right), 66.7$ ( $\mathrm{CH}_{2}$, Fmoc), 79.3 (C, Boc), 119.9-143.8 (18CH and 6C, aromatics), 156.3 (CO, Boc), 157.1 (CO, Fmoc), $174.2(\mathrm{CONH})$; ES-MS $m / z[\mathrm{M}+1]^{+}$calcd for $\mathrm{C}_{41} \mathrm{H}_{48} \mathrm{~N}_{4} \mathrm{O}_{5}, 677.36$; found 677.59 (100\%); Anal. calcd. for $\mathrm{C}_{41} \mathrm{H}_{48} \mathrm{~N}_{4} \mathrm{O}_{5}$ : C, 72.76; H, 7.15; N, 8.28. Found: C, 72.89; H, 7.32; $\mathrm{N}, 8.12$. 
4.3.3. $\mathrm{N}_{\varepsilon}-$ Boc- $\mathrm{N}_{\alpha}-[(2 \mathrm{R})-3-P h e n y l-1-($ Fmoc)amino-propan-2-yl)-lysine benzyl amide $[(\boldsymbol{R})$ 12b]

Foam (132 mg, 64\%); HPLC $t_{\mathrm{R}} 20.68 \mathrm{~min} ;[\alpha]_{\mathrm{D}}{ }^{20}+8.2(c 0.8, \mathrm{MeOH}) ;{ }^{1} \mathrm{H}$ NMR (400 $\left.\mathrm{MHz}, \mathrm{CDCl}_{3}\right) \delta$ 0.91-164 [m, 6H, $\beta-\mathrm{H}, \gamma-\mathrm{H}$ and $\delta$-H (Lys)], 1.44 (s, 9H, Boc), 2.48-2.83 [m, $3 \mathrm{H}, 2-\mathrm{H}(\mathrm{Pr})$ and $\varepsilon-\mathrm{H}(\mathrm{Lys})], 2.88-3.02$ [m, 2H, 3-H (Pr)], 3.06-3.19 [m, 3H, 1-H (Pr) and $\alpha-\mathrm{H}$ (Lys)], 4.17 [t, 1H, J=7.5 Hz, CH (Fmoc)], 4.28-4.47 [m, 4H, $\mathrm{CH}_{2}$ (Fmoc) and $\mathrm{CH}_{2}$ (Bn)], 4.60 (bs, 1H, NH-Boc), 5.18 (1bs, 1H, NH-Fmoc), 7.09 (1bs, 1H, NH-Bn), 7.11-7.75 (m, 19H, aromatics); ${ }^{13} \mathrm{C} \mathrm{NMR}\left(100 \mathrm{MHz}, \mathrm{CDCl}_{3}\right) \delta 22.4\left(\mathrm{CH}_{2}, \mathrm{C}_{\gamma}\right), 28.3\left(3 \mathrm{CH}_{3}, \mathrm{Boc}\right), 29.6$ $\left(\mathrm{CH}_{2}, \mathrm{C}_{\delta}\right), 33.1\left(\mathrm{CH}_{2}, \mathrm{C}_{\beta}\right), 38.9\left(\mathrm{CH}_{2}, \mathrm{C}_{3}\right), 39.9\left(\mathrm{CH}_{2}, \mathrm{C}_{\varepsilon}\right), 42.9\left(\mathrm{CH}_{2}, \mathrm{Bn}\right), 44.6\left(\mathrm{CH}_{2}, \mathrm{C}_{1}\right)$, 47.1 (CH, Fmoc), $59.2\left(\mathrm{CH}, \mathrm{C}_{2}\right), 61.1\left(\mathrm{CH}, \mathrm{C}_{\alpha}\right), 66.4\left(\mathrm{CH}_{2}, \mathrm{Fmoc}\right), 78.9(\mathrm{C}, \mathrm{Boc}), 119.8-$ 143.8 (18CH and 6C, aromatics), 155.9 (CO, Boc), 156.7 (NHCOO, Fmoc), 174.4 (CONH); ES-MS $m / z,[\mathrm{M}+1]^{+}$calcd for $\mathrm{C}_{42} \mathrm{H}_{50} \mathrm{~N}_{4} \mathrm{O}_{5}, 691.38$; found 691.59 (100\%); Anal. calcd. for $\mathrm{C}_{42} \mathrm{H}_{50} \mathrm{~N}_{4} \mathrm{O}_{5}$ : C, 73.02; H, 7.29; N, 8.11. Found: C, 72.89; H, 7.32; N, 8.02.

4.3.4. $\mathrm{N}_{\varepsilon}-$ Boc- $\mathrm{N}_{\alpha}-[(2 \mathrm{~S})-3-P h e n y l-1-(F m o c)$ amino-propan-2-yl)-lysine benzyl amide $[(\mathrm{S})$ 12b]

Foam (145 mg, 70\%); HPLC $t_{\mathrm{R}} 21.21 \mathrm{~min} ;[\alpha]_{\mathrm{D}}{ }^{20}+12.3(c 0.9, \mathrm{MeOH}) ;{ }^{1} \mathrm{H}$ NMR (400 $\left.\mathrm{MHz}, \mathrm{CDCl}_{3}\right) \delta 1.16-1.86[\mathrm{~m}, 6 \mathrm{H}, \beta-\mathrm{H}, \gamma-\mathrm{H}$ and $\delta-\mathrm{H}$ (Lys)], 1.42 (s, 9H, Boc), 2.42-2.68 [m, 2H, 3-H (Pr)], 2.72 [m, 1H, 2-H (Pr)], 2.88-3.44 [m, 4H, 1-H (Pr) and $\varepsilon-\mathrm{H}(\mathrm{Lys})], 3.22$ [m, 1H, $\alpha-\mathrm{H}$ (Lys)], 3.83 [dd, 1H, J= 7 and $15 \mathrm{~Hz}, \mathrm{CH}_{2}(\mathrm{Bn})$ ], 4.19 [t, 1H, J=7.5 Hz, CH (Fmoc)], 4.35 [dd, 1H, J= 7, $15 \mathrm{~Hz}, \mathrm{CH}_{2}$ (Bn)], 4.41 [d, 2H, J=7.5 Hz, $\mathrm{CH}_{2}$ (Fmoc)], 4.57 (bs, 1H, NHBoc), 5.19 (1bs, 1H, NH-Fmoc), 7.04 (1bs, 1H, NH-Bn), 7.07-7.77 (m, 19H, aromatics); ${ }^{13} \mathrm{C}$ NMR (100 MHz, $\left.\mathrm{CDCl}_{3}\right) \delta 22.5\left(\mathrm{CH}_{2}, \mathrm{C}_{\gamma}\right), 28.4\left(3 \mathrm{CH}_{3}, \mathrm{Boc}\right), 29.8\left(\mathrm{CH}_{2}, \mathrm{C}_{\delta}\right), 33.5\left(\mathrm{CH}_{2}, \mathrm{C}_{\beta}\right)$, $39.8\left(\mathrm{CH}_{2}, \mathrm{C}_{3}\right), 41.5\left(\mathrm{CH}_{2}, \mathrm{C}_{\varepsilon}\right), 42.7\left(\mathrm{CH}_{2}, \mathrm{Bn}\right), 43.5\left(\mathrm{CH}_{2}, \mathrm{C}_{1}\right), 47.2(\mathrm{CH}, \mathrm{Fmoc}), 59.3(\mathrm{CH}$, $\left.\mathrm{C}_{2}\right), 60.2\left(\mathrm{CH}, \mathrm{C}_{\alpha}\right), 66.7$ ( $\left.\mathrm{CH}_{2}, \mathrm{Fmoc}\right), 79.1$ (C, Boc), 119.9-143.7 (18CH and 6C, aromatics), 156.2 (CO, Boc), 157.0 (CO, Fmoc), $174.4(\mathrm{CONH})$; ES-MS $\mathrm{m} / z[\mathrm{M}+1]^{+}$calcd for $\mathrm{C}_{42} \mathrm{H}_{50} \mathrm{~N}_{4} \mathrm{O}_{5}, 691.38$; found $691.59(100 \%)[\mathrm{M}+1]^{+}$; Anal. calcd. for $\mathrm{C}_{42} \mathrm{H}_{50} \mathrm{~N}_{4} \mathrm{O}_{5}: \mathrm{C}, 73.02$; H, 7.29; N, 8.11. Found: C, 73.14; H, 7.38; N, 7.96.

4.3.5. $\mathrm{N}_{\delta}-P b f-\mathrm{N}_{\alpha}-[(2 \mathrm{~S})-3-P h e n y l-1-(F m o c)$ amino-propan-2-yl)-arginine benzyl amide $[(\boldsymbol{S})$ 12c] 
Foam $(222 \mathrm{mg}, 85 \%)$; HPLC [Sunfire $\mathrm{C}_{18}, 3.9 \times 50 \mathrm{~mm}, 3.5 \mu \mathrm{m},(10-100 \%, 5 \mathrm{~min})$ gradient of solvent $\mathrm{A}$ in solvent $\mathrm{B}] t_{\mathrm{R}} 4.65 \mathrm{~min} ;[\alpha]_{\mathrm{D}}{ }^{20}+6.4(c 1, \mathrm{MeOH}) ;{ }^{1} \mathrm{H}-\mathrm{RMN}(500$

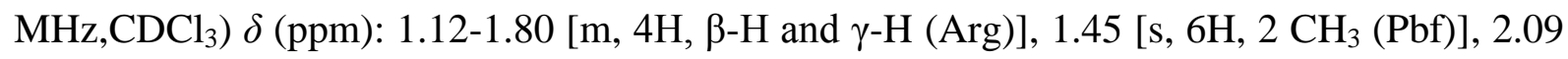
[s, 3H, $\mathrm{CH}_{3}$ (Pbf)], 2.51 [s, 3H, $\mathrm{CH}_{3}$ (Pbf)], 2.59 [s, 3H, $\left.\mathrm{CH}_{3}(\mathrm{Pbf})\right], 2.66-2.84$ [m, 2H, 1-H (Pr)]), $2.73[\mathrm{~m}, 1 \mathrm{H}, 2-\mathrm{H}(\mathrm{Pr})], 2.92\left[\mathrm{~s}, 2 \mathrm{H}, \mathrm{CH}_{2}(\mathrm{Pbf})\right], 3.02-3.55[\mathrm{~m}, 4 \mathrm{H}, 1-\mathrm{H}(\mathrm{Pr})$ and $\delta-$ $\mathrm{H}(\mathrm{Arg})], 3.38$ [m, 1H, $\alpha-\mathrm{H}(\mathrm{Arg})], 3.85$ [dd, 1H, J = 7.5 and $\left.15 \mathrm{~Hz}, \mathrm{CH}_{2}(\mathrm{Bn})\right], 4.17$ [t, 1H, J = $7 \mathrm{~Hz}, \mathrm{CH}_{2}$ (Fmoc)], 4.29 [dd, $1 \mathrm{H}, \mathrm{J}=7.5$ and $\left.15 \mathrm{~Hz}, \mathrm{CH}_{2}(\mathrm{Bn})\right], 4.25-4.53\left[\mathrm{~m}, 2 \mathrm{H}, \mathrm{CH}_{2}\right.$ (Fmoc)], 5.60 (bs, 1H, NH-Fmoc), 6.28 (bs, 3H, guanidine $\mathrm{NH}), 6.97-7.78$ (m, 15H, aromatics); ${ }^{13} \mathrm{C}-\mathrm{RMN}\left(125 \mathrm{MHz}, \mathrm{CDCl}_{3}\right) \delta$ (ppm): 12.9, 18.3, 19.7, $28.9\left(5 \mathrm{CH}_{3}, \mathrm{Pbf}\right), 25.7$ $\left(\mathrm{CH}_{2}, \mathrm{C}_{\gamma}\right), 30.1\left(\mathrm{CH}_{2}, \mathrm{C}_{\beta}\right), 31.3\left(\mathrm{CH}_{2}, \mathrm{C}_{3}\right), 40.3\left(\mathrm{CH}_{2}, \mathrm{C}_{\delta}\right), 43.1\left(\mathrm{CH}_{2}, \mathrm{C}_{1}\right), 43.6\left(\mathrm{CH}_{2}, \mathrm{Pbf}\right)$, $47.6(\mathrm{CH}, \mathrm{Fmoc}), 59.2\left(\mathrm{CH}, \mathrm{C}_{2}\right), 59.5\left(\mathrm{CH}, \mathrm{C}_{\alpha}\right), 67.1\left(\mathrm{CH}_{2}, \mathrm{Fmoc}\right), 86.77,117.93,125.0$, 132.6, 133.3 (5C, Pbf), 126.9-139.2 (15CH and 5C, aromatics and Pbf), 156.7 ( CO, Fmoc), 157.7-159.2 ( $\mathrm{C}=\mathrm{NH}$, guanidine), $174.8(\mathrm{CONH})$; ES-MS $\mathrm{m} / z$, $[\mathrm{M}+1]^{+}$calcd for $\mathrm{C}_{50} \mathrm{H}_{58} \mathrm{~N}_{6} \mathrm{O}_{6} \mathrm{~S}$ : 871.77; found 871.77 (100\%); Anal. calcd. for $\mathrm{C}_{50} \mathrm{H}_{58} \mathrm{~N}_{6} \mathrm{O}_{6} \mathrm{~S}$ : C, 68.94; H, 6.71; N, 9.65. Found: C, 69.07; H, 6.82; N, 9.46.

4.4. General procedure for the synthesis of basic amino acid-derived ureas. Method B. Synthesis of ureas $(\boldsymbol{R})-8 \mathbf{a}, \mathbf{b},(S)-8 \mathbf{a}-\mathbf{c},(\boldsymbol{R})-$ and $(S)-13 \mathbf{a}$, and $(\boldsymbol{R})-$ and $(S)-\mathbf{1 4 - 1 6 b}$

Diethyl amine $(0.32 \mathrm{~mL}, 3 \mathrm{mmol})$ was added to a solution of the corresponding Fmocprotected amine 12a-c $(0.3 \mathrm{mmol})$ and the reaction mixture was stirred at $\mathrm{rt}$ for $2 \mathrm{~h}$. Then, the solvent was evaporated to dryness and the residue was dissolved in EtOAc $(50 \mathrm{~mL})$. The solution was successively washed with $\mathrm{H}_{2} \mathrm{O}(20 \mathrm{~mL})$ and brine $(20 \mathrm{~mL})$, dried over $\mathrm{Na}_{2} \mathrm{SO}_{4}$, and evaporated to dryness. The residue was dissolved in dry $\mathrm{CH}_{2} \mathrm{Cl}_{2}(10 \mathrm{~mL})$, cooled at $0^{\circ} \mathrm{C}$. The corresponding isocyanate $(0,3 \mathrm{mmol})$ was added to this solution and the mixture was stirred for $15 \mathrm{~min}-2 \mathrm{~h}$. Then, the solvent was evaporated and the residue was dissolved in EtOAc $(50 \mathrm{~mL})$. This solution was successively washed with $\mathrm{H}_{2} \mathrm{O}(20 \mathrm{~mL})$ and brine (20 $\mathrm{mL}$ ), dried over $\mathrm{Na}_{2} \mathrm{SO}_{4}$, and evaporated to dryness. The residue was purified by flash chromatography, using $20-100 \%$ gradient of EtOAc in hexane as eluant to give the corresponding urea $(\boldsymbol{R})-\mathbf{8 a}, \mathbf{b},(S)-8 \mathbf{a}-\mathbf{c},(\boldsymbol{R})-$ and $(\boldsymbol{S})-\mathbf{1 3 a}$, and $(\boldsymbol{R})-$ and $(\boldsymbol{S})-\mathbf{1 4 - 1 6 b}$.

4.4.1. $\mathrm{N}_{\delta}$-Boc- $\mathrm{N}_{\alpha}-[(2 \mathrm{R})-3-P h e n y l-1-(3-p h e n y l u r e i d o) p r o p a n-2-y l]$-ornithine benzyl amide $[(\boldsymbol{R})-\mathbf{8 a}]$ 
Foam (53 mg, $31 \%$ ); HPLC $t_{\mathrm{R}} 17.45 \mathrm{~min} ;[\alpha]_{\mathrm{D}}{ }^{20}+7.2(c 1, \mathrm{MeOH}) ;{ }^{1} \mathrm{H}$ NMR (400 MHz, $\left.\mathrm{CDCl}_{3}\right) \delta$ 1.10-2.41 [m, 4H, $\beta-\mathrm{H}$ and $\left.\gamma-\mathrm{H}(\mathrm{Orn})\right], 1.40$ (s, 9H, Boc), 2.58-2.76 [m, 2H, 3-H (Pr)], 2.81 [m, 1H, 2-H (Pr)], 2.84-3.02 [m, 5H, 1-H (Pr), $\alpha-\mathrm{H}$ and $\delta-H(O r n)], 4.29$ [dd, 1H, $\mathrm{J}=6$ and $\left.15 \mathrm{~Hz}, \mathrm{CH}_{2}(\mathrm{Bn})\right], 4.41\left[\mathrm{dd}, 1 \mathrm{H}, \mathrm{J}=6\right.$ and $\left.15 \mathrm{~Hz}, \mathrm{CH}_{2}(\mathrm{Bn})\right], 4.62$ (bs, 1H, NH-Boc), 6.89-7.42 (m, 16H, aromatics and $\mathrm{NH}-\mathrm{Bn}), 5.77$ and $7.57(2 \mathrm{bs}, 2 \mathrm{H}, \mathrm{NHC} \mathrm{ONH}) ; \quad{ }^{13} \mathrm{C} \quad \mathrm{NMR}$ $\left(100 \mathrm{MHz}, \mathrm{CDCl}_{3}\right) \delta 26.0\left(\mathrm{CH}_{2}, \mathrm{C}_{\gamma}\right), 28.4\left(3 \mathrm{CH}_{3}, \mathrm{Boc}\right), 30.7\left(\mathrm{CH}_{2}, \mathrm{C}_{\beta}\right), 39.2\left(\mathrm{CH}_{2}, \mathrm{C}_{\delta}\right), 39.4$ $\left(\mathrm{CH}_{2}, \mathrm{C}_{3}\right), 43.2\left(\mathrm{CH}_{2}, \mathrm{Bn}\right), 43.9\left(\mathrm{CH}_{2}, \mathrm{C}_{1}\right), 60.2\left(\mathrm{CH}, \mathrm{C}_{\alpha}\right), 60.4\left(\mathrm{CH}, \mathrm{C}_{2}\right), 79.4(\mathrm{C}, \mathrm{Boc})$, 119.5-139.2 (15CH and $3 \mathrm{C}$, aromatics), 156.2 (HNCONH), 156.4 (CO, Boc), 175.0 (CONH); ES-MS $m / z[\mathrm{M}+1]^{+}$calcd for $\mathrm{C}_{33} \mathrm{H}_{43} \mathrm{~N}_{5} \mathrm{O}_{4}, 574.33$; found 574.27 (100\%); Anal. calcd. for $\mathrm{C}_{33} \mathrm{H}_{43} \mathrm{~N}_{5} \mathrm{O}_{4}$ : C, 69.08; H, 7.55; N, 12.21. Found: C, 69.13; H, 7.62; N, 12.08.

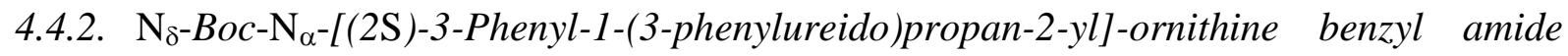
$[(\mathbf{S})-\mathbf{8 a}]$

Foam (144 mg, $84 \%)$; HPLC $t_{\mathrm{R}} 18.15 \mathrm{~min} ;[\alpha]_{\mathrm{D}}{ }^{20}+6.7\left(c\right.$ 1.1, MeOH); ${ }^{1} \mathrm{H}$ NMR (400 $\left.\mathrm{MHz}, \mathrm{CDCl}_{3}\right) \delta 1.10-1.74[\mathrm{~m}, 4 \mathrm{H}, \beta-\mathrm{H}$ and $\gamma-\mathrm{H}(\mathrm{Orn})], 1.42$ (s, 9H, Boc), 2.40-2.75 [m, 2H, 3-H (Pr)], 2.81-3.14 [m, 2H, 1-H (Pr)], 2.93 [m, 1H, 2-H (Pr)], 3.14- 3.48 [m, 3H, $\alpha-\mathrm{H}$ and $\delta-$ $\mathrm{H}(\mathrm{Orn})], 3.70$ [dd, $1 \mathrm{H}, \mathrm{J}=7$ and $\left.15 \mathrm{~Hz}, \mathrm{CH}_{2}(\mathrm{Bn})\right], 4.20$ (dd, $1 \mathrm{H}, \mathrm{J}=5$ and $\left.15 \mathrm{~Hz}, \mathrm{CH}_{2}(\mathrm{Bn})\right]$, 4.94 (bs, 1H, NH-Boc), 6.86-7.47 (m, 15H, aromatics), 6.04 and 8.62 (2bs, 2H, NHCONH), 7.75 (bs, 1H, NH-Bn); $\left.{ }^{13} \mathrm{C} \mathrm{NMR} \mathrm{(100} \mathrm{MHz,} \mathrm{CDCl}_{3}\right) \delta 26.2\left(\mathrm{CH}_{2}, \mathrm{C}_{\gamma}\right), 28.7\left(3 \mathrm{CH}_{3}, \mathrm{Boc}\right), 30.8$ $\left(\mathrm{CH}_{2}, \mathrm{C}_{\beta}\right), 40.2\left(\mathrm{CH}_{2}, \mathrm{C}_{\delta}\right), 40.6\left(\mathrm{CH}_{2}, \mathrm{C}_{3}\right), 42.4\left(\mathrm{CH}_{2}, \mathrm{Bn}\right), 42.9\left(\mathrm{CH}_{2}, \mathrm{C}_{1}\right), 59.5\left(\mathrm{CH}, \mathrm{C}_{2}\right)$, $59.6\left(\mathrm{CH}, \mathrm{C}_{\alpha}\right), 79.9(\mathrm{C}, \mathrm{Boc}), 119.6-139.6$ (15CH and 3C, aromatics), $156.8(\mathrm{HNCONH})$, 156.9 (CO, Boc), 174.7 (CONH); ES-MS $m / z[\mathrm{M}+1]^{+}$calcd for $\mathrm{C}_{33} \mathrm{H}_{43} \mathrm{~N}_{5} \mathrm{O}_{4}, 574.33$; found 574,52 (100\%), $699.43(10 \%)[\mathrm{M}+\mathrm{Na}]^{+}$; Anal. calcd. for $\mathrm{C}_{33} \mathrm{H}_{43} \mathrm{~N}_{5} \mathrm{O}_{4}: \mathrm{C}, 69.08 ; \mathrm{H}, 7.55 ; \mathrm{N}$, 12.21. Found: C, 69.17; H, 7.60; N, 12.13.

4.4.3. $\mathrm{N}_{\varepsilon}-$ Boc- $\mathrm{N}_{\alpha}-[(2 \mathrm{R})-3-P h e n y l-1-(3-p h e n y l u r e i d o)$ propan-2-yl]-lysine benzyl amide $[(\boldsymbol{R})-$ $\mathbf{8 b}]$

Foam (121 mg, 69\%); HPLC $t_{\mathrm{R}} 18.29 \mathrm{~min} ;[\alpha]_{\mathrm{D}}{ }^{20}+6.5(c 0.6, \mathrm{MeOH}) ;{ }^{1} \mathrm{H}$ NMR (400 $\left.\mathrm{MHz}, \mathrm{CDCl}_{3}\right) \delta$ 0.73-1.65 [m, 6H, $\beta-\mathrm{H}, \gamma-\mathrm{H}$ and $\delta-\mathrm{H}$ (Lys)], 1.37 (s, 9H, Boc), 2.53-2.87 [m, 3H, 3-H and 2-H (Pr)], 2.89-3.00 [m, 2H, 1-H (Pr)], 3.01-3.42 [m, 3H, $\alpha-\mathrm{H}$ and $\varepsilon-\mathrm{H}$ (Lys)], 4.29 [dd, $1 \mathrm{H}, \mathrm{J}=6$ and $15 \mathrm{~Hz}, \mathrm{CH}_{2}(\mathrm{Bn})$ ], 4.39 [dd, , $1 \mathrm{H}, \mathrm{J}=6$ and $15 \mathrm{~Hz}, \mathrm{CH}_{2}(\mathrm{Bn})$ ], 4.59 (s, 1H, $N H$-Boc), 5.51 (bs, 1H, HNCONH), 6.87-7.34 (m, 16H, aromatics and HNCONH), 7.56 (bs, $1 \mathrm{H}, \mathrm{NH}-\mathrm{Bn}) ;{ }^{13} \mathrm{C} \mathrm{NMR}\left(100 \mathrm{MHz}, \mathrm{CDCl}_{3}\right) \delta 22.6\left(\mathrm{CH}_{2}, \mathrm{C}_{\gamma}\right), 28.4\left(3 \mathrm{CH}_{3}, \mathrm{Boc}\right), 29.6$ 
$\left(\mathrm{CH}_{2}, \mathrm{C}_{\delta}\right), 33.3\left(\mathrm{CH}_{2}, \mathrm{C}_{\beta}\right), 39.0\left(\mathrm{CH}_{2}, \mathrm{C}_{3}\right), 39.9\left(\mathrm{CH}_{2}, \mathrm{C}_{\varepsilon}\right), 43.1\left(\mathrm{CH}_{2}, \mathrm{Bn}\right), 43.8\left(\mathrm{CH}_{2}, \mathrm{C}_{1}\right)$, $59.1\left(\mathrm{CH}, \mathrm{C}_{2}\right),\left(61.0\left(\mathrm{CH}, \mathrm{C}_{\alpha}\right), 79.3(\mathrm{C}, \mathrm{Boc}), 122.8-138.4(15 \mathrm{CH}\right.$ and $3 \mathrm{C}$, aromatics), 156.0 (HNCONH), $156.2(\mathrm{CO}, \mathrm{Boc}), 174.0(\mathrm{CONH})$; ES-MS $\mathrm{m} / z[\mathrm{M}+1]^{+}$calcd for $\mathrm{C}_{34} \mathrm{H}_{45} \mathrm{~N}_{5} \mathrm{O}_{4}$, 588.35; found 588.62 (100\%); Anal. calcd. for $\mathrm{C}_{34} \mathrm{H}_{45} \mathrm{~N}_{5} \mathrm{O}_{4}$ : C, 69.48; H, 7.72; N, 11.92 . Found: C, 69.62; H, 7.89; N, 12.75 .

4.4.4. $\mathrm{N}_{\varepsilon}-$ Boc- $\mathrm{N}_{\alpha}-[(2 \mathrm{~S})-3-P h e n y l-1-(3-p h e n y l u r e i d o)$ propan-2-yl]-lysine benzyl amide $[(\boldsymbol{S})$ 8b]

Foam $(143 \mathrm{mg}, 81 \%)$; HPLC $t_{\mathrm{R}} 18.49 \mathrm{~min} ;[\alpha]_{\mathrm{D}}{ }^{20}+4.0(c 0.9, \mathrm{MeOH}) ;{ }^{1} \mathrm{H}$ NMR (400 $\left.\mathrm{MHz}, \mathrm{CDCl}_{3}\right) \delta 1.00-1.90[\mathrm{~m}, 6 \mathrm{H}, \beta-\mathrm{H}, \gamma-\mathrm{H}$ and $\delta-\mathrm{H}$ (Lys)], 1.46 (s, 9H, Boc), 2.51-2.68 [m, 2H, 3-H (Pr)], 2.73 [m, 1H, 2-H (Pr)], 2.90-3.18 [m, 2H, ع-H (Lys)], 3.18-3.50 [m, 3H, 1-H (Pr) and $\alpha-\mathrm{H}(\mathrm{Lys})], 3.90\left[\mathrm{dd}, 1 \mathrm{H}, \mathrm{J}=7\right.$ and $\left.15 \mathrm{~Hz}, \mathrm{CH}_{2}(\mathrm{Bn})\right], 4.35$ [dd, $1 \mathrm{H}, \mathrm{J}=4$ and $15 \mathrm{~Hz}$, $\left.\mathrm{CH}_{2}(\mathrm{Bn})\right], 4.88$ (bs, $1 \mathrm{H}, \mathrm{NH}$-Boc), 6.82-7.20 (m,16H, aromatics and $\left.N H-\mathrm{Bn}\right), 5.98$ and 8.09 (2bs, 2H, HNCONH); ${ }^{13} \mathrm{C}$ NMR (100 MHz, $\left.\mathrm{CDCl}_{3}\right) \delta 21.1\left(\mathrm{CH}_{2}, \mathrm{C}_{\gamma}\right), 26.3\left(3 \mathrm{CH}_{3}, \mathrm{Boc}\right), 27.6$ $\left(\mathrm{CH}_{2}, \mathrm{C}_{\delta}\right), 27.9\left(\mathrm{CH}_{2}, \mathrm{C}_{\beta}\right), 30.9\left(\mathrm{CH}_{2}, \mathrm{C}_{3}\right), 38.1\left(\mathrm{CH}_{2}, \mathrm{C}_{\varepsilon}\right), 39.8\left(\mathrm{CH}_{2}, \mathrm{C}_{1}\right), 40.7\left(\mathrm{CH}_{2}, \mathrm{Bn}\right)$, $58.0\left(\mathrm{CH}, \mathrm{C}_{2}\right), 58.4\left(\mathrm{CH}, \mathrm{C}_{\alpha}\right), 77.4(\mathrm{C}, \mathrm{Boc}), 117.0-137.3(15 \mathrm{CH}$ and $3 \mathrm{C}$, aromatics), 154.7 (CO, Boc), $154.8(\mathrm{HNCONH}), 172.1(\mathrm{CONH})$; ES-MS $\mathrm{m} / z[\mathrm{M}+1]^{+}$calcd for $\mathrm{C}_{34} \mathrm{H}_{45} \mathrm{~N}_{5} \mathrm{O}_{4}$, 588.35; found, 588.55 (100\%); Anal. calcd. for $\mathrm{C}_{34} \mathrm{H}_{45} \mathrm{~N}_{5} \mathrm{O}_{4}$ : C, 69.48; H, 7.72; N, 11.92 . Found: C, 69.59; H, 7.84; N, 12.85 .

4.4.5. $\mathrm{N}_{\varepsilon}-P b f-\mathrm{N}_{\alpha}-[(2 \mathrm{~S})-3-P h e n y l-1-(3-p h e n y l u r e i d o) p r o p a n-2-y l]$-arginine benzyl amide $[(S)-8 c]$

Foam ( $68 \mathrm{mg}, 29 \%$ ); HPLC $t_{\mathrm{R}} 19.95 \mathrm{~min} ;[\alpha]_{\mathrm{D}}{ }^{20}+2.5$ (c 1, MeOH); ${ }^{1} \mathrm{H}-\mathrm{NMR}(500 \mathrm{MHz}$, $\left.\mathrm{CDCl}_{3}\right) \delta$ 0.61-1.63 [m, 4H, $\beta$ - and $\left.\gamma-\mathrm{H}(\mathrm{Arg})\right], 1.38,2.01,2.39$ and $2.45\left[4 \mathrm{~s}, 15 \mathrm{H}, \mathrm{CH}_{3}\right.$ $(\mathrm{Pbf})], 2.74[\mathrm{~m}, 1 \mathrm{H}, 2-\mathrm{H}(\mathrm{Pr})], 2.85$ [s, 2H, $\left.\mathrm{CH}_{2}(\mathrm{Pbf})\right], 2.92-3.17$ [m, 4H, 3-H (Pr) and $\delta-$ $\mathrm{H}(\mathrm{Arg})], 3.20-3.42$ [m, 2H, 1-H (Pr)], 3.99 [bs, 1H, $\alpha-\mathrm{H}(\mathrm{Arg})], 4.17$ [d, 1H, J = 6 and 14.5 $\mathrm{Hz}, \mathrm{CH}_{2}(\mathrm{Bn})$ ], 4.30 [dd, $1 \mathrm{H}, \mathrm{J}=4.5$ and $14.5 \mathrm{~Hz}, \mathrm{CH}_{2}(\mathrm{Bn})$ ], 6.20 [bs, 3H, guanidino], 6.85 (t, $1 \mathrm{H}, \mathrm{J}=6 \mathrm{~Hz}, \mathrm{NH}-\mathrm{Bn}), 7.00-7.34$ (m, 15H, aromatics), 7.78 and 8.15 (2bs, 2H, NHCONH); ${ }^{13} \mathrm{C}-\mathrm{NMR}\left(125 \mathrm{MHz}, \mathrm{CDCl}_{3}\right) \delta 12.9,18.3,19.7,28.9\left(\mathrm{CH}_{3}, \mathrm{Pbf}\right), 25.7\left(\mathrm{CH}_{2}, \mathrm{C}_{\gamma}\right), 30.1\left(\mathrm{CH}_{2}\right.$, $\left.\mathrm{C}_{\beta}\right), 31.3\left(\mathrm{CH}_{2}, \mathrm{C}_{3}\right), 40.3\left(\mathrm{CH}_{2}, \mathrm{C}_{\delta}\right), 43.6\left(\mathrm{CH}_{2}, \mathrm{C}_{1}\right), 43.6\left(\mathrm{CH}_{2}, \mathrm{Pbf}\right), 59.2\left(\mathrm{CH}, \mathrm{C}_{2}\right), 59.5(\mathrm{CH}$, $\left.\mathrm{C}_{\alpha}\right), 86.77,117.93,125.0,132.6,133.3$ (5 C, Pbf), 126.9-139.2 [15 CH and 4C, aromatics and Pbf], $156.4\left(\mathrm{HNCONH}\right.$ ), 159.2 (guanidino); ES-MS $\mathrm{m} / z[\mathrm{M}+1]^{+}$calcd. for $\mathrm{C}_{42} \mathrm{H}_{53} \mathrm{~N}_{7} \mathrm{O}_{5} \mathrm{~S}$, 
767.38; found, 768.81 (100\%). Anal. calcd. for $\mathrm{C}_{42} \mathrm{H}_{53} \mathrm{~N}_{7} \mathrm{O}_{5} \mathrm{~S}: \mathrm{C}, 65.69 ; \mathrm{H}, 6.96 ; \mathrm{N}, 12.77$; Found: C, 65.82; H, 7.04; N, 12.68.

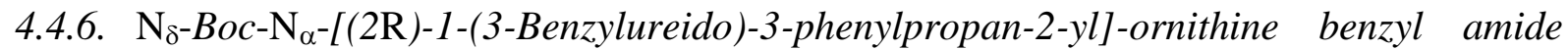
$[(\boldsymbol{R})-\mathbf{1 3 a}]$

Foam (80 mg, $45 \%)$; HPLC $t_{\mathrm{R}} 18.47 \mathrm{~min} ;[\alpha]_{\mathrm{D}}{ }^{20}+6.5\left(c\right.$ 1.1, MeOH); ${ }^{1} \mathrm{H}$ NMR $(500 \mathrm{MHz}$, $\left.\mathrm{CDCl}_{3}\right) \delta$ 1.19-1.69 [m, 4H, $\beta-\mathrm{H}$ and $\left.\gamma-\mathrm{H}(\mathrm{Orn})\right], 1.39$ (s, 9H, Boc), 2.61-2.65 [m, 2H, 3-H (Pr)], $2.80[\mathrm{~m}, 1 \mathrm{H}, 2-\mathrm{H}(\operatorname{Pr})], 2.87-3.20[\mathrm{~m}, 4 \mathrm{H}, 1-\mathrm{H}(\operatorname{Pr})$, and $\delta-\mathrm{H}(\mathrm{Orn})], 3.21[\mathrm{~m}, 1 \mathrm{H}, \alpha-\mathrm{H}$ (Orn)], 4.27 [m, 2H, $\mathrm{CH}_{2}$ (Bn-ureido)], 4.32 [dd, 1H, J= 6 and $15 \mathrm{~Hz}, \mathrm{CH}_{2}$ (Bn)], 4.40 [ dd, $1 \mathrm{H}, \mathrm{J}=6$ and $\left.15 \mathrm{~Hz}, \mathrm{CH}_{2}(\mathrm{Bn})\right], 4.58$ (bs, $1 \mathrm{H}, \mathrm{NH}$-Boc), 4.73 and 5.06 (2bs, 2H, NHCONH), 7.07-7.36 (m, 16H, aromatics and $\mathrm{NH}-\mathrm{Bn}) ;{ }^{13} \mathrm{C} \mathrm{NMR}\left(100 \mathrm{MHz}, \mathrm{CDCl}_{3}\right) \delta 26.1\left(\mathrm{CH}_{2}, \mathrm{C}_{\gamma}\right)$, $28.4\left(3 \mathrm{CH}_{3}, \mathrm{Boc}\right), 30.6\left(\mathrm{CH}_{2}, \mathrm{C}_{\beta}\right), 39.4\left(\mathrm{CH}_{2}, \mathrm{C}_{3}\right), 39.5\left(\mathrm{CH}_{2}, \mathrm{C}_{\delta}\right), 43.2\left(\mathrm{CH}_{2}, \mathrm{Bn}\right), 43.9\left[\mathrm{CH}_{2}\right.$, (Bn-ureido)], $44.4\left(\mathrm{CH}_{2}, \mathrm{C}_{1}\right), 59.4\left(\mathrm{CH}, \mathrm{C}_{2}\right), 59.9\left(\mathrm{CH}, \mathrm{C}_{\alpha}\right), 79.4(\mathrm{C}, \mathrm{Boc}), 127.2-138.7$ (15CH and $3 \mathrm{C}$, aromatics), 156.2 (CO, Boc), 158.2 (HNCONH), $174.6(\mathrm{CONH})$; ES-MS $m / z[\mathrm{M}+1]^{+}$calcd for $\mathrm{C}_{34} \mathrm{H}_{45} \mathrm{~N}_{5} \mathrm{O}_{4}, 588.35$; found 588.48 (100\%); Anal. calcd. for $\mathrm{C}_{34} \mathrm{H}_{45} \mathrm{~N}_{5} \mathrm{O}_{4}$ : C, 69.48; H, 7.72; N, 11.92. Found: C, 69.61; H, 7.62; N, 12.08.

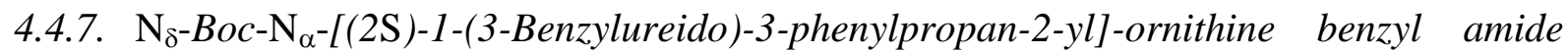
$[(S)-13 a]$

Foam (112 mg, $64 \%)$; HPLC $t_{\mathrm{R}} 18.86 \mathrm{~min} ;[\alpha]_{\mathrm{D}}{ }^{20}+7.0(c 0.9, \mathrm{MeOH}) ;{ }^{1} \mathrm{H}$ NMR (400 $\left.\mathrm{MHz}, \mathrm{CDCl}_{3}\right) \delta 1.31$ (s, 9H, Boc), 1.41-1.85 [m, 4H, $\beta-\mathrm{H}$ and $\left.\gamma-\mathrm{H}(\mathrm{Orn})\right], 2.45-2.81[\mathrm{~m}, 2 \mathrm{H}$, 3-H (Pr)], 2.74 [m, 1H, 2-H (Pr)], 2.86-3.34 [m, 4H, 1-H (Pr) and $\delta-H(O r n)], 3.42$ [m, 2H, $\alpha-\mathrm{H}(\mathrm{Orn})], 3.78$ [dd, 1H, J=7 and 15Hz, $\left.\mathrm{CH}_{2}(\mathrm{Bn})\right], 4.30$ (dd, $1 \mathrm{H}, \mathrm{J}=4$ and $\left.15 \mathrm{~Hz}, \mathrm{CH}_{2}(\mathrm{Bn})\right]$, 4.37 [m, 2H, $\mathrm{CH}_{2}$ (Bn-ureido)], 4.94 (bs, 1H, NH-Boc), 6.86-7.47 (m, 15H, aromatics), 5.81 (1bs, 2H, NHCONH), 6.96 (bs, $1 \mathrm{H}, \mathrm{NH}$-Bn); ${ }^{13} \mathrm{C} \mathrm{NMR}\left(100 \mathrm{MHz}, \mathrm{CDCl}_{3}\right) \delta 25.8\left(\mathrm{CH}_{2}, \mathrm{C}_{\gamma}\right)$, $28.3\left(3 \mathrm{CH}_{3}, \mathrm{Boc}\right), 30.5\left(\mathrm{CH}_{2}, \mathrm{C}_{\beta}\right), 39.8\left(\mathrm{CH}_{2}, \mathrm{C}_{\delta}\right), 40.3\left(\mathrm{CH}_{2}, \mathrm{C}_{3}\right), 41.7\left(\mathrm{CH}_{2}, \mathrm{Bn}\right), 42.6\left[\mathrm{CH}_{2}\right.$, (Bn-ureido)], $44.3\left(\mathrm{CH}_{2}, \mathrm{C}_{1}\right), 58.6\left(\mathrm{CH}, \mathrm{C}_{2}\right), 59.2\left(\mathrm{CH}, \mathrm{C}_{\alpha}\right), 79.7(\mathrm{C}, \mathrm{Boc}), 126.3-139.7$ (15CH and 3C, aromatics), $156.7(\mathrm{CO}, \mathrm{Boc}), 159.2(\mathrm{HNCONH}), 174.4(\mathrm{CONH}) ; \mathrm{ES}-\mathrm{MS} \mathrm{m} / \mathrm{z}$ $[\mathrm{M}+1]^{+}$calcd for $\mathrm{C}_{34} \mathrm{H}_{45} \mathrm{~N}_{5} \mathrm{O}_{4}, 588.35$; found 588.62 (100\%); Anal. calcd. for $\mathrm{C}_{34} \mathrm{H}_{45} \mathrm{~N}_{5} \mathrm{O}_{4}$ : $\mathrm{C}$, 69.48; H, 7.72; N, 11.92. Found: C, 69.63; H, 7.84; N, 11.87.

4.4.8. $\quad \mathrm{N}_{\varepsilon}$-Boc- $\mathrm{N}_{\alpha}-[(2 \mathrm{R})-1-(3-(2,4-D i c h l o r o p h e n y l)$ ureido)-3-phenylpropan-2-yl]-lysine benzyl amide $[(\boldsymbol{R})-\mathbf{1 4 b}]$ 
Foam (125 mg, 63\%); HPLC $t_{\mathrm{R}} 20.05 \mathrm{~min} ;[\alpha]_{\mathrm{D}}{ }^{20}+7.5(c 1, \mathrm{MeOH}) ;{ }^{1} \mathrm{H}$ NMR $(400 \mathrm{MHz}$, $\left.\mathrm{CDCl}_{3}\right) \delta$ 0.73-1.65 [m, 6H, $\beta-\mathrm{H}, \gamma-\mathrm{H}$ and $\delta-\mathrm{H}$ (Lys)], 1.42 (s, 9H, Boc), 2.63 [dd, 1H, J= 6 and $13.5 \mathrm{~Hz}, 3-\mathrm{H}(\mathrm{Pr})], 2.72$ [dd, 1H, J= 6 and $13.5 \mathrm{~Hz}, 3-\mathrm{H}(\mathrm{Pr})], 2.83-3.14$ [m, 5H, 1-H (Pr), 2-H (Pr), and $\varepsilon-\mathrm{H}(\mathrm{Lys})], 3.32$ [m, 1H, $\alpha-\mathrm{H}(\mathrm{Lys})], 4.30$ [dd, 1H, J= 6 and $\left.15 \mathrm{~Hz}, \mathrm{CH}_{2}(\mathrm{Bn})\right]$, 4.40 [dd, , 1H, J= 6 and $\left.15 \mathrm{~Hz}, \mathrm{CH}_{2}(\mathrm{Bn})\right], 4.55$ (s, 1H, NH-Boc), 5.96 (bs, 2H, HNCONH), 7.04-7.31 (m, 13H, aromatics), 7.33 (bs, 1H, NH-Bn); $\left.{ }^{13} \mathrm{C} \mathrm{NMR} \mathrm{(100} \mathrm{MHz,} \mathrm{CDCl}_{3}\right) \delta 22.5$ $\left(\mathrm{CH}_{2}, \mathrm{C}_{\gamma}\right), 28.4\left(3 \mathrm{CH}_{3}, \mathrm{Boc}\right), 29.6\left(\mathrm{CH}_{2}, \mathrm{C}_{\delta}\right), 33.6\left(\mathrm{CH}_{2}, \mathrm{C}_{\beta}\right), 39.2\left(\mathrm{CH}_{2}, \mathrm{C}_{3}\right), 39.8\left(\mathrm{CH}_{2}, \mathrm{C}_{\varepsilon}\right)$, $43.2\left(\mathrm{CH}_{2}, \mathrm{Bn}\right), 43.9\left(\mathrm{CH}_{2}, \mathrm{C}_{1}\right), 58.9\left(\mathrm{CH}, \mathrm{C}_{2}\right), 60.8\left(\mathrm{CH}, \mathrm{C}_{\alpha}\right), 79.4(\mathrm{C}, \mathrm{Boc}), 121.5-138.2$ (13CH and 5C, aromatics), 154.9 (HNCONH), 156.3 (CO, Boc), $175.0(\mathrm{CONH}) ; \mathrm{ES}-\mathrm{MS} \mathrm{m} / \mathrm{z}$ $[\mathrm{M}+1]^{+}$calcd for $\mathrm{C}_{34} \mathrm{H}_{43} \mathrm{Cl}_{2} \mathrm{~N}_{5} \mathrm{O}_{4}, 656.27$; found, 656.66 (100\%), 658.62 (70\%) $[\mathrm{M}+3]^{+}$, $660.65(10 \%)[\mathrm{M}+5]^{+}$; Anal. calcd. for $\mathrm{C}_{34} \mathrm{H}_{43} \mathrm{Cl}_{2} \mathrm{~N}_{5} \mathrm{O}_{4}$ : C, 62.19; H, 6.60; N, 10.67. Found: C, 62.32; H, 6.75; N, 10.48 .

4.4.9. $\mathrm{N}_{\varepsilon}$-Boc- $\mathrm{N}_{\alpha-}[(2 \mathrm{~S})-1-(3-(2,4-D i c h l o r o p h e n y l) u r e i d o)-3-p h e n y l p r o p a n-2-y l]-l y s i n e$ benzyl amide $[(\boldsymbol{S})-\mathbf{1 4 b}]$

Foam (147 mg, 74\%); HPLC $t_{\mathrm{R}} 20.03 \mathrm{~min} ;[\alpha]_{\mathrm{D}}{ }^{20}+3.0(c 0.8, \mathrm{MeOH}) ;{ }^{1} \mathrm{H}$ NMR (300 $\left.\mathrm{MHz}, \mathrm{CDCl}_{3}\right) \delta$ 0.86-2.19 [m, 6H, $\beta-\mathrm{H}, \gamma-\mathrm{H}$ and $\delta-\mathrm{H}$ (Lys)], 1.45 (s, 9H, Boc), 2.66-2.91 [m, 3H, 2-H and 3-H (Pr)], 3.00-3.54 [m, 5H, 1-H (Pr), $\alpha-\mathrm{H}$ and $\varepsilon-\mathrm{H}(\mathrm{Lys})$ ], 4.00 [dd, 1H, J= 6.5 and $15 \mathrm{~Hz}, \mathrm{CH}_{2}(\mathrm{Bn})$ ], 4.37 [dd, $, 1 \mathrm{H}, \mathrm{J}=6.5$ and $15 \mathrm{~Hz}, \mathrm{CH}_{2}(\mathrm{Bn})$ ], 4.86 (s, 1H, NH-Boc), 6.33 (bs, 2H, HNCONH), 6.90-7.58 (m, 13H, aromatics), 7.70 (bs, $1 \mathrm{H}, N H-\mathrm{Bn}) ;{ }^{13} \mathrm{C}$ NMR $\left(100 \mathrm{MHz}, \mathrm{CDCl}_{3}\right) \delta 20.8\left(\mathrm{CH}_{2}, \mathrm{C}_{\gamma}\right), 28.5\left(3 \mathrm{CH}_{3}, \mathrm{Boc}\right), 29.7\left(\mathrm{CH}_{2}, \mathrm{C}_{\beta}\right.$ and $\left.\mathrm{C}_{\delta}\right), 36.6\left(\mathrm{CH}_{2}\right.$, $\left.\mathrm{C}_{3}\right), 39.7\left(\mathrm{CH}_{2}, \mathrm{C}_{\varepsilon}\right), 40.0\left(\mathrm{CH}_{2}, \mathrm{Bn}\right), 42.9\left(\mathrm{CH}_{2}, \mathrm{C}_{1}\right), 60.2\left(\mathrm{CH}, \mathrm{C}_{2}\right), 60.4\left(\mathrm{CH}, \mathrm{C}_{\alpha}\right), 79.9(\mathrm{C}$, Boc), 121.4-138.5 (13CH and 5C, aromatics), 154.7 (CO, Boc),157.2 (HNCONH), 170.2 $(\mathrm{CONH})$; ES-MS $\mathrm{m} / \mathrm{z}[\mathrm{M}+1]^{+}$calcd for $\mathrm{C}_{34} \mathrm{H}_{43} \mathrm{Cl}_{2} \mathrm{~N}_{5} \mathrm{O}_{4}, 656.27$; found, $656.46(100 \%)$, $658.48(80 \%)[\mathrm{M}+3]^{+}, 660.49(15 \%)[\mathrm{M}+5]^{+}$; Anal. calcd. for $\mathrm{C}_{34} \mathrm{H}_{43} \mathrm{Cl}_{2} \mathrm{~N}_{5} \mathrm{O}_{4}: \mathrm{C}, 62.19 ; \mathrm{H}$, $6.60 ; \mathrm{N}, 10.67$. Found: C, 62.29; H, 6.71; N, 10.55 .

4.4.10. $\mathrm{N}_{\varepsilon}$-Boc- $\mathrm{N}_{\alpha}-[(2 \mathrm{R})-1-(3-(4-M e t h o x y p h e n e t h y l)$ ureido)-3-phenylpropan-2-yl]-lysine benzyl amide $[(\boldsymbol{R})-\mathbf{1 5 b}]$

Foam $(127 \mathrm{mg}, 66 \%)$; HPLC $t_{\mathrm{R}} 18.29 \mathrm{~min} ;[\alpha]_{\mathrm{D}}{ }^{20}+8.5(c 1.1, \mathrm{MeOH}) ;{ }^{1} \mathrm{H}$ NMR (400 $\left.\mathrm{MHz}, \mathrm{CDCl}_{3}\right) \delta$ 0.78-1.71 [m, 6H, $\beta-\mathrm{H}, \gamma-\mathrm{H}$ and $\delta-\mathrm{H}$ (Lys)], 1.42 (s, 9H, Boc), 2.66 [t, 2H, J= $7 \mathrm{~Hz}, 2-\mathrm{H}$ (ethyl)], 2.66-2.82 [m, 3H, 2-H and 3-H (Pr)], 2.91-3-18 [m, 5H, 1-H (Pr), $\alpha-\mathrm{H}$ and $\varepsilon-\mathrm{H}$ (Lys)], 3.28 [dd, 2H, J= 7 and 13 Hz, 1-H (ethyl)], 3.76 (s, 3H, OMe), 4.32 [dd, 1H, J=6 
and $14.5 \mathrm{~Hz}, \mathrm{CH}_{2}(\mathrm{Bn})$ ], 4.41 [dd, , 1H, J= 6 and $\left.14.5 \mathrm{~Hz}, \mathrm{CH}_{2}(\mathrm{Bn})\right], 4.55$ (bs, 1H, NH-Boc), 4.70 (bs, 2H, HNCONH), 7.04-7.33 (m, 14H, aromatics), 7.57 (bs, $1 \mathrm{H}, N H-\mathrm{Bn}) ;{ }^{13} \mathrm{C} \mathrm{NMR}$ $\left(100 \mathrm{MHz}, \mathrm{CDCl}_{3}\right) \delta 22.6\left(\mathrm{CH}_{2}, \mathrm{C}_{\gamma}\right), 28.4\left(3 \mathrm{CH}_{3}, \mathrm{Boc}\right), 29.7\left(\mathrm{CH}_{2}, \mathrm{C}_{\delta}\right), 30.0\left(\mathrm{CH}_{2}, \mathrm{C}_{\beta}\right), 35.4$ $\left(\mathrm{CH}_{2}, \mathrm{C}_{3}\right), 35.5\left[\mathrm{CH}_{2}, \mathrm{C}_{2}\right.$ (ethyl)], $38.9\left[\mathrm{CH}_{2}, \mathrm{C}_{\varepsilon}\right.$ and $\mathrm{C}_{1}$ (ethyl)], $43.1\left(\mathrm{CH}_{2}, \mathrm{Bn}\right), 43.9\left(\mathrm{CH}_{2}\right.$, $\left.\mathrm{C}_{1}\right), 55.2\left(\mathrm{CH}_{3}, \mathrm{OMe}\right), 59.5\left(\mathrm{CH}, \mathrm{C}_{2}\right), 60.9\left(\mathrm{CH}, \mathrm{C}_{\alpha}\right), 79.1(\mathrm{C}, \mathrm{Boc}), 113.9-138.5(14 \mathrm{CH}$ and 4C, aromatics), 156.0 (CO, Boc), $158.3(\mathrm{HNCONH}), 174.6(\mathrm{CONH})$; ES-MS m/z $[\mathrm{M}+1]^{+}$ calcd for $\mathrm{C}_{37} \mathrm{H}_{51} \mathrm{~N}_{5} \mathrm{O}_{5}$, 646.39; found, 646.72 (100\%); Anal. calcd. for $\mathrm{C}_{37} \mathrm{H}_{51} \mathrm{~N}_{5} \mathrm{O}_{5}$ : C, 68.81; H, 7.96; N, 10.84. Found: C, 68.96; H, 8.07; N, 10.48 .

\subsubsection{1. $\mathrm{N}_{\varepsilon}-$ Boc- $\mathrm{N}_{\alpha}-[(2 \mathrm{~S})-1-(3-(4-M e t h o x y p h e n e t h y l)$ ureido)-3-phenylpropan-2-yl]-lysine benzyl amide $[(\boldsymbol{S})-\mathbf{1 5 b}]$}

Foam (143 mg, 87\%); HPLC $t_{\mathrm{R}} 18.43 \mathrm{~min} ;[\alpha]_{\mathrm{D}}{ }^{20}+5.9(c 1, \mathrm{MeOH}) ;{ }^{1} \mathrm{H}$ NMR $(300 \mathrm{MHz}$, $\left.\mathrm{CDCl}_{3}\right) \delta$ 1.09-2.23 [m, 6H, $\beta-\mathrm{H}, \gamma-\mathrm{H}$ and $\delta$-H (Lys)], 1.43 (s, 9H, Boc), 2.60-2.69 [m, 2H, 3H (Pr)], 2.65-2.72 [m, 2H, 2-H (Pr)], 2.76 [t, 2H, J= 7 Hz, 2-H (ethyl)], 2.99-3-17 [m, 4H, 1$\mathrm{H}(\mathrm{Pr})$ and $\varepsilon-\mathrm{H}(\mathrm{Lys})], 3.29-3.50[\mathrm{~m}, 1 \mathrm{H}, \alpha-\mathrm{H}(\mathrm{Lys})], 3.40$ [dd, 2H, J= 7 and $13 \mathrm{~Hz}, 1-\mathrm{H}$ (ethyl)], 3.80 (s, 3H, OMe), 3.91 [dd, 1H, J= 7 and $15 \mathrm{~Hz}, \mathrm{CH}_{2}(\mathrm{Bn})$ ], 4.35 [dd, , 1H, J= 7 and $15 \mathrm{~Hz}, \mathrm{CH}_{2}(\mathrm{Bn})$ ], 4.85 (bs, 1H, NH-Boc), 5.15 and 5.29 (2bs, 2H, HNCONH), 6.75-7.44 (m, $15 \mathrm{H}$, aromatics and $\mathrm{NH}-\mathrm{Bn}) ;{ }^{13} \mathrm{C} \mathrm{NMR}\left(75 \mathrm{MHz}, \mathrm{CDCl}_{3}\right) \delta 23.6\left(\mathrm{CH}_{2}, \mathrm{C}_{\gamma}\right), 28.8\left(3 \mathrm{CH}_{3}, \mathrm{Boc}\right)$, $30.1\left(\mathrm{CH}_{2}, \mathrm{C}_{\delta}\right), 30.5\left(\mathrm{CH}_{2}, \mathrm{C}_{\beta}\right), 33.5\left(\mathrm{CH}_{2}, \mathrm{C}_{3}\right), 35.5\left[\mathrm{CH}_{2}, \mathrm{C}_{2}\right.$ (ethyl)], $40.5\left(\mathrm{CH}_{2}, \mathrm{C}_{\varepsilon}\right), 40.8$ $\left[\mathrm{CH}_{2}, \mathrm{C}_{1}\right.$ (ethyl) $], 42.2\left(\mathrm{CH}_{2}, \mathrm{Bn}\right), 43.1\left(\mathrm{CH}_{2}, \mathrm{C}_{1}\right), 55.6\left(\mathrm{CH}_{3}, \mathrm{OMe}\right), 60.6\left(\mathrm{CH}, \mathrm{C}_{2}\right), 60.8(\mathrm{CH}$, $\left.\mathrm{C}_{\alpha}\right), 79.9$ (C, Boc), 114.3-139.5 (14CH and 4C, aromatics), 157.1 (CO, Boc), 159.5 (HNCONH), $174.9(\mathrm{CONH})$; ES-MS $\mathrm{m} / \mathrm{z}[\mathrm{M}+1]^{+}$calcd for $\mathrm{C}_{37} \mathrm{H}_{51} \mathrm{~N}_{5} \mathrm{O}_{5}, 646.39$; found, 646.52 (100\%); Anal. calcd. for $\mathrm{C}_{37} \mathrm{H}_{51} \mathrm{~N}_{5} \mathrm{O}_{5}$ : C, 68.81; H, 7.96; N, 10.84. Found: C, 68.93; H, 8.01; N, 10.69 .

4.4.12. $\mathrm{N}_{\varepsilon}$-Boc- $\mathrm{N}_{\alpha}-[(2 \mathrm{R})-1-(3-(4-F l u o r o p h e n e t h y l)$ ureido)-3-phenyl)propan-2-yl]-lysine benzyl amide $[(\boldsymbol{R})-\mathbf{1 6 b}]$

Foam $(118 \mathrm{mg}, 63 \%)$; HPLC $t_{\mathrm{R}} 18.47 \mathrm{~min} ;[\alpha]_{\mathrm{D}}{ }^{20}+7.6(c 1, \mathrm{MeOH}) ;{ }^{1} \mathrm{H}$ NMR $(300 \mathrm{MHz}$, $\left.\mathrm{CDCl}_{3}\right) \delta$ 0.81-2.11 [m, 6H, $\beta-\mathrm{H}, \gamma-\mathrm{H}$ and $\delta$-H (Lys)], 1.46 (s, 9H, Boc), 2.56-2.89 [m, 3H, 2$\mathrm{H}$ and 3-H (Pr)], 2.77 [t, 2H, J= 7 Hz, 2-H (ethyl)], 2.93-3.28 [m, 5H, 1-H (Pr), $\alpha-\mathrm{H}$ and $\varepsilon-\mathrm{H}$ (Lys)], 3.32 [dd, 2H, J= 7 and $13 \mathrm{~Hz}, 1-\mathrm{H}$ (ethyl)], 4.41 [d, 2H, J= $7 \mathrm{~Hz}, \mathrm{CH}_{2}$ (Bn)], 4.58 (bs, 1H, $\mathrm{NH}$-Boc), 4.80 (bs, 2H, HNCONH), 6.89-7.48 (m, 14H, aromatics), 7.52 (bs, 1H, $\mathrm{NH}$ $\mathrm{Bn}) ;{ }^{13} \mathrm{C}$ NMR $\left(75 \mathrm{MHz}, \mathrm{CDCl}_{3}\right) \delta 22.3\left(\mathrm{CH}_{2}, \mathrm{C}_{\gamma}\right), 28.0\left(3 \mathrm{CH}_{3}, \mathrm{Boc}\right), 29.3\left(\mathrm{CH}_{2}, \mathrm{C}_{\delta}\right), 30.5$ 
$\left(\mathrm{CH}_{2}, \mathrm{C}_{\beta}\right), 32.9\left[\mathrm{CH}_{2}, \mathrm{C}_{2}\right.$ (ethyl)], $35.2\left(\mathrm{CH}_{2}, \mathrm{C}_{3}\right), 38.9\left(\mathrm{CH}_{2}, \mathrm{C}_{\varepsilon}\right), 41.2\left[\mathrm{CH}_{2}, \mathrm{C}_{1}\right.$ (ethyl)], 42.7 $\left(\mathrm{CH}_{2}, \mathrm{Bn}\right), 43.8\left(\mathrm{CH}_{2}, \mathrm{C}_{1}\right), 59.1\left(\mathrm{CH}, \mathrm{C}_{2}\right), 60.7\left(\mathrm{CH}, \mathrm{C}_{\alpha}\right), 79.8(\mathrm{C}, \mathrm{Boc}), 114.7-159.5(14 \mathrm{CH}$ and 4C, aromatics), 155.7 (CO, Boc), $157.9(\mathrm{HNCONH}), 174.4(\mathrm{CONH})$; ES-MS $m / z[\mathrm{M}+1]^{+}$ calcd for $\mathrm{C}_{36} \mathrm{H}_{48} \mathrm{FN}_{5} \mathrm{O}_{4}$, 634.37; found, 634.68 (100\%); Anal. calcd. for $\mathrm{C}_{36} \mathrm{H}_{48} \mathrm{FN}_{5} \mathrm{O}_{4}$ : C, 68.22; H, 7.63; N, 11.05. Found: C, 68.38; H, 7.75; N, 10.89 .

4.4.13. $\mathrm{N}_{\varepsilon}$-Boc- $\mathrm{N}_{\alpha}-[(2 \mathrm{~S})-1-(3-(4-F l u o r o p h e n e t h y l)$ ureido)-3-phenyl)propan-2-yl]-lysine benzyl amide $[(\boldsymbol{S})-\mathbf{1 6 b}]$

Foam (168 mg, 89\%); HPLC $t_{\mathrm{R}} 18.57 \mathrm{~min} ;[\alpha]_{\mathrm{D}}{ }^{20}+3.5$ (c 1.1, MeOH); ${ }^{1} \mathrm{H}$ NMR (300 $\left.\mathrm{MHz}, \mathrm{CDCl}_{3}\right) \delta$ 0.77-2.23 [m, 6H, $\beta-\mathrm{H}, \gamma-\mathrm{H}$ and $\delta-\mathrm{H}$ (Lys)], 1.45 (s, 9H, Boc), 2.58-2.73 [m, $3 \mathrm{H}, 2-\mathrm{H}$ and 3-H (Pr)], 2.73 [t, 2H, J= 7 Hz, 2-H (ethyl)], 2.94-3.28 [m, 4H, 1-H (Pr) and $\varepsilon-\mathrm{H}$ (Lys)], 3.28-3.37 [m, 1H, $\alpha-\mathrm{H}$ (Lys)], 3.40 [dd, 2H, J= 7 and $13 \mathrm{~Hz}, 1-\mathrm{H}$ (ethyl)], 3.89 [dd, J= 7 and $15 \mathrm{~Hz}, \mathrm{CH}_{2}(\mathrm{Bn})$ ], 4.34 [dd, $\mathrm{J}=7$ and $15 \mathrm{~Hz}, \mathrm{CH}_{2}(\mathrm{Bn})$ ], 4.88 (bs, 1H, NH-Boc), 5.26 and $5.41(2 \mathrm{bs}, 2 \mathrm{H}, \mathrm{HNCONH}), 6.88-7.60(\mathrm{~m}, 15 \mathrm{H}$, aromatics and $N H-\mathrm{Bn}) ;{ }^{13} \mathrm{C} \mathrm{NMR}(75$ $\left.\mathrm{MHz}, \mathrm{CDCl}_{3}\right) \delta 23.6\left(\mathrm{CH}_{2}, \mathrm{C}_{\gamma}\right), 28.8\left(3 \mathrm{CH}_{3}, \mathrm{Boc}\right), 30.1\left(\mathrm{CH}_{2}, \mathrm{C}_{\delta}\right), 30.5\left(\mathrm{CH}_{2}, \mathrm{C}_{\beta}\right), 33.6\left[\mathrm{CH}_{2}\right.$, $\mathrm{C}_{2}$ (ethyl)], $36.2\left(\mathrm{CH}_{2}, \mathrm{C}_{3}\right), 40.5\left(\mathrm{CH}_{2}, \mathrm{C}_{\varepsilon}\right), 40.9\left[\mathrm{CH}_{2}, \mathrm{C}_{1}\right.$ (ethyl) $], 42.0\left(\mathrm{CH}_{2}, \mathrm{Bn}\right), 43.1\left(\mathrm{CH}_{2}\right.$, $\left.\mathrm{C}_{1}\right), 60.6\left(\mathrm{CH}, \mathrm{C}_{2}\right), 60.8\left(\mathrm{CH}, \mathrm{C}_{\alpha}\right), 79.8(\mathrm{C}, \mathrm{Boc}), 115.5-139.6(14 \mathrm{CH}$ and $4 \mathrm{C}$, aromatics), 157.1 (CO, Boc), $160.3(\mathrm{HNCONH}), 175.0(\mathrm{CONH})$; ES-MS $\mathrm{m} / \mathrm{z}[\mathrm{M}+1]^{+}$calcd for $\mathrm{C}_{36} \mathrm{H}_{48} \mathrm{FN}_{5} \mathrm{O}_{4}, 634.37$; found, 634.43 (100\%); Anal. calcd. for $\mathrm{C}_{36} \mathrm{H}_{48} \mathrm{FN}_{5} \mathrm{O}_{4}: \mathrm{C}, 68.22 ; \mathrm{H}$, 7.63; N, 11.05. Found: C, 68.36; H, 7.78; N, 10.95 .

4.5. General procedure for the $\mathrm{N}-$ Boc-deprotection of protected ureas $(\boldsymbol{R S})-\mathbf{9 b},(\boldsymbol{R})-$ and $(\boldsymbol{S})$ 8a,b, $(R)-,(S)-9 a$ and -13a, $(R)$ - and $(S)-14-16 b$. Synthesis of urea hydrochlorides $(\boldsymbol{R})-$ and $(S)-11 a,(R S)-11 b,(R)-$ and $(S)-17 \mathbf{a}, \mathbf{b},(R)-$ and $(S)-18 a,(R)-$ and $(S)-19-21 b$

The corresponding protected urea $(0.10 \mathrm{mmol})$ was dissolved in a $3 \mathrm{M}$ solution of $\mathrm{HCl}$ in EtOAc $(2 \mathrm{~mL})$ and stirred at $\mathrm{rt}$ for $2 \mathrm{~h}$. Then, the solvent was evaporated under reduced pressure, the residue was dissolved in $\mathrm{H}_{2} \mathrm{O}(3 \mathrm{~mL})$ and the solution was lyophilized.

4.5.1. $\mathrm{N}_{\alpha}$-[(2R)-4-Phenyl-1-(3-phenylureido)butan-2-yl]-ornithine benzyl amide dihydrochloride $[(\boldsymbol{R})-\mathbf{1 1 a}]$

Amorphous solid (56 mg, $100 \%) ;[\alpha]_{\mathrm{D}}{ }^{20}+6.9$ (c 0.9, MeOH); ${ }^{1} \mathrm{H}$ NMR (400 MHz, DMSO-d $\left.{ }_{6}\right) \delta 1.51-2.06[\mathrm{~m}, 6 \mathrm{H}, 3-\mathrm{H}(\mathrm{Bu}), \gamma-$ and $\beta-\mathrm{H}(\mathrm{Orn})], 2.64-2.94[\mathrm{~m}, 4 \mathrm{H}, 4-\mathrm{H}(\mathrm{Bu})$ and $\delta-\mathrm{H}(\mathrm{Orn})], 3.08$ [m, 1H, 2-H (Bu)], 3.22-3.45 [m, 2H, 1-H (Bu)], 4.09 [m, 1H, $\alpha-\mathrm{H}(\mathrm{Orn})]$, 
$4.25\left[\mathrm{dd}, 1 \mathrm{H}, \mathrm{J}=5\right.$ and $\left.15 \mathrm{~Hz}, \mathrm{CH}_{2}(\mathrm{Bn})\right], 4.40$ [dd, $1 \mathrm{H}, \mathrm{J}=4$ and $\left.15 \mathrm{~Hz}, \mathrm{CH}_{2}(\mathrm{Bn})\right], 6.74$ and 6.91 (2 bs, 2H, HNCONH), 6.97-7.47 (m, 15H, Ph), 7.95 [bs, 3H, $\left.\delta-\mathrm{NH}_{3}{ }^{+}(\mathrm{Orn})\right], 9.11$ and 9.43 [2bs, $2 \mathrm{H}, \alpha-\mathrm{NH}_{2}{ }^{+}$(Orn)], 9.33 (bs, $\left.1 \mathrm{H}, \mathrm{NH}-\mathrm{Bn}\right)$ ) ${ }^{13} \mathrm{C}$ NMR (100 MHz, DMSO-d 6 ) $\delta 22.7$ $\left(\mathrm{CH}_{2}, \mathrm{C}_{\gamma}\right), 27.2\left(\mathrm{CH}_{2}, \mathrm{C}_{\beta}\right), 28.1\left(\mathrm{CH}_{2}, \mathrm{C}_{4}\right), 29.4\left(\mathrm{CH}_{2}, \mathrm{C}_{3}\right), 31.0\left(\mathrm{CH}_{2}, \mathrm{C}_{\delta}\right), 38.1\left[\mathrm{CH}_{2},(\mathrm{Bn})\right]$, $42.6\left(\mathrm{CH}_{2}, \mathrm{C}_{1}\right), 56.6\left(\mathrm{CH}, \mathrm{C}_{2}\right), 57.8\left(\mathrm{CH}, \mathrm{C}_{\alpha}\right), 118.7-140.7(15 \mathrm{CH}$, and $3 \mathrm{C}, \mathrm{Ph}), 156.5$ (HNCONH), $166.9(\mathrm{CONH})$; ES-MS $m / z[\mathrm{M}+1]^{+}$calcd. for $\mathrm{C}_{29} \mathrm{H}_{37} \mathrm{~N}_{5} \mathrm{O}_{2}, 488.29$; found, $488.50(100 \%)$.

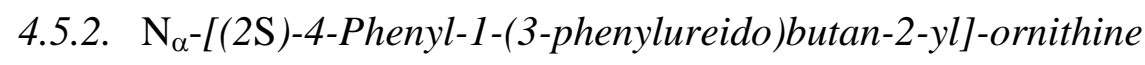
benzyl amide dihydrochloride $[(\boldsymbol{S})-\mathbf{1 1 a}]$

Amorphous solid (55 mg, $100 \%) ;[\alpha]_{\mathrm{D}}{ }^{20}+11.3\left(c\right.$ 1, MeOH); ${ }^{1} \mathrm{H}$ NMR (400 MHz, DMSO-d $\left._{6}\right) \delta 1.61-2.09[\mathrm{~m}, 6 \mathrm{H}, 3-\mathrm{H}(\mathrm{Bu}), \gamma-$ and $\beta-\mathrm{H}(\mathrm{Orn})], 2.55-2.96[\mathrm{~m}, 4 \mathrm{H}, 4-\mathrm{H}(\mathrm{Bu})$ and $\delta$-H (Orn)], 3.05 [m, 1H, 2-H (Bu)], 3.35-3.71 [m, 2H, 1-H (Bu)], 4.25 [m, 1H, $\alpha-\mathrm{H}(\mathrm{Orn})]$, $4.33\left[\mathrm{~d}, 2 \mathrm{H}, \mathrm{J}=5, \mathrm{CH}_{2}(\mathrm{Bn})\right], 6.92$ and 7.32 (2 bs, 2H, HNCONH), 7.12-7.64 (m, 15H, Ph), 8.07 [bs, 3H, $\left.\delta-\mathrm{NH}_{3}{ }^{+}(\mathrm{Orn})\right], 9.11$ and 9.43 [2bs, 2H, $\alpha-\mathrm{NH}_{2}{ }^{+}$(Orn)], 9.19 (bs, 1H, NH-Bn); ${ }^{13} \mathrm{C}$ NMR (100 MHz, DMSO-d $\left.{ }_{6}\right) \delta 22.4\left(\mathrm{CH}_{2}, \mathrm{C}_{\gamma}\right), 27.1\left(2 \mathrm{CH}_{2}, \mathrm{C}_{4}\right.$ and $\left.\mathrm{C}_{\beta}\right), 30.5\left(\mathrm{CH}_{2}, \mathrm{C}_{3}\right)$, $30.9\left(\mathrm{CH}_{2}, \mathrm{C}_{\delta}\right), 37.8\left[\mathrm{CH}_{2},(\mathrm{Bn})\right], 42.4\left(\mathrm{CH}_{2}, \mathrm{C}_{1}\right), 57.2\left(\mathrm{CH}, \mathrm{C}_{2}\right), 57.5\left(\mathrm{CH}, \mathrm{C}_{\alpha}\right), 117.7-139.9$ $(15 \mathrm{CH}$, and $3 \mathrm{C}, \mathrm{Ph}), 156.0(\mathrm{HNCONH}), 166.5(\mathrm{CONH})$; ES-MS $\mathrm{m} / \mathrm{z} \cdot[\mathrm{M}+1]^{+}$calcd. for $\mathrm{C}_{29} \mathrm{H}_{37} \mathrm{~N}_{5} \mathrm{O}_{2}$, 488.29; found, 488.50 (100\%).

4.5.3. $\mathrm{N}_{\alpha}$-[4-Phenyl-1-(3-phenylureido)butan-2-yl]-lysine benzyl amide dihydrochloride $[(R S)-11 b]$

Amorphous solid (57 mg, $29 \%)$; HPLC $t_{\mathrm{R}} 13.26[(\boldsymbol{R})-9 \mathbf{b}]$ and $13.45 \mathrm{~min}[(\boldsymbol{S})-9 \mathbf{b}] ;{ }^{1} \mathrm{H}$ NMR (400 MHz, DMSO-d d $_{6} \delta$ 1.23-1.40 [m, 2H, $\gamma-\mathrm{H}$ (Lys)], 1.43-1.62 [m, 2H, $\beta-\mathrm{H}$ (Lys)], 1.70-1,96 [m, 4H, $\delta-\mathrm{H}(\mathrm{Lys})$ and 3-H $(\mathrm{Bu})], 2.58-2.78[\mathrm{~m}, 4 \mathrm{H}, 4-\mathrm{H}(\mathrm{Bu})$ and $\varepsilon-\mathrm{H}(\mathrm{Lys})], 2.91-$ $3.06[\mathrm{~m}, 1 \mathrm{H}, 2-\mathrm{H}(\mathrm{Bu})]$, and $\alpha-\mathrm{H}(\mathrm{Lys})], 3.38-3.65[\mathrm{~m}, 2 \mathrm{H}, 1-\mathrm{H}(\mathrm{Bu})], 4.03$ and $4.16[2 \mathrm{~m}, 1 \mathrm{H}$, $\alpha-\mathrm{H}(\mathrm{Lys})], 4.26$ [dd, 0.5H, J= 7 and $\left.15 \mathrm{~Hz}, \mathrm{CH}_{2}(\mathrm{Bn})\right], 4.30$ [d, 1H, J = 5 Hz, $\left.\mathrm{CH}_{2}(\mathrm{Bn})\right], 4.35$ [dd, 0.5H, J= 7 and $\left.15 \mathrm{~Hz}, \mathrm{CH}_{2}(\mathrm{Bn})\right], 6.77$ (bs, 2H, HNCONH), 7.10-7.43 (m, $15 \mathrm{H}, \mathrm{Ph}$ ), $7.92\left[\mathrm{bs}, 3 \mathrm{H}, \delta-\mathrm{NH}_{3}{ }^{+}\right.$(Lys)], 8.93, 8.77, 9.33 and 9.46 [4bs, 2H, $\alpha-\mathrm{NH}_{2}{ }^{+}$(Lys)], 9.32 (bs, $1 \mathrm{H}$, $N H-\mathrm{Bn}) ;{ }^{13} \mathrm{C}$ NMR (100 MHz, DMSO-d $\left.{ }_{6}\right) \delta 22.9\left(\mathrm{CH}_{2}, \mathrm{C}_{\gamma}\right), 29.8\left(\mathrm{CH}_{2}, \mathrm{C}_{\delta}\right), 32.1\left(\mathrm{CH}_{2}, \mathrm{C}_{4}\right)$, $33.0\left(\mathrm{CH}_{2}, \mathrm{C}_{\beta}\right), 34.8\left(\mathrm{CH}_{2}, \mathrm{C}_{3}\right), 40.0\left(\mathrm{CH}_{2}, \mathrm{C}_{\varepsilon}\right), 42.0$ and $43.0\left(\mathrm{CH}_{2}, \mathrm{C}_{1}\right), 43.3\left[\mathrm{CH}_{2},(\mathrm{Bn})\right]$, 56.9 and $58.8\left(\mathrm{CH}, \mathrm{C}_{2}\right), 60.1\left(\mathrm{CH}, \mathrm{C}_{\alpha}\right), 119.1-141.3(15 \mathrm{CH}$, and $3 \mathrm{C}, \mathrm{Ph}), 156.2$ and 156.7 
(HNCONH), 174.9 (CONH); ES-MS m/z [M+1] $]^{+}$calcd. for $\mathrm{C}_{30} \mathrm{H}_{39} \mathrm{~N}_{5} \mathrm{O}_{2}, 502.31$; found, $502.50(100 \%)$.

4.5.4. $\mathrm{N}_{\alpha}-[(2 \mathrm{R})-3-P h e n y l-1-(3-p h e n y l u r e i d o)$ propan-2-yl]-ornithine benzyl amide dihydrochloride $[(\boldsymbol{R})-\mathbf{1 7 a}]$

Amorphous solid (54 mg, $100 \%)$; HPLC $t_{\mathrm{R}} 12.87 \mathrm{~min} ;[\alpha]_{\mathrm{D}}{ }^{20}+5.5(c 1.3, \mathrm{MeOH}) ;{ }^{1} \mathrm{H}$ NMR (400 MHz, DMSO-d $\left.{ }_{6}\right) \delta 1.56-1.79$ [m, 2H, $\gamma-\mathrm{H}($ Orn)], 1.80-2.07 [m, 2H, $\beta-\mathrm{H}$ (Orn)], 2.69-3.31 [m, 7H, 1-H, 2-H, 3-H (Pr) and $\delta-\mathrm{H}(\mathrm{Orn})], 4.25\left[\mathrm{dd}, 1 \mathrm{H}, \mathrm{J}=6\right.$ and $15 \mathrm{~Hz}, \mathrm{CH}_{2}$ (Bn)], 4.33 [m, 2H, $\alpha-\mathrm{H}(\mathrm{Orn})], 4.50$ [dd, 1H, J=6 and $\left.15 \mathrm{~Hz}, \mathrm{CH}_{2}(\mathrm{Bn})\right], 6.89$ (bs, 2H, NHCONH), 6.79-7.48 (m, 15H, aromatics), 8.08 [bs, 3H, $\left.\delta-\mathrm{NH}_{3}{ }^{+}(\mathrm{Orn})\right], 9.15$ and 9.78 [2bs, $2 \mathrm{H}, \alpha-\mathrm{NH}_{2}{ }^{+}$(Orn)], 9.65 (bs, 1H, NH-Bn); ${ }^{13} \mathrm{C}$ NMR (100 MHz, DMSO-d 6 ) $\delta 22.1\left(\mathrm{CH}_{2}, \mathrm{C}_{\gamma}\right)$, $27.1\left(\mathrm{CH}_{2}, \mathrm{C}_{\beta}\right), 28.2\left(\mathrm{CH}_{2}, \mathrm{C}_{3}\right), 33.5\left(\mathrm{CH}_{2}, \mathrm{C}_{\delta}\right), 38.0\left(\mathrm{CH}_{2}, \mathrm{Bn}\right), 42.6\left(\mathrm{CH}_{2}, \mathrm{C}_{1}\right), 56.7(\mathrm{CH}$, $\left.\mathrm{C}_{2}\right), 59.5\left(\mathrm{CH}, \mathrm{C}_{\alpha}\right), 118.1-148.0(15 \mathrm{CH}$ and $3 \mathrm{C}$, aromatics), $156.2(\mathrm{HNCONH}), 167.0$ (CONH); ES-MS $m / z[\mathrm{M}+1]^{+}$calcd for $\mathrm{C}_{28} \mathrm{H}_{35} \mathrm{~N}_{5} \mathrm{O}_{2}$, 474.28; found, 574.27 (100\%).

4.5.5. $\mathrm{N}_{\alpha}-[(2 \mathrm{~S})-3-P h e n y l-1-(3-p h e n y l u r e i d o) p r o p a n-2-y l]$-ornithine benzyl amide dihydrochloride $[(\boldsymbol{S})-\mathbf{1 7 a}]$

Amorphous solid (55 mg, $100 \%)$; HPLC $t_{\mathrm{R}} 12.89 \mathrm{~min} ;[\alpha]_{\mathrm{D}}{ }^{20}+26.6(c 0.9, \mathrm{MeOH}) ;{ }^{1} \mathrm{H}$ NMR (400 MHz, DMSO-d $\left.{ }_{6}\right) \delta$ 1.67-1.78 [m, 2H, $\left.\gamma-\mathrm{H}(\mathrm{Orn})\right], 1.82-2.06$ [m, 2H, $\left.\beta-\mathrm{H}(\mathrm{Orn})\right]$, 2.74-2.96 [m, 3H, 3-H (Pr) and $\delta-\mathrm{H}(\mathrm{Orn})], 2.99-3.18$ [m, 1H, 3-H (Pr)], $3.11[\mathrm{~m}, 1 \mathrm{H}, 1-\mathrm{H}$ (Pr)], 3.23 [m, 1H, 2-H (Pr)], 3.53 [m, 1H, 1-H (Pr)], 4.27-4.44 [m, 1H, $\alpha-\mathrm{H}($ Orn)], 4.35 [d, $\left.2 \mathrm{H}, \mathrm{J}=6.5 \mathrm{~Hz}, \mathrm{CH}_{2}(\mathrm{Bn})\right], 6.86-7.45$ (m, 15H, aromatics), 6.94 and 7.10 (2bs, 2H, NHCONH), 8.12 [bs, $3 \mathrm{H}, \delta-\mathrm{NH}_{3}{ }^{+}$(Orn)], 9.41 and 9.50 [2bs, $2 \mathrm{H}, \alpha-\mathrm{NH}_{2}{ }^{+}$(Orn)], 9.64 (bs, $\left.1 \mathrm{H}, \mathrm{NH}-\mathrm{Bn}\right)$; ${ }^{13} \mathrm{C}$ NMR $\left(100 \mathrm{MHz}, \mathrm{DMSO}-\mathrm{d}_{6}\right) \delta 22.7\left(\mathrm{CH}_{2}, \mathrm{C}_{\gamma}\right), 27.3\left(\mathrm{CH}_{2}, \mathrm{C}_{\beta}\right), 34.9\left(\mathrm{CH}_{2}, \mathrm{C}_{3}\right), 37.7\left(\mathrm{CH}_{2}\right.$, $\left.\mathrm{C}_{\delta}\right), 38.8\left(\mathrm{CH}_{2}, \mathrm{Bn}\right), 42.7\left(\mathrm{CH}_{2}, \mathrm{C}_{1}\right), 57.7\left(\mathrm{CH}, \mathrm{C}_{2}\right), 59.0\left(\mathrm{CH}, \mathrm{C}_{\alpha}\right), 117.8-140.2(15 \mathrm{CH}$ and 3 C, aromatics), $156.2(\mathrm{HNCONH}), 167.2(\mathrm{CONH})$; ES-MS $m / z[\mathrm{M}+1]^{+} \mathrm{ES}-\mathrm{MS} \mathrm{m} / \mathrm{z}[\mathrm{M}+1]^{+}$ calcd for $\mathrm{C}_{28} \mathrm{H}_{35} \mathrm{~N}_{5} \mathrm{O}_{2}$, 474.28; found, 574.52 (100\%).

4.5.6. $\mathrm{N}_{\alpha}$-[(2R)-3-Phenyl-1-(3-phenylureido)propan-2-yl]-lysine benzyl amide dihydrochloride $[(\boldsymbol{R})-\mathbf{1 7 b}]$

Amorphous solid (56 mg, 100\%); HPLC $t_{\mathrm{R}} 12.78 \mathrm{~min} ;[\alpha]_{\mathrm{D}}{ }^{20}+16.5(c 1.6, \mathrm{MeOH}) ;{ }^{1} \mathrm{H}$ NMR (400 MHz, DMSO-d ${ }_{6}$ ) $\delta$ 1.25-1.48 [m, 2H, $\gamma-\mathrm{H}$ (Lys)], 1.50-1.70 [m, 2H, $\delta$-H (Lys)], 1.72-2.08 [m, 2H, $\beta-\mathrm{H}$ (Lys)], 2.72 [m, 2H, $\varepsilon-\mathrm{H}$ (Lys)], 2.77-2.92 [m, 1H, 3-H (Pr)], 3.08-3.34 
[m, 3H, 1-H and 3-H (Pr)], 3.24 [m, 1H, 2-H (Pr)], 4.20 [m, 1H, $\alpha-\mathrm{H}(\mathrm{Lys})], 4.28$ [dd, 1H, J= 6 and $\left.15 \mathrm{~Hz}, \mathrm{CH}_{2}(\mathrm{Bn})\right], 4.45$ [dd, , 1H, J= 5 and $\left.15 \mathrm{~Hz}, \mathrm{CH}_{2}(\mathrm{Bn})\right], 6.89$ (bs, 2H, HNCONH), 7.09-7.49 (m, 15H, aromatics), 8.01 [bs, 3H, $\left.\delta-\mathrm{NH}_{3}{ }^{+}(\mathrm{Lys})\right], 9.01$ and 9.77 [2bs, $2 \mathrm{H}, \alpha-\mathrm{NH}_{2}{ }^{+}$ (Lys)], 9.47 (bs, 1H, NH-Bn); ${ }^{13} \mathrm{C}$ NMR (100 MHz, DMSO-d $) \delta 22.0\left(\mathrm{CH}_{2}, \mathrm{C}_{\gamma}\right), 27.1\left(\mathrm{CH}_{2}\right.$, $\left.\mathrm{C}_{\delta}\right), 30.2\left(\mathrm{CH}_{2}, \mathrm{C}_{\beta}\right), 34.3\left(\mathrm{CH}_{2}, \mathrm{C}_{3}\right), 38.9\left(\mathrm{CH}_{2}, \mathrm{C}_{\varepsilon}\right), 38.9\left(\mathrm{CH}_{2}, \mathrm{Bn}\right), 43.3\left(\mathrm{CH}_{2}, \mathrm{C}_{1}\right), 57.9$ $\left(\mathrm{CH}, \mathrm{C}_{2}\right), 60.3\left(\mathrm{CH}, \mathrm{C}_{\alpha}\right), 118.9-140.7$ (15CH and 3C, aromatics), 157.3 (HNCONH), 167.8 (CONH); ES-MS $m / z[\mathrm{M}+1]^{+}$calcd for $\mathrm{C}_{29} \mathrm{H}_{37} \mathrm{~N}_{5} \mathrm{O}_{2}, 488.29$; found, 488.50 (100\%).

4.5.7. $\mathrm{N}_{\alpha}$-[(2S)-3-Phenyl-1-(3-phenylureido)propan-2-yl]-lysine benzyl amide dihydrochloride $[(\boldsymbol{S})-\mathbf{1 7 b}]$

Amorphous solid (57 mg, $100 \%$ ); HPLC $t_{\mathrm{R}} 13.26 \mathrm{~min} ;[\alpha]_{\mathrm{D}}{ }^{20}+19.3(c 1.5, \mathrm{MeOH}) ;{ }^{1} \mathrm{H}$ NMR (400 MHz, DMSO-d $\left.{ }_{6}\right) \delta$ 1.28-1.49 [m, 2H, $\gamma-\mathrm{H}$ (Lys)], 1.51-1.69 [m, 2H, $\delta$-H (Lys)], 1.74-2.01 [m, 2H, $\beta-\mathrm{H}$ (Lys)], 2.69 [m, 2H, $\varepsilon-\mathrm{H}$ (Lys)], 2.78-2.94 [m, 1H, 3-H (Pr)], 2.99-3.18 [m, 2H, 1-H and 3-H (Pr)], 3.23 [m, 1H, 2-H (Pr)], 3.51-3.60 [m, 1H, 1-H (Pr)], 4.23-4.48 [m, $1 \mathrm{H}, \alpha-\mathrm{H}(\mathrm{Lys})], 4.33\left[\mathrm{~d}, 2 \mathrm{H}, \mathrm{J}=5 \mathrm{~Hz}, \mathrm{CH}_{2}(\mathrm{Bn})\right], 6.80-7.48\left[\mathrm{~m}, 18 \mathrm{H}\right.$, aromatics and $\delta-\mathrm{NH}_{3}{ }^{+}$ (Lys)], 6.89 and 7.15 (2bs, 2H, NHCONH), 9.09 and 9.51 [2bs, 2H, $\alpha-\mathrm{NH}_{2}{ }^{+}$(Lys)], 9.51 (bs, $1 \mathrm{H}, \mathrm{NH}-\mathrm{Bn}) ;{ }^{13} \mathrm{C}$ NMR $\left(100 \mathrm{MHz}, \mathrm{DMSO}-\mathrm{d}_{6}\right) \delta 22.1\left(\mathrm{CH}_{2}, \mathrm{C}_{\gamma}\right), 27.0\left(\mathrm{CH}_{2}, \mathrm{C}_{\delta}\right), 30.4\left(\mathrm{CH}_{2}\right.$, $\left.\mathrm{C}_{\beta}\right), 35.7\left(\mathrm{CH}_{2}, \mathrm{C}_{3}\right), 38.5\left(\mathrm{CH}_{2}, \mathrm{C}_{\varepsilon}\right), 38.9\left(\mathrm{CH}_{2}, \mathrm{Bn}\right), 43.3\left(\mathrm{CH}_{2}, \mathrm{C}_{1}\right), 58.9\left(\mathrm{CH}, \mathrm{C}_{2}\right), 59.8(\mathrm{CH}$, $\left.\mathrm{C}_{\alpha}\right), 118.5-140.9$ (15CH and 3C, aromatics), $157.0(\mathrm{HNCONH}), 168.0(\mathrm{CONH})$; ES-MS $\mathrm{m} / \mathrm{z}$ $[\mathrm{M}+1]^{+}$calcd for $\mathrm{C}_{29} \mathrm{H}_{37} \mathrm{~N}_{5} \mathrm{O}_{2}, 488.29$; found, 488.50 (100\%).

4.5.8. $\mathrm{N}_{\alpha}$-[(2R)-1-(3-Benzylureido)-3-phenylpropan-2-yl]-ornithine benzyl amide dihydrochloride $[(\boldsymbol{R}) \mathbf{- 1 8 a}]$

Amorphous solid (56 mg, $100 \%)$; HPLC $t_{\mathrm{R}} 12.87 \mathrm{~min} ;[\alpha]_{\mathrm{D}}{ }^{20}+5.2(c \mathrm{1}, \mathrm{MeOH}) ;{ }^{1} \mathrm{H}$ NMR (500 MHz, DMSO-d 6 ) $\delta$ 1.56-1.82 [m, 2H, $\gamma$-H (Orn)], 1.90-2.10 [m, 2H, $\beta-\mathrm{H}$ (Orn)], 2.74$3.02[\mathrm{~m}, 4 \mathrm{H}, 3-\mathrm{H}(\mathrm{Pr})$ and $\delta-\mathrm{H}(\mathrm{Orn})], 3.23$ [m, 1H, 2-H (Pr)], 3.09-3.22 [m, 2H, 1-H (Pr)], 4.30 [m, 2H, $\mathrm{CH}_{2}$ (Bn-ureido)], 4.32-4.38 [m,1H, $\left.\alpha-\mathrm{H}(\mathrm{Orn})\right], 4.35$ [dd, 1H, J=6.5 and $15 \mathrm{~Hz}$, $\mathrm{CH}_{2}(\mathrm{Bn})$ ], 4.60 [ dd, $1 \mathrm{H}, \mathrm{J}=5$ and $\left.15 \mathrm{~Hz}, \mathrm{CH}_{2}(\mathrm{Bn})\right], 6.72$ and 7.00 (2bs, 2H, NHCONH), 7.21-7.65 (m, 15H, aromatics), $8.08\left[\mathrm{bs}, 3 \mathrm{H}, \delta-\mathrm{NH}_{3}{ }^{+}(\mathrm{Orn})\right], 9.56$ and $9.81\left[2 \mathrm{bs}, 2 \mathrm{H}, \alpha-\mathrm{NH}_{2}{ }^{+}\right.$ (Orn)], 9.56 (bs, 1H, NH-Bn); ${ }^{13} \mathrm{C}$ NMR (100 MHz, DMSO-d $) \delta 22.7\left(\mathrm{CH}_{2}, \mathrm{C}_{\gamma}\right), 27.2\left(\mathrm{CH}_{2}\right.$, $\left.\mathrm{C}_{\beta}\right), 28.9\left(\mathrm{CH}_{2}, \mathrm{C}_{3}\right), 33.5\left(\mathrm{CH}_{2}, \mathrm{C}_{\delta}\right), 38.1\left(\mathrm{CH}_{2}, \mathrm{Bn}\right), 42.6\left[\mathrm{CH}_{2},(\mathrm{Bn}\right.$-ureido) $], 43.1\left(\mathrm{CH}_{2}, \mathrm{C}_{1}\right)$, $56.7\left(\mathrm{CH}, \mathrm{C}_{2}\right), 60.4\left(\mathrm{CH}, \mathrm{C}_{\alpha}\right), 126.9-140.2(15 \mathrm{CH}$ and $3 \mathrm{C}$, aromatics), 159.7 (HNCONH), $166.9(\mathrm{CONH})$; ES-MS $m / z$ [M+1] ${ }^{+}$calcd for $\mathrm{C}_{29} \mathrm{H}_{37} \mathrm{~N}_{5} \mathrm{O}_{2}, 488.29$; found, 488.52 (100\%). 
4.5.9. $\mathrm{N}_{\alpha}$-[(2S)-1-(3-Benzylureido)-3-phenylpropan-2-yl]-ornithine

amide dihydrochloride $[(\boldsymbol{S})-\mathbf{1 8 a}]$

Amorphous solid (55 mg, $100 \%)$; HPLC $t_{\mathrm{R}} 12.95 \mathrm{~min} ;[\alpha]_{\mathrm{D}}{ }^{20}+2.1(c 1.5, \mathrm{MeOH}) ;{ }^{1} \mathrm{H}$ NMR (500 MHz, DMSO-d $\left.{ }_{6}\right) \delta 1.58-1.74[\mathrm{~m}, 2 \mathrm{H}, \gamma-\mathrm{H}(\mathrm{Orn})], 1.80-1.99$ [m, 2H, $\beta-\mathrm{H}$ (Orn)], 2.74-3.10 [m, 5H, 1-H, 3-H (Pr) and $\delta-\mathrm{H}(\mathrm{Orn})], 3.18$ [m, 1H, 2-H (Pr)], 3.38-3.46 [m, 1H, 1$\mathrm{H}(\mathrm{Pr})], 4.20$ [dd, $1 \mathrm{H}, \mathrm{J}=6$ and $15 \mathrm{~Hz}, \mathrm{CH}_{2}$ (Bn-ureido)], 4.28 [d, 2H, J= $\left.6 \mathrm{CH}_{2}(\mathrm{Bn})\right], 4.32$ [m,1H, $\alpha-\mathrm{H}(\mathrm{Orn})], 4.41$ [dd, $1 \mathrm{H}, \mathrm{J}=6$ and $15 \mathrm{~Hz}, \mathrm{CH}_{2}$ (Bn-ureido)], 4.60 [ dd, 1H, J=5 and $\left.15 \mathrm{~Hz}, \mathrm{CH}_{2}(\mathrm{Bn})\right], 6.94$ and 7.11 (2bs, 2H, $\mathrm{NHCONH}$ ), 7.18-7.30 (m, 15H, aromatics), 8.09 $\left[\mathrm{bs}, 3 \mathrm{H}, \delta-\mathrm{NH}_{3}{ }^{+}(\mathrm{Orn})\right], 9.19$ and $9.86\left[2 \mathrm{bs}, 2 \mathrm{H}, \alpha-\mathrm{NH}_{2}{ }^{+}(\mathrm{Orn})\right], 9.54$ (bs, $\left.1 \mathrm{H}, \mathrm{NH}-\mathrm{Bn}\right) ;{ }^{13} \mathrm{C}$ NMR (100 MHz, DMSO-d $\left.{ }_{6}\right) \delta 23.3\left(\mathrm{CH}_{2}, \mathrm{C}_{\gamma}\right), 27.9\left(\mathrm{CH}_{2}, \mathrm{C}_{\beta}\right), 28.8\left(\mathrm{CH}_{2}, \mathrm{C}_{3}\right), 35.4\left(\mathrm{CH}_{2}, \mathrm{C}_{\delta}\right)$, $38.6\left(\mathrm{CH}_{2}, \mathrm{Bn}\right), 43.4\left[\mathrm{CH}_{2}\right.$, (Bn-ureido)], $43.7\left(\mathrm{CH}_{2}, \mathrm{C}_{1}\right), 58.5\left(\mathrm{CH}, \mathrm{C}_{2}\right), 60.6\left(\mathrm{CH}, \mathrm{C}_{\alpha}\right), 127.4-$ $140.9\left(15 \mathrm{CH}\right.$ and $3 \mathrm{C}$, aromatics), $160.5(\mathrm{HNCONH}), 167.9(\mathrm{CONH}) ; \mathrm{ES}-\mathrm{MS} \mathrm{m} / \mathrm{z}[\mathrm{M}+1]^{+}$ calcd for $\mathrm{C}_{29} \mathrm{H}_{37} \mathrm{~N}_{5} \mathrm{O}_{2}, 488.29$; found, 488.38 (100\%).

4.5.10. $\mathrm{N}_{\alpha}$-[(2R)-1-(3-(2,4-Dichlorophenyl)ureido)-3-phenylpropan-2-yl]-lysine benzyl amide dihydrochloride $[(\boldsymbol{R})-\mathbf{1 9 b}]$

Amorphous solid (62 mg, $100 \%)$; HPLC $t_{\mathrm{R}} 14.85 \mathrm{~min} ;[\alpha]_{\mathrm{D}}{ }^{20}+9.8(c 0.4, \mathrm{MeOH}) ;{ }^{1} \mathrm{H}$ NMR (500 MHz, DMSO-d $\left.{ }_{6}\right) \delta$ 1.37-1.49 [m, 2H, $\gamma-\mathrm{H}$ (Lys)], 1.53-1.71 [m, 2H, $\delta-\mathrm{H}$ (Lys)], 1.82-2.05 [m, 2H, $\beta-\mathrm{H}$ (Lys)], 2.80 [m, 2H, $\varepsilon-\mathrm{H}$ (Lys)], 2.91 [m, 1H, 3-H (Pr)], 3.23 [m, 1H, 2-H (Pr)], 3.27-3.40 [m, 3H, 1-H and 3-H (Pr)], 3.26 [m, 1H, $\alpha-\mathrm{H}(\mathrm{Lys})], 3.26$ [dd, 1H, J= 6 and $\left.15 \mathrm{~Hz}, \mathrm{CH}_{2}(\mathrm{Bn})\right], 4.52\left[\mathrm{dd},, 1 \mathrm{H}, \mathrm{J}=5\right.$ and $\left.15 \mathrm{~Hz}, \mathrm{CH}_{2}(\mathrm{Bn})\right], 7.18-7.44(\mathrm{~m}, 13 \mathrm{H}$, aromatics), 7.59 (bs, 2H, HNCONH), 7.94 [bs, 3H, $\delta-\mathrm{NH}_{3}{ }^{+}$(Lys)], 8.99 and $9.67[2 \mathrm{bs}, 2 \mathrm{H}, \alpha-$ $\mathrm{NH}_{2}{ }^{+}$(Lys)], 9.43 (bs, 1H, NH-Bn); ${ }^{13} \mathrm{C}$ NMR (125 MHz, DMSO-d 6 ) $\delta 21.3\left(\mathrm{CH}_{2}, \mathrm{C}_{\gamma}\right), 26.5$ $\left(\mathrm{CH}_{2}, \mathrm{C}_{\delta}\right), 29.4\left(\mathrm{CH}_{2}, \mathrm{C}_{\beta}\right), 32.0\left(\mathrm{CH}_{2}, \mathrm{C}_{3}\right), 37.5\left(\mathrm{CH}_{2}, \mathrm{C}_{\varepsilon}\right), 38.3\left(\mathrm{CH}_{2}, \mathrm{Bn}\right), 42.6\left(\mathrm{CH}_{2}, \mathrm{C}_{1}\right)$, $57.2\left(\mathrm{CH}, \mathrm{C}_{2}\right), 58.8\left(\mathrm{CH}, \mathrm{C}_{\alpha}\right), 116.9-140.2(13 \mathrm{CH}$ and $5 \mathrm{C}$, aromatics), $155.9(\mathrm{HNCONH})$, 167.1 (CONH); ES-MS $m / z[\mathrm{M}+1]^{+}$calcd for $\mathrm{C}_{29} \mathrm{H}_{35} \mathrm{Cl}_{2} \mathrm{~N}_{5} \mathrm{O}_{2}, 556.22$; found, 556.63 (100\%), $558.52(64 \%)[\mathrm{M}+3]^{+}, 560.42(10 \%)[\mathrm{M}+5]^{+}$.

4.5.11. $\mathrm{N}_{\alpha}$-[(2S)-1-(3-(2,4-Dichlorophenyl)ureido)-3-phenylpropan-2-yl]-lysine benzyl amide dihydrochloride $[(\boldsymbol{S}) \mathbf{- 1 9 b}]$

Amorphous solid (63 mg, $100 \%$ ); HPLC $t_{\mathrm{R}} 14.09 \mathrm{~min} ;[\alpha]_{\mathrm{D}}{ }^{20}+12.3(c 1.1, \mathrm{MeOH}) ;{ }^{1} \mathrm{H}$ NMR (400 MHz, DMSO-d $\left.{ }_{6}\right) \delta$ 1.17-1.42 [m, 2H, $\gamma-\mathrm{H}$ (Lys)], 1.46-1.681 [m, 2H, $\delta$-H (Lys)], 
1.68-1.98 [m, 2H, $\beta-\mathrm{H}$ (Lys)], 2.61-2.95 [m, 2H, 3-H (Pr)], 2.71 [m, 2H, $\varepsilon-\mathrm{H}$ (Lys)], 2.963.19 [m, 2H, 1-H (Pr)], 3.23 [m, 1H, 2-H (Pr)], 4.3 [m, 1H, $\alpha-\mathrm{H}(\mathrm{Lys})], 4.36$ [d, 2H, J= 5 Hz, $\mathrm{CH}_{2}(\mathrm{Bn})$ ], 7.11-7.39 (m, 15H, aromatics and $\mathrm{HNCONH}$ ), 7.92 [bs, 3H, $\delta-\mathrm{NH}_{3}{ }^{+}$(Lys)], 9.16 [bs, 2H, $\alpha-\mathrm{NH}_{2}{ }^{+}$(Lys)], 9.41 (bs, 1H, NH-Bn); ${ }^{13} \mathrm{C}$ NMR (100 MHz, DMSO-d $\left.{ }_{6}\right) \delta 21.9\left(\mathrm{CH}_{2}\right.$, $\left.\mathrm{C}_{\gamma}\right), 26.9\left(\mathrm{CH}_{2}, \mathrm{C}_{\delta}\right), 29.5\left(\mathrm{CH}_{2}, \mathrm{C}_{\beta}\right), 35.0\left(\mathrm{CH}_{2}, \mathrm{C}_{3}\right), 38.3\left(\mathrm{CH}_{2}, \mathrm{Bn}\right), 38.8\left(\mathrm{CH}_{2}, \mathrm{C}_{\varepsilon}\right), 43.1$ $\left(\mathrm{CH}_{2}, \mathrm{C}_{1}\right), 58.5\left(\mathrm{CH}, \mathrm{C}_{2}\right), 59.0\left(\mathrm{CH}, \mathrm{C}_{\alpha}\right), 116.5-141.5$ (13CH and $5 \mathrm{C}$, aromatics), 156.2 (HNCONH), $167.9(\mathrm{CONH})$; ES-MS $\mathrm{m} / z[\mathrm{M}+1]^{+}$calcd for $\mathrm{C}_{29} \mathrm{H}_{35} \mathrm{Cl}_{2} \mathrm{~N}_{5} \mathrm{O}_{2}, 556.22$; found, $556.63(100 \%), 558.52(64 \%)[\mathrm{M}+3]^{+}, 560.42(10 \%)[\mathrm{M}+5]^{+}$.

4.5.12. $\mathrm{N}_{\alpha}$-[(2R)-1-(3-(4-Methoxyphenethyl)ureido)-3-phenylpropan-2-yl]-lysine benzyl amide dihydrochloride $[(\boldsymbol{R})-\mathbf{2 0 b}]$

Amorphous solid (61 mg, $100 \%)$; HPLC $t_{\mathrm{R}} 13.46 \mathrm{~min} ;[\alpha]_{\mathrm{D}}{ }^{20}+15.6(c 0.8, \mathrm{MeOH}) ;{ }^{1} \mathrm{H}$ NMR (500 MHz, DMSO-d 6 ) $\delta$ 1.27-1.39 [m, 2H, $\gamma-\mathrm{H}$ (Lys)], 1.39-1.64 [m, 2H, $\delta$-H (Lys)], 1.74-1.95 [m, 2H, $\beta-\mathrm{H}$ (Lys)], 2.68-2.79 [m, 1H, 3-H (Pr)], 2.63 [t, 2H, J= 7 Hz, 2-H (ethyl)], $2.71[\mathrm{~m}, 2 \mathrm{H}, \varepsilon-\mathrm{H}$ (Lys)], 3.03-3.25 [m, 6H, 1-H, 2-H, 3-H (Pr) and 1-H (ethyl)], 3.69 (s, 3H, OMe), 4.09 [m, 1H, $\alpha-\mathrm{H}$ (Lys)], 4.28 [dd, 1H, J= 6 and $\left.15 \mathrm{~Hz}, \mathrm{CH}_{2}(\mathrm{Bn})\right], 4.47$ [dd, 1H, J= 5 and $\left.15 \mathrm{~Hz}, \mathrm{CH}_{2}(\mathrm{Bn})\right], 6.37$ and 6.50 (2bs, 2H, $\left.\mathrm{HNCONH}\right), 6.69-7.38$ (m, 14H, aromatics), 7.80 [bs, 3H, $\delta-\mathrm{NH}_{3}{ }^{+}$(Lys)], 9.25 [bs, $2 \mathrm{H}, \alpha-\mathrm{NH}_{2}{ }^{+}$(Lys)], 9.45 (bs, $1 \mathrm{H}, \mathrm{NH}-\mathrm{Bn}$ ); ${ }^{13} \mathrm{C} \mathrm{NMR}$ $\left(125 \mathrm{MHz}, \mathrm{DMSO}-\mathrm{d}_{6}\right) \delta 21.6\left(\mathrm{CH}_{2}, \mathrm{C}_{\gamma}\right), 27.2\left(\mathrm{CH}_{2}, \mathrm{C}_{\delta}\right), 30.5\left(\mathrm{CH}_{2}, \mathrm{C}_{\beta}\right), 34.3\left(\mathrm{CH}_{2}, \mathrm{C}_{3}\right), 35.4$ $\left[\mathrm{CH}_{2}, \mathrm{C}_{2}\right.$ (ethyl)], $38.0\left[\mathrm{CH}_{2}, \mathrm{C}_{\varepsilon}\right.$ and $\mathrm{C}_{1}$ (ethyl)], $38.0\left(\mathrm{CH}_{2}, \mathrm{C}_{\varepsilon}\right), 38.8\left(\mathrm{CH}_{2}, \mathrm{Bn}\right), 41.9\left(\mathrm{CH}_{2}\right.$, $\left.\mathrm{C}_{1}\right), 55.4\left(\mathrm{CH}, \mathrm{C}_{2}\right), 55.4\left(\mathrm{CH}_{3}, \mathrm{OMe}\right), 56.8\left(\mathrm{CH}, \mathrm{C}_{\alpha}\right), 114.0-158.0(14 \mathrm{CH}$ and $4 \mathrm{C}$, aromatics), $161.5(\mathrm{HNCONH}), 167.9(\mathrm{CONH})$; ES-MS $\mathrm{m} / z[\mathrm{M}+1]^{+}$calcd for $\mathrm{C}_{32} \mathrm{H}_{43} \mathrm{~N}_{5} \mathrm{O}_{3}, 546.34$; found, $546.62(100 \%)$.

4.5.13. $\mathrm{N}_{\alpha}$-[(2S)-1-(3-(4-Methoxyphenethyl)ureido)-3-phenylpropan-2-yl]-lysine

benzyl amide dihydrochloride $[(\boldsymbol{S})-\mathbf{2 0 b}]$

Amorphous solid (62 mg, $100 \%)$; HPLC $t_{\mathrm{R}} 13.52 \mathrm{~min} ;[\alpha]_{\mathrm{D}}{ }^{20}+19.7(c 0.7, \mathrm{MeOH}) ;{ }^{1} \mathrm{H}$ NMR (500 MHz, DMSO-d 6 ) $\delta 1.35$ [m, 2H, $\gamma$-H (Lys)], 1.594 [m, 2H, $\delta$-H (Lys)], 1.83 [m, 2H, $\beta-\mathrm{H}$ (Lys)], 2.62 [m, 2H, $\varepsilon-\mathrm{H}$ (Lys)], 2.71 [m, 2H, 2-H (ethyl)], 2.96 [m, 1H, 3-H (Pr)], $3.03[\mathrm{~m}, 3 \mathrm{H}, 1-\mathrm{H}$ and 3-H (Pr)], 3.15 [d, 2H, J= $7 \mathrm{~Hz}, 1-\mathrm{H}$ (ethyl)], 3.20 [m, 1H, 2-H (Pr)], 3.69 (s, 3H, OMe), 4.21 [m, 1H, $\alpha-\mathrm{H}$ (Lys)], 4.36 [m, 2H, $\left.\mathrm{CH}_{2}(\mathrm{Bn})\right], 6.57$ and $6.76(2 \mathrm{bs}, 2 \mathrm{H}$, HNCONH), 7.14-7.3 (m, 14H, aromatics), 8.03 [bs, 3H, $\delta-\mathrm{NH}_{3}{ }^{+}$(Lys)], 9.00 and 9.92 [2bs, $2 \mathrm{H}, \alpha-\mathrm{NH}_{2}{ }^{+}$(Lys)], 9.45 (bs, 1H, NH-Bn); ${ }^{13} \mathrm{C}$ NMR (125 MHz, DMSO-d 6 ) $\delta 21.3\left(\mathrm{CH}_{2}, \mathrm{C}_{\gamma}\right)$, 
$26.3\left(\mathrm{CH}_{2}, \mathrm{C}_{\delta}\right), 29.6\left(\mathrm{CH}_{2}, \mathrm{C}_{\beta}\right), 34.7\left[\mathrm{CH}_{2}, \mathrm{C}_{2}\right.$ (ethyl)], $34.9\left(\mathrm{CH}_{2}, \mathrm{C}_{3}\right), 38.1\left[\mathrm{CH}_{2}, \mathrm{C}_{1}\right.$ (ethyl)], $38.7\left(\mathrm{CH}_{2}, \mathrm{C}_{\varepsilon}\right), 40.1\left(\mathrm{CH}_{2}, \mathrm{Bn}\right), 42.6\left(\mathrm{CH}_{2}, \mathrm{C}_{1}\right), 54.9\left(\mathrm{CH}_{3}, \mathrm{OMe}\right), 58.4\left(\mathrm{CH}, \mathrm{C}_{2}\right), 60.0(\mathrm{CH}$, $\left.\mathrm{C}_{\alpha}\right), 113.8-157.6(14 \mathrm{CH}$ and $4 \mathrm{C}$, aromatics), $159.8(\mathrm{HNCONH}), 167.4(\mathrm{CONH}) ; \mathrm{ES}-\mathrm{MS} \mathrm{m} / \mathrm{z}$ $[\mathrm{M}+1]^{+}$calcd for $\mathrm{C}_{32} \mathrm{H}_{43} \mathrm{~N}_{5} \mathrm{O}_{3}, 546.34$; found, 546.62(100\%).

4.5.14. $\mathrm{N}_{\alpha}-[(2 \mathrm{R})-1-(3-(4-F l u o r o p h e n e t h y l)$ ureido)-3-phenyl)propan-2-yl]-lysine benzyl amide dihydrochloride $[(\boldsymbol{R})-\mathbf{2 1 b}]$

Amorphous solid (60 mg, $100 \%)$; HPLC $t_{\mathrm{R}} 13.65 \mathrm{~min} ;[\alpha]_{\mathrm{D}}{ }^{20}+15.2(c 0.8, \mathrm{MeOH}) ;{ }^{1} \mathrm{H}$ NMR (500 MHz, DMSO-d d $_{6} \delta$ 1.30-1.41 [m, 2H, $\gamma-\mathrm{H}$ (Lys)], 1.50-1.67 [m, 2H, $\delta$-H (Lys)], 1.75-1.98 [m, 2H, $\beta-\mathrm{H}$ (Lys)], 2.68 [t, 2H, J= 7 Hz, 2-H (ethyl)], 2.71 [m, 2H, $\varepsilon-\mathrm{H}$ (Lys)], $2.78[\mathrm{~m}, 1 \mathrm{H}, 3-\mathrm{H}(\mathrm{Pr})], 3.04[\mathrm{~m}, 1 \mathrm{H}, 2-\mathrm{H}(\mathrm{Pr})], 3.08-3.26[\mathrm{~m}, 5 \mathrm{H}, 1-\mathrm{H}, 3-\mathrm{H}(\mathrm{Pr})$, and 1-H (ethyl)], 4.21 [m, 1H, $\alpha-\mathrm{H}$ (Lys)], 4.28 [dd, 1H, J= 6.5 and $15 \mathrm{~Hz}, \mathrm{CH}_{2}(\mathrm{Bn})$ ], 4.46 [dd, 1H, J= 5 and $\left.15 \mathrm{~Hz}, \mathrm{CH}_{2}(\mathrm{Bn})\right], 6.43$ and 6.59 (2bs, 2H, $\left.\mathrm{HNCONH}\right), 7.02-7.36$ (m, 14H, aromatics), $7.95\left[\mathrm{bs}, 3 \mathrm{H}, \delta-\mathrm{NH}_{3}{ }^{+}\right.$(Lys)], 9.42 and 9.84 [2bs, 2H, $\alpha-\mathrm{NH}_{2}{ }^{+}$(Lys)], 9.48 (bs, 1H, NH-Bn); ${ }^{13} \mathrm{C}$ NMR $\left(75 \mathrm{MHz}, \mathrm{DMSO}-\mathrm{d}_{6}\right) \delta 21.6\left(\mathrm{CH}_{2}, \mathrm{C}_{\gamma}\right), 26.8\left(\mathrm{CH}_{2}, \mathrm{C}_{\delta}\right), 30.0\left(\mathrm{CH}_{2}, \mathrm{C}_{\beta}\right), 33.9\left(\mathrm{CH}_{2}\right.$, $\left.\mathrm{C}_{3}\right), 35.3\left[\mathrm{CH}_{2}, \mathrm{C}_{2}\right.$ (ethyl)], $38.2\left[\mathrm{CH}_{2}, \mathrm{C}_{1}\right.$ (ethyl)], $38.7\left(\mathrm{CH}_{2}, \mathrm{C}_{\varepsilon}\right), 41.7\left(\mathrm{CH}_{2}, \mathrm{Bn}\right), 42.9\left(\mathrm{CH}_{2}\right.$, $\left.\mathrm{C}_{1}\right), 57.6\left(\mathrm{CH}, \mathrm{C}_{2}\right), 61.2\left(\mathrm{CH}, \mathrm{C}_{\alpha}\right), 115.2-160.1$ (14CH and $4 \mathrm{C}$, aromatics), $162.2(\mathrm{HNCONH})$, $167.5(\mathrm{CONH})$; ES-MS $m / z[\mathrm{M}+1]^{+}$calcd for $\mathrm{C}_{31} \mathrm{H}_{40} \mathrm{FN}_{5} \mathrm{O}_{2}, 534.32$; found, 534.65 (100\%).

4.5.15. $\mathrm{N}_{\alpha}$-[(2S)-1-(3-(4-Fluorophenethyl)ureido)-3-phenyl)propan-2-yl]-lysine benzyl amide dihydrochloride $[(\boldsymbol{S})-\mathbf{2 1} \mathbf{b}]$

Amorphous solid (59 mg, $100 \%)$; HPLC $t_{\mathrm{R}} 13.69 \mathrm{~min} ;[\alpha]_{\mathrm{D}}{ }^{20}+21.5(c 1.3, \mathrm{MeOH}) ;{ }^{1} \mathrm{H}$ NMR (500 MHz, DMSO-d $\left.{ }_{6}\right) \delta 1.36$ [m, 2H, $\gamma-\mathrm{H}$ (Lys)], 1.59 [m, 2H, $\delta$-H (Lys)], 1.84 [m, 2H, $\beta-\mathrm{H}$ (Lys)], 2.68 [t, 2H, J= 7 Hz, 2-H (ethyl)], 2.72 [m, 2H, $\varepsilon-\mathrm{H}$ (Lys)], 2.78 [m, 1H, 3-H (Pr)], 2.97 [m, 1H, 1-H (Pr)], 3.04 [m, 1H, 3-H (Pr)], 3.15 [m, 1H, 2-H (Pr)], 3.18 [m, 1H, 1$\mathrm{H}(\mathrm{Pr})], 3.22$ [t, 2H, J= $7 \mathrm{~Hz}, 1-\mathrm{H}$ (ethyl)], 4.23 [m, 1H, $\alpha-\mathrm{H}$ (Lys)], 4.36 [d, 2H, J=6 Hz, $\mathrm{CH}_{2}$ (Bn)], 6.63 and 6.82 (2bs, 2H, HNCONH), 7.10-7.34 (m, 14H, aromatics), 8.07 [bs, 3H, $\delta$ $\mathrm{NH}_{3}{ }^{+}$(Lys)], 9.02 and 9.91 [2bs, 2H, $\alpha-\mathrm{NH}_{2}{ }^{+}$(Lys)], 9.48 (bs, 1H, NH-Bn); ${ }^{13} \mathrm{C} \mathrm{NMR} \mathrm{(75}$ MHz, DMSO-d $\left.\mathrm{d}_{6}\right) \delta 20.1\left(\mathrm{CH}_{2}, \mathrm{C}_{\gamma}\right), 26.7\left(\mathrm{CH}_{2}, \mathrm{C}_{\delta}\right), 31.8 .0\left(\mathrm{CH}_{2}, \mathrm{C}_{\beta}\right), 35.2\left(\mathrm{CH}_{2}, \mathrm{C}_{3}\right), 35.5$ $\left[\mathrm{CH}_{2}, \mathrm{C}_{2}\right.$ (ethyl)], $38.7\left[\mathrm{CH}_{2}, \mathrm{C}_{1}\right.$ (ethyl)], $39.1\left(\mathrm{CH}_{2}, \mathrm{C}_{\varepsilon}\right), 40.6\left(\mathrm{CH}_{2}, \mathrm{Bn}\right), 43.0\left(\mathrm{CH}_{2}, \mathrm{C}_{1}\right), 58.9$ $\left(\mathrm{CH}, \mathrm{C}_{2}\right), 60.4\left(\mathrm{CH}, \mathrm{C}_{\alpha}\right), 115.4-160.3(14 \mathrm{CH}$ and $4 \mathrm{C}$, aromatics), $162.2(\mathrm{HNCONH}), 167.9$ $(\mathrm{CONH})$; ES-MS $m / z[\mathrm{M}+1]^{+}$calcd for $\mathrm{C}_{31} \mathrm{H}_{40} \mathrm{FN}_{5} \mathrm{O}_{2}, 534.32$; found, 534.65 (100\%). 


\subsection{Synthesis of the indazole-containing protected ureas $(\boldsymbol{R})-$ and $(\boldsymbol{S})-\mathbf{2 4 b}$}

Diethyl amine $(0.22 \mathrm{~mL}, 2.1 \mathrm{mmol})$ was added to a solution of the corresponding Fmocprotected amine $(\boldsymbol{R})$ - and $(\boldsymbol{S}) \mathbf{- 1 2 b}(0.21 \mathrm{mmol})$ and the reaction mixture was stirred at $\mathrm{rt}$ for 2 h. Then, the solvent was evaporated to dryness and the residue was dissolved in EtOAc (50 $\mathrm{mL})$. The solution was successively washed with $\mathrm{H}_{2} \mathrm{O}(20 \mathrm{~mL})$ and brine $(20 \mathrm{~mL})$, dried over $\mathrm{Na}_{2} \mathrm{SO}_{4}$, and evaporated to dryness to give the deprotected amines $(\boldsymbol{R})$ - and $(\boldsymbol{S})-\mathbf{3 b}$, which were reserved. Apart, propylene oxide $(118 \mu \mathrm{L}, 1.68 \mathrm{mmol})$ was added to a $0{ }^{\circ} \mathrm{C}$ cooled solution of 1-(2,6-dichlorobenzyl)-3-(pyrrolidin-1-ylmethyl)-1H-indazole-6-amine [50] (22) (79 mg, $0.21 \mathrm{mmol})$ in dry THF $(5 \mathrm{~mL})$. Then, a solution of bis(trichloromethyl)carbonate (20 $\mathrm{mg}, 0.07 \mathrm{mmol})$ in dry THF $(1 \mathrm{~mL})$ was added dropwise and stirring was maintained at $0{ }^{\circ} \mathrm{C}$ for $15 \mathrm{~min}$. Afterwards, the mixture was added dropwise to a $0{ }^{\circ} \mathrm{C}$ cooled solution of $(\boldsymbol{R})$ - and (S)- 3b (98 mg, $0.21 \mathrm{mmol})$ in dry THF (5 mL) and stirred for $1 \mathrm{~h}$. Then, the reaction mixture was diluted with EtOAc $(50 \mathrm{~mL})$, washed successively with $\mathrm{H}_{2} \mathrm{O}(20 \mathrm{~mL})$ and brine $(20 \mathrm{~mL})$, dried over $\mathrm{Na}_{2} \mathrm{SO}_{4}$, and evaporated to dryness. The residue was purified by reversed phase chromatography, using $10-100 \% \mathrm{CH}_{3} \mathrm{CN}$ gradient in $0.05 \%$ TFA solution in $\mathrm{H}_{2} \mathrm{O}$ as mobile phase.

4.6.1. $\mathrm{N}_{\varepsilon}-$ Boc- $\mathrm{N}_{\alpha}-[(2 \mathrm{R})-1-(3-(1-(2,6-$ dichlorobenzyl)-3-(pyrrolidin-1-ylmethyl)-1H-indazol6-yl)ureido)-3-phenylpropan-2-yl]-lysine benzyl amide [(R)-24b]

Foam (36 mg, $20 \%$ ); HPLC $t_{\mathrm{R}} 16.33 \mathrm{~min}$; HPLC-MS $t_{\mathrm{R}} 3.26 \mathrm{~min} ;[\alpha]_{\mathrm{D}}{ }^{20}+5.6(c 1$, $\mathrm{MeOH}) ;{ }^{1} \mathrm{H}-\mathrm{RMN}\left(400 \mathrm{MHz}, \mathrm{CDCl}_{3}\right) \delta$ 1.02-1.46 [m, 4H, $\gamma$ - and $\delta$-H (Lys)], 1.31 (s, 9H, Boc), 1.69, 1.85, 3.02, 3.45 (4m, 8H, pyrrolidine), 1.75-1.94 [m, 2H, $\beta$-H (Lys)], 2.74-2.92 [m, 2H, $\varepsilon-\mathrm{H}(\mathrm{Lys})], 2.74-3.07$ [m, 2H, 3-H (Pr)], 3.27 [m, 1H, 2-H (Pr)], 3.21-3.43 [m, 2H, 1$\mathrm{H}(\mathrm{Pr})], 3.93$ [bs, 1H, $\alpha-\mathrm{H}$ (Lys)], 4.29 [d, J = $\left.7 \mathrm{~Hz}, 2 \mathrm{H}, \mathrm{CH}_{2}(\mathrm{Bn})\right], 4.36$ (s, 2H, $\mathrm{CH}_{2^{-}}$ pyrrolidine), 4.64 (s, $1 \mathrm{H}, \mathrm{NH}$-Boc), 5.52 (s, 2H, $\mathrm{CH}_{2}$-diClPh), 6.94 [d, J = $7 \mathrm{~Hz}, 1 \mathrm{H}, 5-\mathrm{H}$ (indazole)], 7.36-7.00 (m, 13H, Ph ), 7.41 [d, J = 7 Hz, 1H, 4-H (indazole)], 7.87 [s, 1H, 7-H (indazole)], 8.12 (s, $1 \mathrm{H}, \mathrm{NH}-\mathrm{Bn}), 8.72$ and 11.8 (2bs, 2H, HNCONH); ${ }^{13} \mathrm{C}-\mathrm{RMN}(100 \mathrm{MHz}$, $\left.\mathrm{CDCl}_{3}\right) 21.7\left(\mathrm{CH}_{2}, \mathrm{C}_{\gamma}\right), 23.4\left(\mathrm{CH}_{2}\right.$, pyrrolidine $), 28.3\left(\mathrm{CH}_{3}, \mathrm{Boc}\right), 29.1\left(\mathrm{CH}_{2}, \mathrm{C}_{\delta}\right), 29.5\left(\mathrm{CH}_{2}\right.$, $\left.\mathrm{C}_{\beta}\right), 34.9\left(\mathrm{CH}_{2}, \mathrm{C}_{3}\right), 40.8\left(\mathrm{CH}_{2}, \mathrm{C}_{1}\right), 43.8\left(\mathrm{CH}_{2}, \mathrm{Bn}\right), 47.7\left(\mathrm{CH}_{2}-\mathrm{diClPh}\right), 48.1,52.3\left(\mathrm{CH}_{2}\right.$, pyrrolidine and $\mathrm{CH}_{2}$-pyrrolidine), $59.3\left(\mathrm{CH}, \mathrm{C}_{\alpha}\right), 60.2\left(\mathrm{CH}, \mathrm{C}_{2}\right), 79.5(\mathrm{C}, \mathrm{Boc}), 98.8\left[\mathrm{C}_{7}\right.$

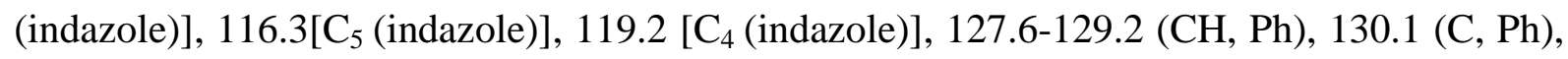
$131.2(\mathrm{C}, \mathrm{diClPh}), 133.7\left[\mathrm{C}_{5}\right.$ (indazole) $], 136.8(\mathrm{C}, \mathrm{Bn}), 137.3(2 \mathrm{C}, \mathrm{diClPh}), 138.8\left[\mathrm{C}_{6}\right.$ 
(indazole)], 141.6 [C $\mathrm{C}_{7 \mathrm{a}}$ (indazole)], 157.42 (HNCONH), $162.2(\mathrm{CO}, \mathrm{Boc}), 167.2$ (CONH); ES-MS m/z $[\mathrm{M}+1]^{+}$calcd. for $\mathrm{C}_{47} \mathrm{H}_{58} \mathrm{Cl}_{2} \mathrm{~N}_{8} \mathrm{O}_{4}, 869.40$; found, 435.29 (100\%) [(M+2)/2 $]^{+}$, $869.49(6 \%)[\mathrm{M}+1]^{+}$. Anal. calcd. for $\mathrm{C}_{47} \mathrm{H}_{58} \mathrm{Cl}_{2} \mathrm{~N}_{8} \mathrm{O}_{4}$ : C, 64.89; H, 6.72; N, 12.88; Found: C, 64.95; H, 6.82; N, 12.95.

4.6.2. $\quad \mathrm{N}_{\varepsilon}-$ Boc $-\mathrm{N}_{\alpha}-[(2 \mathrm{~S})-1-(3-(1-(2,6-d i c h l o r o b e n z y l)-3-(p y r r o l i d i n-1-y l m e t h y l)-1 H$-indazol6-yl)ureido)-3-phenylpropan-2-yl]-lysine benzyl amide $[(\mathbf{S})-\mathbf{2 4 b}]$

Foam (155 mg, 85\%); HPLC $t_{\mathrm{R}} 16.51 \mathrm{~min}$; HPLC-MS $t_{\mathrm{R}} 3.26 \mathrm{~min} ;[\alpha]_{\mathrm{D}}{ }^{20}+8.4(c 1$, $\mathrm{MeOH}) ;{ }^{1} \mathrm{H}-\mathrm{RMN}\left(500 \mathrm{MHz}, \mathrm{CDCl}_{3}\right) \delta$ 1.08-1.42 [m, 4H, $\gamma$ - and $\delta$-H (Lys)], 1.29 (s, 9H, Boc), 1.70, 1.87, 2.98, 3.45 (4m, 8H, pyrrolidine), 1.76-1.83 [m, 2H, $\beta-\mathrm{H}$ (Lys)], 2.72-2.86 [m, 2H, ع- H (Lys)], 2.74-3.07 [m, 2H, 3-H (Pr)], 3.23 [m, 1H, 2-H (Pr)], 3.18-3.31 [m, 1H, 1$\mathrm{H}(\mathrm{Pr})], 3.55$ [m, 1H, 1-H (Pr)], 4.25 [bs, 1H, $\alpha-\mathrm{H}(\mathrm{Lys})], 4.30$ [dd, J = 5 and $15 \mathrm{~Hz}, 2 \mathrm{H}, \mathrm{CH}_{2}$ (Bn)], 4.36 (s, 2H, $\mathrm{CH}_{2}$-pyrrolidine), 4.70 (s, 1H, $\mathrm{NH}$-Boc), 5.50 (s, 2H, $\mathrm{CH}_{2}$-diClPh), 6.99 [d, $\mathrm{J}=7 \mathrm{~Hz}, 1 \mathrm{H}, 5-\mathrm{H}$ (indazole)], 7.09-7.34 (m, 13H, Ph), 7.43 [d, J = 7 Hz, 1H, 4-H (indazole)], 7.80 [s, 1H, 7-H (indazole)], 9.18 (s, 1H, NH-Bn), 8.92 and 11.78 (2bs, 2H, HNCONH); ${ }^{13} \mathrm{C}-$ RMN (125 MHz, $\left.\mathrm{CDCl}_{3}\right) \delta 21.9\left(\mathrm{CH}_{2}, \mathrm{C}_{\gamma}\right), 23.6\left(\mathrm{CH}_{2}\right.$, pyrrolidine $), 28.5\left(\mathrm{CH}_{3}, \mathrm{Boc}\right), 29.3$ $\left(\mathrm{CH}_{2}, \mathrm{C}_{\delta}\right), 30.7\left(\mathrm{CH}_{2}, \mathrm{C}_{\beta}\right), 30.7\left(\mathrm{CH}_{2}, \mathrm{C}_{\beta}\right), 36.6\left(\mathrm{CH}_{2}, \mathrm{C}_{3}\right), 39.7\left(\mathrm{CH}_{2}, \mathrm{C}_{\varepsilon}\right), 40.3\left(\mathrm{CH}_{2}, \mathrm{C}_{1}\right)$, $44.2\left(\mathrm{CH}_{2}, \mathrm{Bn}\right), 48.0\left(\mathrm{CH}_{2}\right.$-diClPh$), 48.6\left(\mathrm{CH}_{2}\right.$-pyrrolidine $), 52.8\left(\mathrm{CH}_{2}\right.$, pyrrolidine $), 60.6$

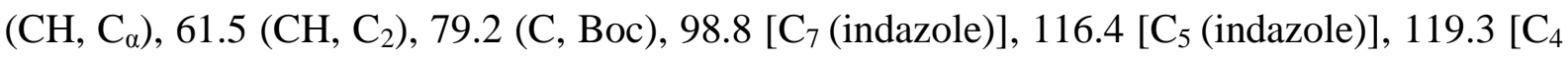
(indazole)], 119.8 [C $\mathrm{C}_{3 \mathrm{a}}$ (indazole)], $167.6(\mathrm{CONH}), 127.8-129.5(\mathrm{CH}, \mathrm{Ph}), 130.5(\mathrm{C}, \mathrm{Ph})$, $131.6(\mathrm{C}, \mathrm{diClPh}), 133.9$ [C $\mathrm{C}_{5}$ (indazole) $], 134.3(\mathrm{C}, \mathrm{Bn}), 137.0(2 \mathrm{C}, \mathrm{diClPh}), 138.8\left[\mathrm{C}_{6}\right.$ (indazole)], $141.7\left[\mathrm{C}_{7 \mathrm{a}}\right.$ (indazole)], $158.5(\mathrm{C}, \mathrm{HNCONH}), 162.1(\mathrm{CO}, \mathrm{Boc}), 167.6(\mathrm{CONH})$; ES-MS m/z $[\mathrm{M}+1]^{+}$calcd. for $\mathrm{C}_{47} \mathrm{H}_{58} \mathrm{Cl}_{2} \mathrm{~N}_{8} \mathrm{O}_{4}, 869.40$; found, 435.29 (100\%) [(M+2)/2 $]^{+}$, $869.49(5 \%)[\mathrm{M}+1]^{+}$; Anal. calcd. for $\mathrm{C}_{47} \mathrm{H}_{58} \mathrm{Cl}_{2} \mathrm{~N}_{8} \mathrm{O}_{4}$ : C, 64.89; H, 6.72; N, 12.88; Found: C, 65.02; H, 6.85; N, 12.92.

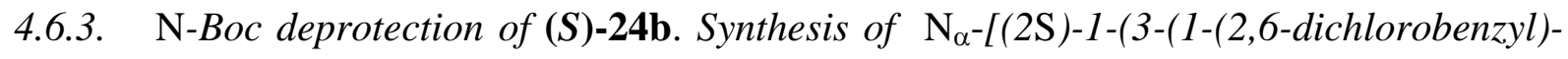
3-(pyrrolidin-1-ylmethyl)-1H-indazol-6-yl)ureido)-3-phenylpropan-2-yl]-lysine benzyl amide trihydrochloride $[(\boldsymbol{S})-\mathbf{2 5 b}]$

The protected urea $(\boldsymbol{S}) \mathbf{- 2 4 b}(130 \mathrm{mg}, 0.15 \mathrm{mmol})$ was dissolved in a $3 \mathrm{M}$ solution of $\mathrm{HCl}$ in EtOAc $(2 \mathrm{~mL})$ and stirred at $\mathrm{rt}$ for $2 \mathrm{~h}$. Then, the solvent was evaporated under reduced pressure, the residue was dissolved in $\mathrm{H}_{2} \mathrm{O}(3 \mathrm{~mL})$ and the solution was lyophilized to give the urea $(S)-25 b(131 \mathrm{mg}, 100 \%)$. HPLC $t_{\mathrm{R}} 13.11 \mathrm{~min}$; HPLC-MS $t_{\mathrm{R}} 2.73 \mathrm{~min} ;[\alpha]_{\mathrm{D}}{ }^{20}+1.6(c$ 
1.2, MeOH); ${ }^{1} \mathrm{H}-\mathrm{RMN}\left(500 \mathrm{MHz}, \mathrm{DMSO}-\mathrm{d}_{6}\right) \delta(\mathrm{ppm}): 1.32$ [m, 2H, $\left.\gamma-\mathrm{H}(\mathrm{Lys})\right], 1.53$ [m, 2H, $\delta$-H (Lys)], 1.74 [m, 2H, $\beta-\mathrm{H}$ (Lys)], 1.77-1.95 [m, 4H, 2 $\mathrm{CH}_{2}$ (pyrrolidine)], 2.66 [m, 2H, $\varepsilon-\mathrm{H}$ (Lys)], 2.84 [dd, 1H, J = 10 and $13 \mathrm{~Hz}, 3-\mathrm{H}(\mathrm{Pr})], 3.04$ [m, 4H, 2CH $\mathrm{CH}_{2}$ (pyrrolidine)], 3.08$3.16[\mathrm{~m}, 1 \mathrm{H}, 3-\mathrm{H}(\mathrm{Pr})], 3.12$ [m, 1H, 2-H (Pr)], 3.19-3.59 [m, 2H, 1-H (Pr)], 4.14 [m, 1H, $\alpha-\mathrm{H}$ (Lys)], 4.32 [t, 2H, J = $\left.7 \mathrm{~Hz}, \mathrm{CH}_{2}(\mathrm{Bn})\right], 4.54$ (s, 2H, $\mathrm{CH}_{2}$-pyrrolidine), 5.57 (s, 2H, $\mathrm{CH}_{2^{-}}$ diClPh), 7.02-7.95 (m, 16H, aromatics), 8.01 and 9.66 (2s, 2H, HNCONH), 9.08 (s, 1H, NHBn), 9.43 and 10.58 [2bs, $\left.2 \mathrm{H}, \alpha-\mathrm{NH}_{2}{ }^{+}(\mathrm{Lys})\right] \cdot{ }^{13} \mathrm{C}-\mathrm{RMN}\left(125 \mathrm{MHz}, \mathrm{DMSO}-\mathrm{d}_{6}\right) \delta$ (ppm): 21.4 $\left(\mathrm{CH}_{2}, \mathrm{C}_{\gamma}\right), 22.6\left(2 \mathrm{CH}_{2}\right.$, pyrrolidine $), 26.4\left(\mathrm{CH}_{2}, \mathrm{C}_{\delta}\right), 29.7\left(\mathrm{CH}_{2}, \mathrm{C}_{\beta}\right), 35.1\left(\mathrm{CH}_{2}, \mathrm{C}_{3}\right), 38.2$ $\left(\mathrm{CH}_{2}, \mathrm{C}_{\varepsilon}\right), 38.3\left(\mathrm{CH}_{2}, \mathrm{C}_{1}\right), 42.6\left(\mathrm{CH}_{2}, \mathrm{Bn}\right), 47.4\left(\mathrm{CH}_{2}\right.$, diClPh), $47.7\left(\mathrm{CH}_{2}, \mathrm{CH}_{2}\right.$-pyrrolidine $)$, $52.7\left(2 \mathrm{CH}_{2}\right.$, pyrrolidine), $58.2\left(\mathrm{CH}, \mathrm{C}_{\alpha}\right), 58.8\left(\mathrm{CH}, \mathrm{C}_{2}\right), 98.4\left[\mathrm{C}_{7}\right.$ (indazole) $), 114.6\left[\mathrm{C}_{5}\right.$

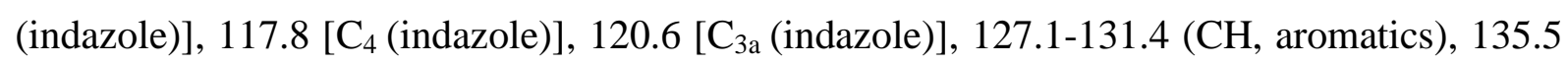
(C, Bn), 136.1 (C, diClPh), $136.2(\mathrm{C}, \mathrm{Ph}), 138.2$ [C $\mathrm{C}_{5}$ (indazole)], 138.4 (2C, diClPh), 139.7 $\left[\mathrm{C}_{6}\right.$ (indazole)], $141.3\left[\mathrm{C}_{7 \mathrm{a}}\right.$ (indazole)], $156.1(\mathrm{HNCONH}), 167.3(\mathrm{CONH}) ; \mathrm{ES}-\mathrm{MS} \mathrm{m} / \mathrm{z}$ $[\mathrm{M}+1]^{+}$calcd for $\mathrm{C}_{42} \mathrm{H}_{50} \mathrm{Cl}_{2} \mathrm{~N}_{8} \mathrm{O}_{2}, 769.34$; found, $385.28(60 \%)[(\mathrm{M}+2) / 2]^{+}, 769.37(6 \%)$ $[\mathrm{M}+1]^{+}$.

\subsection{General procedure for the synthesis of thioureas $(\boldsymbol{R})-$ and $(\boldsymbol{S})-\mathbf{2 6} \mathbf{a}$}

Diethyl amine $(0.32 \mathrm{~mL}, 3 \mathrm{mmol})$ was added to a solution of the corresponding Fmocprotected amine $(\boldsymbol{R})$ - and $(\boldsymbol{S})$-12a $(0.3 \mathrm{mmol})$ and the reaction mixture was stirred at $\mathrm{rt}$ for $2 \mathrm{~h}$. Then, the solvent was evaporated to dryness and the residue was dissolved in EtOAc $(50 \mathrm{~mL})$. The solution was successively washed with $\mathrm{H}_{2} \mathrm{O}(20 \mathrm{~mL})$ and brine $(20 \mathrm{~mL})$, dried over $\mathrm{Na}_{2} \mathrm{SO}_{4}$, and evaporated to dryness to give the deprotected amines $(\boldsymbol{R})$ - and $(\boldsymbol{S})-\mathbf{3 a}$. These amines were dissolved in dry $\mathrm{CH}_{2} \mathrm{Cl}_{2}(10 \mathrm{~mL})$ and the solution was cooled at $0^{\circ} \mathrm{C}$. Phenyl isothiocyanate $(40.5 \mathrm{mg}, 0.3 \mathrm{mmol})$ was added and the mixture was stirred for $2 \mathrm{~h}$. Then, the solvent was evaporated and the residue was dissolved in EtOAc $(50 \mathrm{~mL})$. This solution was successively washed with $\mathrm{H}_{2} \mathrm{O}(20 \mathrm{~mL})$ and brine $(20 \mathrm{~mL})$, dried over $\mathrm{Na}_{2} \mathrm{SO}_{4}$, and evaporated to dryness. The residue was purified by flash chromatography, using 20-100\% EtOAc gradient in hexane as eluant to give the corresponding thiourea $(\boldsymbol{R})$ - and $(\boldsymbol{S}) \mathbf{- 2 6}$.

4.7.1. $\mathrm{N}_{\delta}$-Boc- $\mathrm{N}_{\alpha}-[(2 \mathrm{R})-3-P h e n y l-1-(3-p h e n y l t h i o u r e i d o) p r o p a n-2-y l]-o r n i t h i n e \quad b e n z y l$ amide $[(\boldsymbol{R})-\mathbf{2 6 a}]$

Foam $(121 \mathrm{mg}, 68 \%)$; HPLC $t_{\mathrm{R}} 18.42 \mathrm{~min} ;[\alpha]_{\mathrm{D}}{ }^{20}+3.0(c 1, \mathrm{MeOH}) ;{ }^{1} \mathrm{H}$ NMR $(500 \mathrm{MHz}$, $\left.\mathrm{CDCl}_{3}\right) \delta 1.38(\mathrm{~m}, 9 \mathrm{H}, \mathrm{Boc}), 1.10-1.65[\mathrm{~m}, 4 \mathrm{H}, \beta-\mathrm{H}$ and $\gamma-\mathrm{H}(\mathrm{Orn})], 2.71-2.91[\mathrm{~m}, 3 \mathrm{H}, 3-\mathrm{H}$ 
(Pr) and $\delta-\mathrm{H}(\mathrm{Orn})], 2.97[\mathrm{dd}, 1 \mathrm{H}, \mathrm{J}=7.5$ and 13Hz, 3-H (Pr)], 3.09-3.30 [m, 2H, $\alpha-\mathrm{H}(\mathrm{Orn})$ and 2-H (Pr)], $3.63[\mathrm{~d}, 2 \mathrm{H}, \mathrm{J}=5 \mathrm{~Hz}, 1-\mathrm{H}(\mathrm{Pr})], 4.21$ [dd, $\mathrm{J}=6.5$ and $\left.15 \mathrm{~Hz}, 1 \mathrm{H}, \mathrm{CH}_{2}(\mathrm{Bn})\right]$, $4.43\left[\mathrm{dd}, 1 \mathrm{H}, \mathrm{J}=5\right.$ and $\left.15 \mathrm{~Hz}, \mathrm{CH}_{2}(\mathrm{Bn})\right], 4.53$ (s, 1H, NH-Boc), 6.79 and 7.85 (2s, 2H, $\mathrm{HnCSNH}), 7.04-7.63$ (m, 16H, aromatics and $\mathrm{NH}-\mathrm{Bn}) ;{ }^{13} \mathrm{C} \mathrm{NMR}\left(125 \mathrm{MHz}, \mathrm{CDCl}_{3}\right) \delta 25.9$ $\left(\mathrm{CH}_{2}, \mathrm{C}_{\gamma}\right), 28.3\left(\mathrm{CH}_{3}, \mathrm{Boc}\right), 30.5\left(\mathrm{CH}_{2}, \mathrm{C}_{\beta}\right), 39.4\left(\mathrm{CH}_{2}, \mathrm{C}_{\delta}\right), 43.2\left(\mathrm{CH}_{2}, \mathrm{Bn}\right), 58.1\left(\mathrm{CH}, \mathrm{C}_{\alpha}\right)$, $59.7\left(\mathrm{CH}, \mathrm{C}_{2}\right), 79.3$ (C, Boc), 125.0-138.3 (15 CH and $3 \mathrm{C}$, aromatics), 156.3 (CO, Boc), $174.9(\mathrm{CONH}), 181.2(\mathrm{CS})$; ES-MS m/z $[\mathrm{M}+1]^{+}$calcd. for $\mathrm{C}_{33} \mathrm{H}_{44} \mathrm{~N}_{5} \mathrm{O}_{3} \mathrm{~S}, 590.31$; found, 590.51 (100 \%). Anal. calcd. for $\mathrm{C}_{33} \mathrm{H}_{43} \mathrm{~N}_{5} \mathrm{O}_{3} \mathrm{~S}$ : C, 67.20; H, 7.35; N, 11.87; Found: C, 67.24; $\mathrm{H}, 7.55 ; \mathrm{N}, 11.93$.

4.7.2. $\mathrm{N}_{\delta}$-Boc- $\mathrm{N}_{\alpha}$-[(2S)-3-Phenyl-1-(3-phenylthioureido)propan-2-yl]-ornithine benzyl amide $[(\mathbf{S})-\mathbf{2 6 a}]$

Foam $(127 \mathrm{mg}, 72 \%)$; HPLC $t_{\mathrm{R}} 18.67 \mathrm{~min} ;[\alpha]_{\mathrm{D}}{ }^{20}+7.5\left(c\right.$ 1.1, MeOH); ${ }^{1} \mathrm{H}$ NMR (500 $\left.\mathrm{MHz}, \mathrm{CDCl}_{3}\right) \delta 1.38$ (s, 9H, Boc), 1.23-1.90 [m, 4H, $\beta-\mathrm{H}$ and $\left.\gamma-\mathrm{H}(\mathrm{Orn})\right], 2.87-3.17$ [m, 6H, 1- and 3-H (Pr), and $\delta$-H (Orn)], 3.40 [m, 1H, 2-H (Pr)], 3.62 [m,1H, $\alpha-\mathrm{H}(\mathrm{Orn})], 4.10$ [dd, J = 7 and $\left.15 \mathrm{~Hz}, 1 \mathrm{H}, \mathrm{CH}_{2}(\mathrm{Bn})\right], 4.38$ [dd, $\mathrm{J}=5$ and $\left.15 \mathrm{~Hz}, 1 \mathrm{H}, \mathrm{CH}_{2}(\mathrm{Bn})\right], 4.71$ (s, 1H, NH-Boc), 6.69 and 8.05 (2s, 2H, HNCSNH), 7.02-7.54 (m, 16H, aromatics and NH-Bn); ${ }^{13} \mathrm{C}$ NMR (125 $\left.\mathrm{MHz}, \mathrm{CDCl}_{3}\right) \delta 26.2\left(\mathrm{CH}_{2}, \mathrm{C}_{\gamma}\right), 28.4\left(\mathrm{CH}_{3}, \mathrm{Boc}\right), 29.3\left(\mathrm{CH}_{2}, \mathrm{C}_{\beta}\right), 39.5\left(\mathrm{CH}_{2}, \mathrm{C}_{\delta}\right), 40.5\left(\mathrm{CH}_{2}\right.$, $\left.\mathrm{C}_{3}\right), 43.1\left(\mathrm{CH}_{2}, \mathrm{Bn}\right), 58.3\left(\mathrm{CH}, \mathrm{C}_{\alpha}\right), 59.3\left(\mathrm{CH}, \mathrm{C}_{2}\right), 79.5(\mathrm{C}, \mathrm{Boc}), 124.5-138.4(15 \mathrm{CH}$ and $3 \mathrm{C}$, aromatics), 156.4 (CO, Boc), $173.4(\mathrm{CONH}), 181.3$ (CS); ES-MS m/z [M+1] ${ }^{+}$calcd. for $\mathrm{C}_{33} \mathrm{H}_{43} \mathrm{~N}_{5} \mathrm{O}_{3} \mathrm{~S}$, 590.31; found, 590.58 (100\%). Anal. calcd. for $\mathrm{C}_{33} \mathrm{H}_{43} \mathrm{~N}_{5} \mathrm{O}_{3} \mathrm{~S}: \mathrm{C}, 67.20 ; \mathrm{H}$, 7.35; N, 11.87; Found: C, 67.15; H, 7.45; N, 11.86.

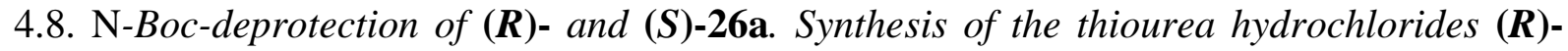
and $(S)-27 a$

It was carried out by applying the above described methodology for $N$-Boc removal in the urea analogues.

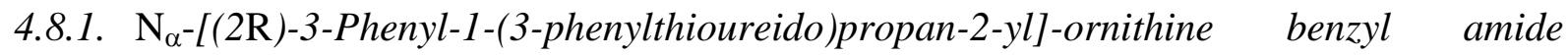
dihydrochloride $[(\boldsymbol{R})-\mathbf{2 7} \mathbf{a}]$

Amorphous solid (56 mg, 100\%); HPLC $t_{\mathrm{R}} 13.29 \mathrm{~min} ;[\alpha]_{\mathrm{D}}{ }^{20}+10.7$ (c 1, MeOH); ${ }^{1} \mathrm{H}$ NMR (500 MHz, DMSO-d $\left.{ }_{6}\right) \delta$ 1.50-1.76 [m, 2H, $\gamma-\mathrm{H}($ Orn) ], 1.78-2.06 [m, 2H, $\beta-\mathrm{H}$ (Orn)], 2.72$3.27[\mathrm{~m}, 4 \mathrm{H}, 3-\mathrm{H}(\mathrm{Pr})$ and $\delta-\mathrm{H}(\mathrm{Orn})], 3.46-3.66$ [m, 2H, 1-H (Pr)], 3.80 [m, 1H, 2-H (Pr)], 
$4.25\left[\mathrm{dd}, 1 \mathrm{H}, \mathrm{J}=6\right.$ and $\left.15 \mathrm{~Hz}, \mathrm{CH}_{2}(\mathrm{Bn})\right], 4.37$ [m, 1H, $\left.\alpha-\mathrm{H}(\mathrm{Orn})\right], 4.53$ [dd, 1H, J = 5 and 15 $\left.\mathrm{Hz}, \mathrm{CH}_{2}(\mathrm{Bn})\right], 7.10$ (s,1H, HNCSNH), 6.93-7.55(m, 16H, aromatics and HNCSNH), 8.05 [bs, $3 \mathrm{H}, \delta-\mathrm{NH}_{3}{ }^{+}$(Orn)], 8.75 and 10.22 [2bs, $2 \mathrm{H}, \alpha-\mathrm{NH}_{2}{ }^{+}$(Orn)], 9.41(s, $\left.1 \mathrm{H}, N H-\mathrm{Bn}\right) ;{ }^{13} \mathrm{C} \mathrm{NMR}$ $\left(125 \mathrm{MHz}, \mathrm{DMSO}-\mathrm{d}_{6}\right) \delta 22.8\left(\mathrm{CH}_{2}, \mathrm{C}_{\gamma}\right), 27.1\left(\mathrm{CH}_{2}, \mathrm{C}_{\beta}\right), 28.2\left(\mathrm{CH}_{2}, \mathrm{C}_{3}\right), 34.1\left(\mathrm{CH}_{2}, \mathrm{C}_{\delta}\right), 38.1$ $\left(\mathrm{CH}_{2}, \mathrm{Bn}\right), 42.7\left(\mathrm{CH}_{2}, \mathrm{C}_{1}\right), 57.0\left(\mathrm{CH}, \mathrm{C}_{2}\right), 58.3\left(\mathrm{CH}, \mathrm{C}_{\alpha}\right), 117.7-139.9(15 \mathrm{CH}$ and $3 \mathrm{C}$, aromatics), $166.9(\mathrm{CONH}), 181.0(\mathrm{CS})$; ES-MS m/z [M+1] ${ }^{+}$calcd. for $\mathrm{C}_{28} \mathrm{H}_{35} \mathrm{~N}_{5} \mathrm{OS}, 490.26$; found $246.13(100 \%)[(\mathrm{M}+2) / 2]^{+}, 490.48(15 \%)[\mathrm{M}+1]^{+}$.

4.8.2. $\mathrm{N}_{\alpha}$-[(2S)-3-Phenyl-1-(3-phenylthioureido)propan-2-yl]-ornithine benzyl amide dihydrochloride $[(\boldsymbol{S})-\mathbf{2 7} \mathbf{a}]$

Amorphous solid (54 mg, 100\%); HPLC $t_{\mathrm{R}} 13.40 \mathrm{~min} ;[\alpha]_{\mathrm{D}}{ }^{20}+2.9(c 0.7, \mathrm{MeOH}) ;{ }^{1} \mathrm{H}$ NMR (500 MHz, DMSO-d $\left.{ }_{6}\right) \delta$ 1.51-2.06 [m, 4H, $\beta$ - and $\gamma-\mathrm{H}($ Orn)], 2.73-3.22 [m, 5H, 2- and 3-H (Pr), and $\delta-\mathrm{H}(\mathrm{Orn})], 3.52-3.60$ [m, 2H, 1-H (Pr)], 4.29 [m, 1H, $\alpha-\mathrm{H}(\mathrm{Orn})], 4.40$ [d, 2H, J $=6 \mathrm{~Hz}, \mathrm{CH}_{2}(\mathrm{Bn}], 7.10(\mathrm{~s}, 1 \mathrm{H}, \mathrm{HNCSNH}), 6.69-7.71(\mathrm{~m}, 15 \mathrm{H}$, aromatics and HNCSNH), $7.94\left[\mathrm{bs}, 3 \mathrm{H}, \delta-\mathrm{NH}_{3}{ }^{+}(\mathrm{Orn})\right], 9.42$ (s, $\left.1 \mathrm{H}, \mathrm{NH}-\mathrm{Bn}\right), 9.19$ and 9.54 [2bs, $\left.2 \mathrm{H}, \alpha-\mathrm{NH}_{2}{ }^{+}(\mathrm{Orn})\right] ;{ }^{13} \mathrm{C}$ NMR (125 MHz, DMSO-d $)_{6} \delta 22.7\left(\mathrm{CH}_{2}, \mathrm{C}_{\gamma}\right), 27.1\left(\mathrm{CH}_{2}, \mathrm{C}_{\beta}\right), 31.6\left(\mathrm{CH}_{2}, \mathrm{C}_{3}\right), 38.3\left(\mathrm{CH}_{2}, \mathrm{C}_{\delta}\right)$, $38.9\left(\mathrm{CH}_{2}, \mathrm{Bn}\right), 42.8\left(\mathrm{CH}_{2}, \mathrm{C}_{1}\right), 57.0\left(\mathrm{CH}, \mathrm{C}_{2}\right), 58.8\left(\mathrm{CH}, \mathrm{C}_{\alpha}\right), 123.4-138.7(15 \mathrm{CH}$ and $3 \mathrm{C}$, aromatics), 166.9 (CONH), 181.7 (CS); ES-MS m/z [M+1] ${ }^{+}$calcd. for $\mathrm{C}_{28} \mathrm{H}_{35} \mathrm{~N}_{5} \mathrm{OS}, 490.26$; found $246.13(100 \%)[(\mathrm{M}+2) / 2]^{+}, 490.48(15 \%)[\mathrm{M}+1]^{+}$.

\subsection{Platelet aggregation inhibition assay}

Whole blood was obtained from human volunteers who were not taking any platelet altering drugs for two weeks prior to donation. Blood was collected by venous puncture into $2.7 \mathrm{~mL}$ vacutainer tubes containing $3.2 \%$ buffered sodium citrate. Blood was centrifuged at $250 \times \mathrm{g}$ for $7 \mathrm{~min}$ to obtain platelet rich plasma (PRP). After removal of PRP, the blood was recentrifuged at $900 \times \mathrm{g}$ for $10 \mathrm{~min}$ to obtain platelet poor plasma (PPP). The PPP was used as a reference in the optical aggregation and as a diluent to achieve a final platelet concentration of 200.000 platelet $/ \mu \mathrm{L}$ in PRP. Tests were performed in an optical aggregometer (Chrono-Log Model 440 Four Channel). Briefly, a $0.5 \mathrm{~mL}$ sample of diluted PRP was added to a glass cuvette and incubated with either vehicle (DMSO solution) or tested compound, at a 0.1 $\mathrm{mg} / \mathrm{mL}$ concentration, for $5 \mathrm{~min}$ at $37^{\circ} \mathrm{C}$. At the beginning of each experiment, aggregation response to SFLLRN $(30 \mu \mathrm{M})$ was evaluated and the maximum aggregation value at the end of 5 min was recorded. Aggregation response to SFLLRN plus compound was recorded and 
compared to control (SFLLRN/vehicle) to determine the \% of inhibition. Each compound was tested twice and the results are the mean of the two assays.

\section{Acknowledgements}

This work was supported by the Spanish Ministerio de Ciencia e Innovación grant SAF2009-09323. P. V.-A. held a FPI fellowship from the Ministerio de Ciencia e Innovación.

\section{References}

[1] E. Di Cera, Thrombin, Mol. Aspects Med., 29 (2008) 203-254.

[2] S.R. Macfarlane, M.J. Seatter, T. Kanke, G.D. Hunter, R. Plevin, Proteinase-activated receptors, Pharmacol. Rev., 53 (2001) 245-282.

[3] L. Martorell, J. Martínez-González, C. Rodríguez, M. Gentile, O. Calvayrac, L. Badimon, Thrombin and protease-activated receptors (PARs) in atherothrombosis, Thromb. Haemost., 99 (2008) 305-315.

[4] V.S. Ossovskaya, N.W. Bunnett, Protease-activated receptors: contribution to physiology and disease, Physiol. Rev., 84 (2004) 579-621.

[5] E. Sokolova, G. Reiser, Prothrombin/thrombin and the thrombin receptors PAR-1 and PAR-4 in the brain: localization, expression and participation in neurodegenerative diseases, Thromb. Haemost., 100 (2008) 576-581.

[6] W. Luo, Y. Wang, G. Reiser, The role of thrombin and thrombin receptors in the brain, in: M.E. Maragoudakis, N.E. Tsopanoglou (Eds.) Thrombin: Physiology and Disease, Springer, New York, 2009, pp. 133-159.

[7] Y. Hashimotodani, T. Ohno-Shosaku, M. Yamazaki, K. Sakimura, M. Kano, Neuronal proteaseactivated receptor 1 drives synaptic retrograde signaling mediated by the endocannabinoid 2arachidonoylglycerol, J. Neurosci., 31 (2011) 3104-3109.

[8] M. Steinhoff, J. Buddenkotte, V. Shpacovitch, A. Rattenholl, C. Moormann, N. Vergnolle, T.A. Luger, M.D. Hollenberg, Proteinase-activated receptors: transducers of proteinase-mediated signaling in inflammation and immune response, Endocr. Rev., 26 (2005) 1-43.

[9] R. Ramachandran, M. El-Daly, M. Saifeddine, M.D. Hollenberg, Thrombin: To PAR or not to PAR, and the regulation of inflamation, in: M.E. Maragoudakis, N.E. Tsopanoglou (Eds.) Thrombin: Physiology and Disease, Springer, New York, 2009, pp. 19-46.

[10] C.N. Pagel, S.J. Song, L.H. Loh, E.M. Tudor, T.A. Murray-Rust, R.N. Pike, E.J. Mackie, Thrombin-stimulated growth factor and cytokine expression in osteoblasts is mediated by protease-activated receptor-1 and prostanoids, Bone, 44 (2009) 813-821.

[11] P. Arora, T.K. Ricks, J. Trejo, Protease-activated receptor signalling, endocytic sorting and dysregulation in cancer, J. Cell Sci., 120 (2007) 921-928.

[12] E. Camerer, Protease signaling in tumor progression, Thromb. Res., 120 Suppl 2 (2007) S75S81.

[13] K.S. Borensztajn, C.A. Spek, Protease-activated receptors, apoptosis and tumor growth, Pathophysiol. Haemost. Thromb., 36 (2008) 137-147.

[14] B. Kobrinsky, S. Karpatkin, The role of thrombin in tumor biology, in: M.E. Maragoudakis, N.E. Tsopanoglou (Eds.) Thrombin: Physiology and Disease, Springer, New York, 2009, pp. 161-172.

[15] M.T. García-López, M. Gutiérrez-Rodríguez, R. Herranz, Thrombin-Activated Receptors: Promising Targets for Cancer Therapy?, Curr Med Chem, 17 (2010) 109-128.

[16] S.R. Coughlin, Thrombin signalling and protease-activated receptors, Nature, 407 (2000) 258264. 
[17] J. Trejo, Regulation of thrombin receptor signaling, in: M.E. Maragoudakis, N.E. Tsopanoglou (Eds.) Thrombin: Physiology and Disease, Springer, New York, 2009, pp. 47-61.

[18] R. Shah, Protease-activated receptors in cardiovascular health and diseases, Am. Heart J., 157 (2009) 253-262.

[19] C. Chen, B.E. Maryanoff, P. Andrade-Gordon, Thrombin receptor modulators: Medicinal chemistry, biological evaluation, and clinical applications, in: M.E. Maragoudakis, N.E. Tsopanoglou (Eds.) Thrombin: Physiology and Disease, Springer, New York, 2009, pp. 205236.

[20] C. Tellez, M. Bar-Eli, Role and regulation of the thrombin receptor (PAR-1) in human melanoma, Oncogene, 22 (2003) 3130-3137.

[21] S. Granovsky-Grisaru, S. Zaidoun, D. Grisaru, Y. Yekel, D. Prus, U. Beller, R. Bar-Shavit, The pattern of Protease Activated Receptor 1 (PAR1) expression in endometrial carcinoma, Gynecol. Oncol., 103 (2006) 802-806.

[22] X. Zhang, W. Wang, L.D. True, R.L. Vessella, T.K. Takayama, Protease-activated receptor-1 is upregulated in reactive stroma of primary prostate cancer and bone metastasis, Prostate, 69 (2009) 727-736.

[23] C. Rudroff, S. Seibold, R. Kaufmann, C.C. Zetina, K. Reise, U. Schafer, A. Schneider, M. Brockmann, J. Scheele, E.A. Neugebauer, Expression of the thrombin receptor PAR-1 correlates with tumour cell differentiation of pancreatic adenocarcinoma in vitro, Clin. Exp. Metastasis, 19 (2002) 181-189.

[24] S. Grisaru-Granovsky, Z. Salah, M. Maoz, D. Pruss, U. Beller, R. Bar-Shavit, Differential expression of protease activated receptor 1 (Par1) and pY397FAK in benign and malignant human ovarian tissue samples, Int. J. Cancer, 113 (2005) 372-378.

[25] D. Darmoul, V. Gratio, H. Devaud, T. Lehy, M. Laburthe, Aberrant expression and activation of the thrombin receptor protease-activated receptor-1 induces cell proliferation and motility in human colon cancer cells, Am. J. Pathol., 162 (2003) 1503-1513.

[26] P. Ghio, S. Cappia, G. Selvaggi, S. Novello, P. Lausi, G. Zecchina, M. Papotti, P. Borasio, G.V. Scagliotti, Prognostic role of protease-activated receptors 1 and 4 in resected stage IB nonsmall-cell lung cancer, Clin. Lung Cancer, 7 (2006) 395-400.

[27] X. Zhang, J.L. Hunt, D.P. Landsittel, S. Muller, K. Adler-Storthz, R.L. Ferris, D.M. Shin, Z.G. Chen, Correlation of protease-activated receptor-1 with differentiation markers in squamous cell carcinoma of the head and neck and its implication in lymph node metastasis, Clin. Cancer Res., 10 (2004) 8451-8459.

[28] C.S.B. Veiga, T.C. Carneiro-Lobo, C.J.B.P. Coelho, S.M.F. Carvalho, R.C. Maia, F.C. Vasconcelos, E. Abdelhay, A.L. Mencalha, A.F. Ferreira, F.A. Castro, R.Q. Monteiro, Increased expression of protease-activated receptor 1 (PAR-1) in human leukemias, Blood Cells Mol. Dis., 46 (2011) 230-234.

[29] N.E. Tsopanoglou, M.E. Maragoudakis, The role of thrombin in angiogenesis, in: M.E. Maragoudakis, N.E. Tsopanoglou (Eds.) Thrombin: Physiology and Disease, Springer, New York, 2009, pp. 93-113.

[30] S. Chackalamannil, Y. Xia, W.J. Greenlee, M. Clasby, D. Doller, H. Tsai, T. Asberom, M. Czarniecki, H.S. Ahn, G. Boykow, C. Foster, J. Agans-Fantuzzi, M. Bryant, J. Lau, M. Chintala, Discovery of potent orally active thrombin receptor (protease activated receptor 1) antagonists as novel antithrombotic agents, J. Med. Chem., 48 (2005) 5884-5887.

[31] S. Chackalamannil, Y. Wang, W.J. Greenlee, Z. Hu, Y. Xia, H.S. Ahn, G. Boykow, Y. Hsieh, J. Palamanda, J. Agans-Fantuzzi, S. Kurowski, M. Graziano, M. Chintala, Discovery of a novel, orally active himbacine-based thrombin receptor antagonist (SCH 530348) with potent antiplatelet activity, J. Med. Chem., 51 (2008) 3061-3064.

[32] Y. Shinohara, S. Goto, M. Doi, P. Jensen, Safety of the Novel Protease-Activated Receptor-1 Antagonist Vorapaxar in Japanese Patients with a History of Ischemic Stroke, J. Stroke Cerebrovasc. Dis., (2010) 10.1016/j.jstrokecerebrovasdis.2010.1009.1005.

[33] H.D. White, Oral antiplatelet therapy for atherothrombotic disease: Current evidence and new directions, Am. Heart J., 161 (2011) 450-461. 
[34] T.K. Vu, D.T. Hung, V.I. Wheaton, S.R. Coughlin, Molecular cloning of a functional thrombin receptor reveals a novel proteolytic mechanism of receptor activation, Cell, 64 (1991) 10571068.

[35] B.D. Blackhart, L. Ruslim-Litrus, C.C. Lu, V.L. Alves, W. Teng, R.M. Scarborough, E.E. Reynolds, D. Oksenberg, Extracellular mutations of protease-activated receptor-1 result in differential activation by thrombin and thrombin receptor agonist peptide, Mol. Pharmacol., 58 (2000) 1178-1187.

[36] T.K. Vu, V.I. Wheaton, D.T. Hung, I. Charo, S.R. Coughlin, Domains specifying thrombinreceptor interaction, Nature, 353 (1991) 674-677.

[37] Y.M. Ayala, A.M. Cantwell, T. Rose, L.A. Bush, D. Arosio, E. Di Cera, Molecular mapping of thrombin-receptor interactions, Proteins, 45 (2001) 107-116.

[38] M.T. Nieman, A.H. Schmaier, Interaction of thrombin with PAR1 and PAR4 at the thrombin cleavage site, Biochemistry, 46 (2007) 8603-8610.

[39] S.L. Jacques, M. LeMasurier, P.J. Sheridan, S.K. Seeley, A. Kuliopulos, Substrate-assisted catalysis of the PAR1 thrombin receptor. Enhancement of macromolecular association and cleavage, J. Biol. Chem., 275 (2000) 40671-44068.

[40] T. Myles, B.F. Le Bonniec, S.R. Stone, The dual role of thrombin's anion-binding exosite-I in the recognition and cleavage of the protease-activated receptor 1, Eur. J. Biochem., 268 (2001) 70-77.

[41] W.F. Bahou, J.L. Kutok, A. Wong, C.L. Potter, B.S. Coller, Identification of a novel thrombin receptor sequence required for activation-dependent responses, Blood, 84 (1994) 4195-4202.

[42] T. Nanevicz, M. Ishii, L. Wang, M. Chen, J. Chen, C.W. Turck, F.E. Cohen, S.R. Coughlin, Mechanisms of thrombin receptor agonist specificity. Chimeric receptors and complementary mutations identify an agonist recognition site, J. Biol. Chem., 270 (1995) 21619-21625.

[43] P.S. Gandhi, Z. Chen, F.S. Mathews, E. Di Cera, Structural identification of the pathway of long-range communication in an allosteric enzyme, Proc. Natl. Acad. Sci. U S A, 105 (2008) 1832-1837.

[44] P.S. Gandhi, Z. Chen, E. Di Cera, Crystal structure of thrombin bound to the uncleaved extracellular fragment of PAR1, J. Biol. Chem., 285 (2010) 15393-15398.

[45] S. Seeley, L. Covic, S.L. Jacques, J. Sudmeier, J.D. Baleja, A. Kuliopulos, Structural basis for thrombin activation of a protease-activated receptor: inhibition of intramolecular liganding, Chem. Biol., 10 (2003) 1033-1041.

[46] J.A. González-Vera, M.T. García-López, R. Herranz, Potential of amino acid-derived a-amino nitriles for generating molecular diversity, Mini-Rev. Org. Chem. , 5 (2008) 209-221.

[47] P. Ventosa-Andrés, M.T. García-López, R. Herranz, A study on the induction of stereoselectivity in the Strecker synthesis of basic amino acid-derived $\alpha$-amino nitriles, Tetrahedron: Asymmetry, (2012).doi: 10.1016/j.tetasy.2012.07.009.

[48] S. Herrero, M.T. García-López, R. Herranz, Expedient One-Pot Synthesis of Novel Chiral 2Substituted 5-Phenyl-1,4-benzodiazepine Scaffolds from Amino Acid-Derived Amino Nitriles, J. Org. Chem., 68 (2003) 4582-4585.

[49] P.J. Kocienski, Protecting Groups, 3rd ed., Georg Thieme, Stuttgart, 2004.

[50] Á.M. Valdivielso, M.T. García-López, R. Herranz, Improved synthesis of the PAR-1 thrombin receptor antagonist RWJ-58259, ARKIVOC, xvii (2008) 287-294.

[51] B.E. Maryanoff, H.C. Zhang, P. Andrade-Gordon, C.K. Derian, Discovery of potent peptidemimetic antagonists for the human thrombin receptor, protease-activated receptor-1 (PAR-1), Curr. Med. Chem. Cardiovasc. Hematol. Agents, 1 (2003) 13-36. 